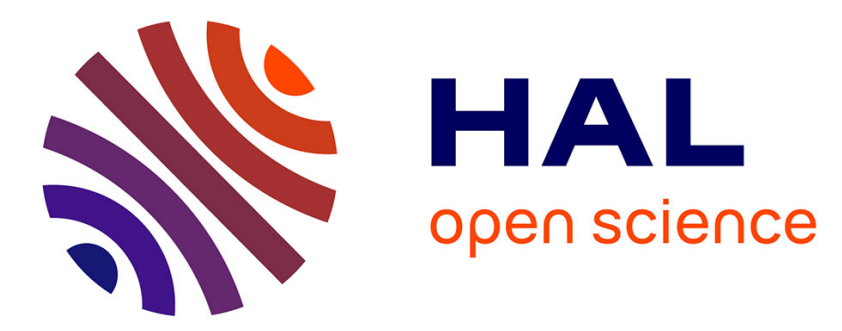

\title{
An entropy-stable Smooth Particle Hydrodynamics algorithm for large strain thermo-elasticity
}

Ataollah Ghavamian, Chun Hean Lee, Antonio J Gil, Javier Bonet, Thomas Heuzé, Laurent Stainier

\section{- To cite this version:}

Ataollah Ghavamian, Chun Hean Lee, Antonio J Gil, Javier Bonet, Thomas Heuzé, et al.. An entropystable Smooth Particle Hydrodynamics algorithm for large strain thermo-elasticity. Computer Methods in Applied Mechanics and Engineering, 2021, 379, pp.113736. 10.1016/j.cma.2021.113736 . hal03165085

\section{HAL Id: hal-03165085 \\ https://hal.science/hal-03165085}

Submitted on 10 Mar 2021

HAL is a multi-disciplinary open access archive for the deposit and dissemination of scientific research documents, whether they are published or not. The documents may come from teaching and research institutions in France or abroad, or from public or private research centers.
L'archive ouverte pluridisciplinaire HAL, est destinée au dépôt et à la diffusion de documents scientifiques de niveau recherche, publiés ou non, émanant des établissements d'enseignement et de recherche français ou étrangers, des laboratoires publics ou privés. 


\title{
An entropy-stable Smooth Particle Hydrodynamics algorithm for large strain thermo-elasticity
}

\author{
Ataollah Ghavamian ${ }^{a, 1}$, Chun Hean Lee ${ }^{b, 2}$, Antonio J. Gil ${ }^{a, 3}$, Javier Bonet ${ }^{c}$, Thomas \\ Heuzé $^{d}$, Laurent Stainier ${ }^{d}$ \\ (a) Zienkiewicz Centre for Computational Engineering, College of Engineering \\ Swansea University, Bay Campus, SA1 8EN, United Kingdom \\ (b) Glasgow Computational Engineering Centre, James Watt School of Engineering \\ University of Glasgow, Glasgow, G12 8QQ, United Kingdom \\ (c) University of Greenwich, London, SE10 9LS, United Kingdom \\ (d) Laboratory GeM, École Centrale de Nantes, 44300 Nantes, France
}

\begin{abstract}
This paper presents a novel Smooth Particle Hydrodynamics computational framework for the simulation of large strain fast solid dynamics in thermo-elasticity. The formulation is based on the Total Lagrangian description of a system of first order conservation laws written in terms of the linear momentum, the triplet of deformation measures (also known as minors of the deformation gradient tensor) and the total energy of the system, extending thus the previous work carried out by some of the authors in the context of isothermal elasticity and elasto-plasticity $[1-3]$. To ensure the stability (i.e. hyperbolicity) of the formulation from the continuum point of view, the internal energy density is expressed as a polyconvex combination of the triplet of deformation measures and the entropy density. Moreover, and to guarantee stability from the spatial discretisation point of view, consistently derived Riemann-based numerical dissipation is carefully introduced where local numerical entropy production is demonstrated via a novel technique in terms of the time rate of the so-called ballistic free energy of the system. For completeness, an alternative and equally competitive formulation (in the case of smooth solutions), expressed in terms of the entropy density, is also implemented and compared. A series of numerical examples is presented in order to assess the applicability and robustness of the proposed formulations, where the Smooth Particle Hydrodynamics scheme is benchmarked against an alternative in-house Finite Volume Vertex Centred implementation.
\end{abstract}

Keywords: Conservation laws, SPH, Upwind, Riemann Solver, Explicit dynamics, Thermo-elasticity

\section{Introduction}

Since the pioneering works of Lucy [4] and Monaghan [5], the field of Smooth Particle Hydrodynamics (SPH) has seen a dramatic development over the last few decades due to the attractiveness of the formulation, especially when dealing with very large deformation processes.

\footnotetext{
${ }^{1}$ Present address: École des Mines de Saint-Étienne, Saint-Étienne, France

${ }^{2}$ Corresponding author: chunhean.lee@glasgow.ac.uk

${ }^{3}$ Corresponding author: a.j.gil@swansea.ac.uk
} 
Numerous authors have steadily worked in the topic [6 20], fundamentally seeking the continuous enhancement of the original methodology. With focus on solid mechanics applications, the displacement-based formalism has been typically accepted as the preferred formulation and the state-of-art literature has focussed on addressing one or more of the following numerical deficiencies:

- existence of numerical instabilities of various types, such as the so-called tensile instability (when using a non-Lagrangian description of the problem) [6, 11, 21, 22], the appearance of zero-energy modes due to the rank-deficiency introduced as a result of using particle (reduced nodal) integration [10, 23], pressure spurious oscillations in the vicinity of near incompressibility [1, 24, 25] and the possible development of long term instabilities [12], and

- development of numerical errors associated to lack of conservation, consistency and reduced order of convergence for derived variables [26 28]

Some of these numerical problems have been partially alleviated through the use of corrected kernel gradient approximations [6, 7] combined with so-called non-consistent stabilisation strategies [5]. Interestingly, another recently developed mesh-free method, namely the Peridynamics state-based approach [29 32], has been shown [9] to be equivalent to a Total Lagrangian SPH scheme, when used in conjunction with corrected derivatives and nodal integration (or collocation). This equivalence implies that most of the numerical shortcomings described above for SPH schemes are persistent in peridynamics based methods. As a result, and despite the tremendous development in the field, the ab initio stability of SPH schemes is still an open problem.

In a recent series of papers in the context of isothermal hyperelasticity and elastoplasticity [1-3], some of the authors of the present manuscript have successfully introduced a mixed based SPH computational framework for explicit fast solid isothermal dynamics, where the conservation of linear momentum $\boldsymbol{p}$ is solved along with geometric conservation equations for the deformation gradient tensor $\boldsymbol{F}$, the co-factor of the deformation gradient tensor $\boldsymbol{H}$ and the Jacobian of the deformation gradient tensor $J$. In addition to ensure conservation and equal (second) order of convergence for all derived variables, the short and long term stability of the proposed schemes is achieved through a variety of alternative, and consistently derived, numerical schemes inspired from the field of Computational Fluid Dynamics, namely, Jameson-Schmidt-Turkel [1], Streamline-Upwind Petrov-Galerkin [2] and Upwinding [3. In all these schemes, tailor-made numerical dissipation is added with the purpose of addressing the instabilities inherently due to the use of nodal (particle) integration, without jeopardising either the conservation or the numerical accuracy of the scheme.

Consideration of thermal effects, especially in the context of large strain fast transient dynamics, is fundamental in order to obtain a realistic representation of stresses when a solid undergoes a complex and rapidly evolving thermally-coupled deformation pattern [33 41]. With focus on thermo-elasticity and thermo-inelasticity, numerous authors have worked over the years putting forward a variety of computational schemes where both displacements and thermal variables are solved either monolithically or in staggered fashion [42 47]. Traditionally, authors prefer the use of the temperature $\theta$ as the thermal unknown to be solved instead of the entropy density $\eta$ (although the latter is equally plausible [42, 48]).

The aim of this paper is to further extend the work thus far presented in the context of isothermal elasticity [1 3] to account for possible strongly thermally-coupled scenarios, through 
the consideration of thermo-elastic constitutive model 4 . As a result, a generalised system of first order conservation laws is presented where the first law of thermodynamics, written in terms of the total energy of the system $E$, is also solved in addition to the conservation of linear momentum and the geometric conservation laws. Furthermore, and in the case of smooth solutions, the resulting $\{\boldsymbol{p}, \boldsymbol{F}, \boldsymbol{H}, J, E\}$ formulation will be compared against an alternative, albeit equally competitive, $\{\boldsymbol{p}, \boldsymbol{F}, \boldsymbol{H}, J, \eta\}$ formulation.

A crucial aspect that requires special attention (especially in the case of coupled problems) is that of the stability of the formulation, from both the continuum and numerical standpoints. With respect to the former, this paper seeks the use of ab initio stable polyconvex constitutive internal energy functionals in the most generic thermal case [49], that is, not only restricted to isothermal or isentropic scenarios. With that in mind, internal energy density functionals which are (objectively) polyconvex in the extended set of arguments $\{\boldsymbol{F}, \boldsymbol{H}, J, \eta\}$ are exploited in this work [50], ensuring existence of solutions regardless of the level of strains and thermal fields. As for the spatial discretisation, a Riemann based (upwinding) approach is pursued where a consistently derived numerical stabilisation is introduced ensuring local production of numerical entropy. The latter is demonstrated by the monitoring of the so-called ballistic energy of the system [51].

The paper is organised as follows. Section 2 starts by presenting the complete set of conservation laws which will be solved, encompassing linear momentum, geometric conservation laws and first law of thermodynamics. This is followed by the introduction of appropriate $a b$ initio stable polyconvex strain energy density functionals for two prototypical thermo-elastic models, namely, modified entropic elasticity and Mie-Grüneisen. Section 3 presents the variational statements of the problem as well the second law of thermodynamics written in terms of the so-called ballistic free energy. Section 4 presents the Smooth Particle Hydrodynamics discretisation numerical scheme where special attention is paid to the Riemann based (upwinding) numerical dissipation employed. A proof of entropy production is included as a necessary condition for stability at the semi-discrete level. Section 5 briefly presents the two-stage explicit Runge-Kutta scheme used to evolve the semi-discrete equations in time. Section 6 simply includes the algorithmic flowchart of the resulting numerical scheme. Section 7 presents a wide spectrum of numerical examples in order to assess the convergence, conservation and stability of the computational framework, where comparisons will be carried out against an alternative Vertex Centred Finite Volume implementation. Section 8 presents some concluding remarks. In addition, the paper includes an appendix summarising key aspects related to the computation of the entropy conjugates of the conservation variables with respect to the ballistic energy.

\section{Irreversible elastodynamics}

Consider the motion of a thermo-elastic body that is described by a time-dependent mapping field $\phi(\boldsymbol{X}, t)$ which links a material particle from initial reference configuration $\boldsymbol{X} \in V_{R}$ of boundary $\partial V_{R}$ (with outward unit normal $\boldsymbol{N}$ ) to the time-dependent spatial configuration $\boldsymbol{x} \in V_{R}(t)$ of boundary $\partial V_{R}(t)$ (with outward unit normal $\boldsymbol{n}$ ) according to $\boldsymbol{x}=\boldsymbol{\phi}(\boldsymbol{X}, t)$. In addition, the elastic body can experience thermal variations described by the evolution of the temperature field $\theta(\boldsymbol{X}, t)$. In general, the thermo-mechanical evolution of the body can be

\footnotetext{
${ }^{4}$ Further inelastic contributions (i.e. plasticity, viscoplasticity, viscoelasticity, thermo-viscoplasticity) are not within the scope of this paper.
} 
described [50] by the following system of first order Total Lagrangian conservation laws

$$
\begin{aligned}
& \frac{\partial \boldsymbol{p}}{\partial t}-\operatorname{DIV} \boldsymbol{P}=\boldsymbol{f}_{R} ; \\
& \frac{\partial \boldsymbol{F}}{\partial t}-\operatorname{DIV}(\boldsymbol{v} \otimes \boldsymbol{I})=\mathbf{0} \\
& \frac{\partial \boldsymbol{H}}{\partial t}-\operatorname{CURL}(\boldsymbol{v} \times \boldsymbol{F})=\mathbf{0} \\
& \frac{\partial J}{\partial t}-\operatorname{DIV}\left(\boldsymbol{H}^{T} \boldsymbol{v}\right)=0 ; \\
& \frac{\partial E}{\partial t}-\operatorname{DIV}\left(\boldsymbol{P}^{T} \boldsymbol{v}-\boldsymbol{Q}\right)=\boldsymbol{f}_{R} \cdot \boldsymbol{v}+s_{R} .
\end{aligned}
$$

In above system of equations, $\boldsymbol{p}=\rho_{R} \boldsymbol{v}$ is the linear momentum per unit undeformed volume, $\rho_{R}$ is the density of the undeformed configuration, $\boldsymbol{v}$ represents the velocity field, $\boldsymbol{f}_{R}$ is the body force per unit undeformed volume, $\boldsymbol{F}$ is the deformation gradient tensor (or fibre map), $\boldsymbol{H}$ is the co-factor of the deformation gradient tensor (or area map), $J$ is the Jacobian of the deformation gradient tensor (or volume map), $E$ is the total energy per unit undeformed volume, $\boldsymbol{P}$ represents the first Piola-Kirchhoff stress tensor, $\boldsymbol{Q}$ represents the so-called material or Lagrangian heat flux vector, $s_{R}$ denotes a possible heat source term and DIV and CURL represent the material divergence and curl operators, respectively, carried out with respect to undeformed configuration. The symbol $\times$ represents the tensor cross product between vectors and/or second order tensors, originally introduced in [52, 53] and used for the first time in the context of continuum mechanics in [54, 55].

Equation (1a) represents the conservation of linear momentum. Equations (1b) to (1d) represent geometric conservation laws for the triplet of deformation measures $\{\boldsymbol{F}, \boldsymbol{H}, J\}^{5}$. Finally, equation (1e) represents the first law of thermodynamics written in terms of the total energy $E$. As the system of conservation laws presented above has more equations than needed, suitable compatibility relationships, also known as involutions [56, 57], are necessary, namely

$$
\mathrm{CURL} \boldsymbol{F}=\mathbf{0} ; \quad \text { DIV } \boldsymbol{H}=\mathbf{0} .
$$

These two equations allow the geometric conservation laws $(1 \mathrm{c})$ and $(1 \mathrm{~d})$ to be written in an alternative non-conservative form as

$$
\begin{gathered}
\frac{\partial \boldsymbol{H}}{\partial t}=\boldsymbol{F} \times \nabla_{0} \boldsymbol{v} \\
\frac{\partial J}{\partial t}=\boldsymbol{H}: \nabla_{0} \boldsymbol{v} .
\end{gathered}
$$

It is now possible to combine all of the Total Lagrangian conservation equations described in (1) into a single system of conservation equations 6

$$
\frac{\partial \mathcal{U}}{\partial t}+\sum_{I=1}^{3} \frac{\partial \mathcal{F}_{I}}{\partial X_{I}}=\mathcal{S}
$$

\footnotetext{
${ }^{5}$ The definitions for the triplet of deformation measures are $\boldsymbol{F}=\nabla_{0} \phi, \boldsymbol{H}=\frac{1}{2} \boldsymbol{F} \times \boldsymbol{F}$ and $J=\frac{1}{3} \boldsymbol{H}: \boldsymbol{F}$, respectively, [54, 55].

${ }^{6}$ In the presence of non-smooth solutions, above balance equations (1) are accompanied by appropriate Rankine Hugoniot jump conditions in the reference space [1 $1+3,58,63$.
} 
where $\mathcal{U}$ is the vector of conservation variables, $\mathcal{F}_{I}$ is the flux column vector in $I$-th material direction and $\mathcal{S}$ is the source term, which can be written as

$$
\mathcal{U}=\left[\begin{array}{c}
\boldsymbol{p} \\
\boldsymbol{F} \\
\boldsymbol{H} \\
J \\
E
\end{array}\right], \quad \mathcal{F}_{I}=-\left[\begin{array}{c}
\boldsymbol{P} \boldsymbol{E}_{I} \\
\boldsymbol{v} \otimes \boldsymbol{E}_{I} \\
\boldsymbol{F} \times\left(\boldsymbol{v} \otimes \boldsymbol{E}_{I}\right) \\
\boldsymbol{H}:\left(\boldsymbol{v} \otimes \boldsymbol{E}_{I}\right) \\
\boldsymbol{v} \cdot \boldsymbol{P} \boldsymbol{E}_{I}-\boldsymbol{Q} \cdot \boldsymbol{E}_{I}
\end{array}\right], \quad \mathcal{S}=\left[\begin{array}{c}
\boldsymbol{f}_{R} \\
\mathbf{0} \\
\mathbf{0} \\
0 \\
\boldsymbol{f}_{R} \cdot \boldsymbol{v}+s_{R}
\end{array}\right]
$$

and the Cartesian coordinate basis

$$
\boldsymbol{E}_{1}=\left[\begin{array}{c}
1 \\
0 \\
0
\end{array}\right] ; \quad \boldsymbol{E}_{2}=\left[\begin{array}{c}
0 \\
1 \\
0
\end{array}\right] ; \quad \boldsymbol{E}_{3}=\left[\begin{array}{l}
0 \\
0 \\
1
\end{array}\right]
$$

For closure of system (1), suitable relationships between the heat flux vector $\boldsymbol{Q}$ and the first Piola-Kirchhoff stress tensor $\boldsymbol{P}$ must be given in terms of the conservation variables $\mathcal{U}$. Specifically, a conductive type heat flux vector will be adopted in this paper and presented in the following section. Moreover, in order to guarantee the existence of real wave speeds in the material (also known as hyperbolicity) for the entire range of possible deformations and thermal variations, suitable constitutive laws compliant with the principle of objectivity (frame invariance) and the second law of Thermodynamics (Coleman-Noll procedure) will be discussed in the following section. Finally, for a complete definition of the initial boundary value problem, initial and boundary (essential and natural) conditions must also be specified as appropriate.

\subsection{Constitutive relationships for stresses and heat flux}

The total energy $E$ in the above first law of thermodynamics $1 \mathrm{e}$ includes kinetic and internal energy components. Multiplying the linear momentum conservation equation (1a) by $\boldsymbol{v}$ and subtracting it from equation (1e), after some algebra?, gives [50]

$$
\frac{\partial \mathscr{E}}{\partial t}+\operatorname{DIV} \boldsymbol{Q}=\boldsymbol{P}: \nabla_{0} \boldsymbol{v}+s_{R}
$$

where $\mathscr{E}(\boldsymbol{X}, t)=E-\frac{1}{2 \rho_{R}}(\boldsymbol{p} \cdot \boldsymbol{p})$ represents the internal energy per unit undeformed volume. In the current paper, we consider the case of strict thermo-elasticity, where the internal energy $\mathscr{E}(\boldsymbol{X}, t)$ is postulated to depend on the triplet of deformation measures $\{\boldsymbol{F}, \boldsymbol{H}, J\}$ and the entropy density per unit undeformed volume $\eta(\boldsymbol{X}, t)$, as

$$
\mathscr{E}(\boldsymbol{X}, t)=\mathcal{E}\left(\mathcal{X}_{\eta}\right) ; \quad \mathcal{X}_{\eta}=\{\mathcal{X}, \eta\} ; \quad \mathcal{X}=\{\boldsymbol{F}, \boldsymbol{H}, J\}
$$

where $\mathscr{E}$ and $\mathcal{E}$ denote two alternative functional representations of the same magnitude. As it is customary[64], entropy $\eta$ and temperature $\theta$ are defined as conjugates of each other with respect to the internal energy density $\mathscr{E}$, namely

$$
\theta(\boldsymbol{X}, t)=\frac{\partial \mathcal{E}\left(\boldsymbol{\mathcal { X }}_{\eta}\right)}{\partial \eta}=\Theta\left(\boldsymbol{\mathcal { X }}_{\eta}\right)
$$

where $\theta$ and $\Theta$ are used to denote the same temperature magnitude albeit with different functional dependency. It is clear that the coupling between the thermal and the deformation

\footnotetext{
${ }^{7}$ This transformation is only possible in the case of smooth fields.
} 
process takes place via the dependency of the temperature on the triplet $\mathcal{X}$ within $\boldsymbol{\mathcal { X }}_{\eta}$. Similarly, energy conjugate fields can be defined to the three deformation measures of the triplet $\mathcal{X}$ as [50]

$$
\boldsymbol{\Sigma}_{\boldsymbol{F}}\left(\boldsymbol{\mathcal { X }}_{\eta}\right)=\frac{\partial \mathcal{E}\left(\boldsymbol{\mathcal { X }}_{\eta}\right)}{\partial \boldsymbol{F}} ; \quad \boldsymbol{\Sigma}_{\boldsymbol{H}}\left(\boldsymbol{\mathcal { X }}_{\eta}\right)=\frac{\partial \mathcal{E}\left(\boldsymbol{\mathcal { X }}_{\eta}\right)}{\partial \boldsymbol{H}} ; \quad \Sigma_{J}\left(\boldsymbol{\mathcal { X }}_{\eta}\right)=\frac{\partial \mathcal{E}\left(\boldsymbol{\mathcal { X }}_{\eta}\right)}{\partial J}
$$

Coupling between the thermal and the deformation process takes place via the dependency of the conjugate stresses on the entropy $\eta$ within $\mathcal{X}_{\eta}$. With (9) and (10) at hand, it is now possible to relate the conjugate stresses defined in (10) to the standard first Piola-Kirchhoff stress tensor. This can be achieved by comparing the time rate of the internal energy density $\mathscr{E}(\boldsymbol{X}, t)$ (7) to that of its equivalent re-expression $\mathcal{E}\left(\boldsymbol{\mathcal { X }}_{\eta}\right)$ (8) via the chain rule, and using the properties of the tensor cross product as shown in [58], to give

$$
\begin{aligned}
\frac{\partial \mathscr{E}(\boldsymbol{X}, t)}{\partial t} & =\frac{\partial \mathcal{E}\left(\boldsymbol{\mathcal { X }}_{\eta}\right)}{\partial \boldsymbol{F}}: \frac{\partial \boldsymbol{F}}{\partial t}+\frac{\partial \mathcal{E}\left(\boldsymbol{\mathcal { X }}_{\eta}\right)}{\partial \boldsymbol{H}}: \frac{\partial \boldsymbol{H}}{\partial t}+\frac{\partial \mathcal{E}\left(\boldsymbol{\mathcal { X }}_{\eta}\right)}{\partial J} \frac{\partial J}{\partial t}+\frac{\partial \mathcal{E}\left(\boldsymbol{\mathcal { X }}_{\eta}\right)}{\partial \eta} \frac{\partial \eta}{\partial t} \\
& =\boldsymbol{\Sigma}_{\boldsymbol{F}}: \boldsymbol{\nabla}_{0} \boldsymbol{v}+\boldsymbol{\Sigma}_{\boldsymbol{H}}:\left(\boldsymbol{F} \times \boldsymbol{\nabla}_{0} \boldsymbol{v}\right)+\Sigma_{J}\left(\boldsymbol{H}: \boldsymbol{\nabla}_{0} \boldsymbol{v}\right)+\theta \frac{\partial \eta}{\partial t} \\
& =\left[\boldsymbol{\Sigma}_{\boldsymbol{F}}+\boldsymbol{\Sigma}_{\boldsymbol{H}} \times \boldsymbol{F}+\Sigma_{J} \boldsymbol{H}\right]: \boldsymbol{\nabla}_{0} \boldsymbol{v}+\theta \frac{\partial \eta}{\partial t}
\end{aligned}
$$

which leads to the following relationship

$$
\boldsymbol{P}\left(\mathcal{X}_{\eta}\right)=\Sigma_{\boldsymbol{F}}+\Sigma_{\boldsymbol{H}} \times \boldsymbol{F}+\Sigma_{J} \boldsymbol{H}
$$

Concerning the heat flux vector $\boldsymbol{Q}$, we consider the typical Fourier law to hold ${ }^{8}$ and which can be defined in a Total Lagrangian fashion as

$$
\boldsymbol{Q}=-\boldsymbol{K} \nabla_{0} \theta ; \quad \boldsymbol{K}=J^{-1} \boldsymbol{H}^{T} \boldsymbol{k} \boldsymbol{H},
$$

where $\boldsymbol{k}$ represents the positive semi-definite second order thermal conductivity tensor in the deformed configuration. For the case of isotropy, above thermal conductivity tensor can then be expressed in terms of the scalar conductivity field $h$, that is $\boldsymbol{k}=h \boldsymbol{I}$.

Notice that in expressions (12) and (13), the constitutive relationships derived for both the first Piola-Kirchhoff stress tensor and the heat flux are expressed as functions of the triplet of deformations $\mathcal{X}$ and either the entropy $\eta$ or the temperature $\theta$. The remaining aspect to be addressed is how to relate the thermal unknown of the system, that is the total energy $E$ in this case, with entropy $\eta$ and temperature $\theta$. This will be discussed in the following section.

Remark 1: In this paper we also explore an alternative formulation by re-writing the first law of thermodynamics (1e) in terms of the entropy density $\eta(\boldsymbol{X}, t)$. Proceeding in this manner, and in the case of strict thermo-elasticity, would give a thermal expression in which mechanical terms can be conveniently eliminated to yield [50]

$$
\theta \frac{\partial \eta}{\partial t}+\operatorname{DIV} \boldsymbol{Q}=s_{R}
$$

\footnotetext{
${ }^{8}$ Technically speaking, this term transforms the equations into parabolic. However, for sufficiently small values of this term, the formulation can be regarded as fundamentally hyperbolic.
} 
Alternatively, noting that $\frac{1}{\theta} \operatorname{DIV} \boldsymbol{Q}=\operatorname{DIV}\left(\frac{\boldsymbol{Q}}{\theta}\right)+\frac{1}{\theta^{2}} \boldsymbol{Q} \cdot \boldsymbol{\nabla}_{0} \theta$, a conservation type of law for the entropy emerges as

$$
\frac{\partial \eta}{\partial t}+\operatorname{DIV}\left(\frac{\boldsymbol{Q}}{\theta}\right)=\frac{s_{R}}{\theta}-\frac{1}{\theta^{2}} \boldsymbol{Q} \cdot \boldsymbol{\nabla}_{0} \theta,
$$

where $\boldsymbol{Q} / \theta$ represents the flux of entropy and terms on the right hand side are collectively known as the entropy source per unit undeformed volume. Note that this expression for the entropy assumes smooth solutions and it is still an expression of the first law. Combination of equation (15) with the linear momentum equation $\boldsymbol{p}(1 \mathrm{a})$ and the geometric conservation laws for $\{\boldsymbol{F}, \boldsymbol{H}, J\}(1 \mathrm{~b})-1 \mathrm{~d})$ yields an alternative set of conservation laws in terms of $\{\boldsymbol{p}, \boldsymbol{F}, \boldsymbol{H}, J, \eta\}$, in contrast to the set $\{\boldsymbol{p}, \boldsymbol{F}, \boldsymbol{H}, J, E\}$ featuring in (5).

\subsection{General thermal relationships}

In general, the Calorimetry relationships between internal energy $\mathcal{E}$, temperature $\theta$ and entropy $\eta$ can be derived from the definition of the specific heat at constant volume $c_{v}[50$ ]. This requires the re-definition of the entropy $\eta(\boldsymbol{X}, t)$ and the internal energy density $\mathscr{E}(\boldsymbol{X}, t)$ in terms of the triplet of deformation measures $\mathcal{X}$ and the temperature $\theta$ of the system, namely, $\mathcal{X}_{\theta}=\{\boldsymbol{X}, \theta\}=\{\boldsymbol{F}, \boldsymbol{H}, J, \theta\}$. Specifically,

$$
\left.c_{v} \stackrel{\text { def }}{=} \frac{d \mathscr{E}}{d \theta}\right|_{\mathcal{X}=\text { const }}=\frac{\partial \tilde{\mathcal{E}}\left(\boldsymbol{\mathcal { X }}_{\theta}\right)}{\partial \theta} ; \quad \tilde{\mathcal{E}}\left(\boldsymbol{\mathcal { X }}_{\theta}\right)=\mathcal{E}\left(\mathcal{X}, \tilde{\eta}\left(\boldsymbol{\mathcal { X }}_{\theta}\right)\right) ; \quad \eta(\boldsymbol{X}, t)=\tilde{\eta}\left(\boldsymbol{\mathcal { X }}_{\theta}\right),
$$

with $c_{v}=\rho_{R} C_{v}>0$, where $\rho_{R}$ is the density measured at a reference temperature $\theta_{R}$ and $C_{v}$ the specific heat per unit mass.

As the internal energy $\mathscr{E}(\boldsymbol{X}, t)$ can be expressed as a function of the set of arguments $\mathcal{X}_{\eta}$ (8), and given the fact that $\partial \mathcal{E}\left(\mathcal{X}_{\eta}\right) / \partial \eta=\theta(9)$, a constitutive relationship between the temperature $\theta$ and the entropy $\eta$ at constant deformation can be established [50] by recasting equation (16) using the chain rule to yield

$$
\frac{\partial \tilde{\eta}\left(\boldsymbol{\mathcal { X }}_{\theta}\right)}{\partial \theta}=\frac{c_{v}}{\theta} .
$$

Restricting the derivation to the simple constant heat coefficient case, enables expression (17) to be integrated analytically with respect to the entropy or temperature changes as

$$
\int_{\tilde{\eta}_{R}(\mathcal{X})}^{\tilde{\eta}\left(\mathcal{X}_{\theta}\right)} d \eta=c_{v} \int_{\theta_{R}}^{\theta} \frac{1}{\theta} d \theta,
$$

which leads to a simple relationship between entropy and temperature as

$$
\tilde{\eta}\left(\mathcal{X}_{\theta}\right)=\tilde{\eta}_{R}(\mathcal{X})+c_{v} \ln \frac{\theta}{\theta_{R}} ; \quad \tilde{\eta}_{R}(\mathcal{X})=\tilde{\eta}\left(\mathcal{X}, \theta=\theta_{R}\right)
$$

In this expression, $\tilde{\eta}_{R}(\boldsymbol{\mathcal { X }})$ denotes the entropy measured at a constant (reference) temperature $\theta_{R}$ and expressed as a function of the deformation $\mathcal{X}$ (i.e. after the deformation, the temperature is allowed to return to the reference value $\theta_{R}$ ).

Using expression (19), and noting the alternative functional representation $\eta(\boldsymbol{X}, t)=\tilde{\eta}\left(\boldsymbol{\mathcal { X }}_{\theta}\right)$ (16), the reverse relationship yielding the temperature as a function of the deformation and entropy is given by

$$
\Theta\left(\mathcal{X}_{\eta}\right)=\theta_{R} e^{\left(\eta-\tilde{\eta}_{R}(\mathcal{X})\right) / c_{v}}
$$


Again, with the assumption of a constant specific heat coefficient $c_{v}$, it is possible to write an explicit relationship for the internal energy $\mathscr{E}(\boldsymbol{X}, t)$ as functions of deformations $\mathcal{X}$ and temperature $\theta$, that is $\tilde{\mathcal{E}}\left(\boldsymbol{\mathcal { X }}_{\theta}\right)$, instead of $\mathcal{E}\left(\boldsymbol{\mathcal { X }}_{\eta}\right)$. This is achieved by directly integrating equation (16) with respect to temperature between the limits $\theta_{R}$ and a given value $\theta$ to give

$$
\int_{\tilde{\mathcal{E}}_{R}(\boldsymbol{\mathcal { X }})}^{\tilde{\mathcal{E}}\left(\boldsymbol{\mathcal { X }}_{\theta}\right)} d \mathcal{E}=c_{v} \int_{\theta_{R}}^{\theta} d \theta
$$

which then yields

$$
\tilde{\mathcal{E}}\left(\mathcal{X}_{\theta}\right)=\tilde{\mathcal{E}}_{R}(\mathcal{X})+c_{v} \vartheta ; \quad \tilde{\mathcal{E}}_{R}(\mathcal{X})=\tilde{\mathcal{E}}\left(\mathcal{X}, \theta=\theta_{R}\right) ; \quad \vartheta=\theta-\theta_{R} .
$$

Here, $\vartheta$ represents the temperature change (with respect the reference temperature $\theta_{R}$ ) and the term $\tilde{\mathcal{E}}_{R}(\mathcal{X})$ represents the internal energy per unit reference volume caused by the deformation after the temperature has been allowed to return back to the reference value $\theta_{R}$.

Since the terms $\mathscr{E}(\boldsymbol{X}, t)$ and $\tilde{\mathcal{E}}\left(\boldsymbol{\mathcal { X }}_{\theta}\right)$ are used to denote the same energy (with different functional dependency), and recalling that $\mathscr{E}(\boldsymbol{X}, t)=E-\frac{1}{2 \rho_{R}}(\boldsymbol{p} \cdot \boldsymbol{p})$, rearrangement of equation (22) gives the temperature update as

$$
\begin{aligned}
\hat{\theta}\left(\boldsymbol{p}, \boldsymbol{\mathcal { X }}_{E}\right) & =\theta_{R}+\frac{1}{c_{v}}\left(\tilde{\mathcal{E}}\left(\boldsymbol{\mathcal { X }}_{\theta}\right)-\tilde{\mathcal{E}}_{R}(\boldsymbol{\mathcal { X }})\right) \\
& =\theta_{R}+\frac{1}{c_{v}}\left(E-\frac{1}{2 \rho_{R}} \boldsymbol{p} \cdot \boldsymbol{p}-\tilde{\mathcal{E}}_{R}(\boldsymbol{\mathcal { X }})\right),
\end{aligned}
$$

where $\boldsymbol{\mathcal { X }}_{E}=\{\mathcal{X}, E\}$. Equation (23) provides an expression of the temperature in terms of the linear momentum, the triplet of deformation measures and the total energy density, namely, $\theta(\boldsymbol{X}, t)=\hat{\theta}\left(\boldsymbol{p}, \boldsymbol{\mathcal { X }}_{E}\right)$. Moreover, noticing equation $(19)$, it is also possible to obtain a similar relationship between the entropy density $\eta$ and the set $\boldsymbol{\mathcal { X }}_{E}$, namely, $\eta(\boldsymbol{X}, t)=\hat{\eta}\left(\boldsymbol{p}, \boldsymbol{\mathcal { X }}_{E}\right)$, that is

$$
\hat{\eta}\left(\boldsymbol{p}, \boldsymbol{\mathcal { X }}_{E}\right)=\tilde{\eta}_{R}(\boldsymbol{\mathcal { X }})+c_{v} \ln \left(\frac{\hat{\theta}\left(\boldsymbol{p}, \boldsymbol{\mathcal { X }}_{E}\right)}{\theta_{R}}\right)
$$

A final useful relationship necessary to compute the conjugate stresses $\left\{\boldsymbol{\Sigma}_{\boldsymbol{F}}, \boldsymbol{\Sigma}_{\boldsymbol{H}}, \Sigma_{J}\right\}$ 10 is that of the internal energy density, namely

$$
\hat{\mathcal{E}}\left(\boldsymbol{p}, \mathcal{X}_{\boldsymbol{E}}\right)=E-\frac{1}{2 \rho_{R}} \boldsymbol{p} \cdot \boldsymbol{p},
$$

where $\hat{\mathcal{E}}\left(\boldsymbol{p}, \boldsymbol{\mathcal { X }}_{E}\right)$ represents an alternative functional representation of the internal energy $\mathcal{E}\left(\boldsymbol{\mathcal { X }}_{\eta}\right)$. Finally, in order to complete the definition of the thermo-elastic constitutive model (refer to equations (23), (24) and (25)), it is necessary to provide suitable functional expressions for $\tilde{\mathcal{E}}_{R}(\mathcal{X})$ and $\tilde{\eta}_{R}(\mathcal{\mathcal { X }})$, which will depend on the specific material under consideration.

Following [50], in order to guarantee the existence of real wave speeds (hyperbolicity) in the material for the entire thermo-elastic deformation process, that is regardless of the amount of deformation $\mathcal{X}$ and thermal state $\eta$ (or $\theta$ ), a sufficient condition is that of selecting $\tilde{\mathcal{E}}_{R}(\boldsymbol{\mathcal { X }})$ and $-\tilde{\eta}_{R}(\boldsymbol{\mathcal { X }})$ to be convex in $\mathcal{X}$, that is both functions shall be polyconvex. For further details, the reader is referred to [50]. In this work, two well-established thermo-elastic models derived from universally polyconvex strain energy functions will be used, namely modified entropic elasticity and Mie-Grüneisen. The reader is referred to [50] where hyperbolicity is demonstrated for these two thermo-elastic constitutive models where the closed-form computation of bounds for the propagating wave speeds is also shown. 


\subsubsection{Modified Entropic Elasticity (MEE)}

The internal energy density at reference temperature $\theta_{R}$ for this material is typically given by a purely volumetric function as 50 ]

$$
\tilde{\mathcal{E}}_{R}^{M E E}(J)=c_{v} \Gamma_{0} \theta_{R}(J-1),
$$

where $\Gamma_{0}$ is a positive material constant. In addition, the entropy function at reference temperature $\theta_{R}$ is given by

$$
\tilde{\eta}_{R}^{M E E}(\mathcal{X})=\frac{1}{\theta_{R}}\left(\tilde{\mathcal{E}}_{R}^{M E E}(J)-\psi_{R}^{M E E}(\mathcal{X})\right)
$$

where $\psi_{R}^{M E E}(\boldsymbol{\mathcal { X }})$ is the Helmholtz's free energy function at reference temperature $\theta_{R}$, where a possible deviatoric-volumetric expression of the Mooney-Rivlin type is

$$
\psi_{R}^{M E E}(\boldsymbol{\mathcal { X }})=\varsigma_{R}\left(J^{-2 / 3}(\boldsymbol{F}: \boldsymbol{F})-3\right)+\xi_{R}\left(J^{-2}(\boldsymbol{H}: \boldsymbol{H})^{3 / 2}-3 \sqrt{3}\right)+\frac{\kappa_{R}}{2}(J-1)^{2},
$$

where $\left\{\varsigma_{R}, \xi_{R}, \kappa_{R}\right\}$ are material parameters. Notice that if $\xi_{R}=0$, the material degenerates to a neo-Hookean type of model. Typically, these material parameters and $\Gamma_{0}$ introduced above, are calibrated against those of linear elasticity, namely, shear modulus $\mu$, bulk modulus $\kappa$ and thermal expansion coefficient $\alpha$ as 65 ]

$$
\mu=2 \varsigma_{R}+3 \sqrt{3} \xi_{R} ; \quad \kappa=\kappa_{R} ; \quad \alpha=\frac{c_{v} \Gamma_{0}}{3 \kappa} .
$$

\subsubsection{Mie-Grüneisen ( $M G)$}

A plausible deviatoric-volumetric expression for the internal energy density of the MooneyRivlin type at reference temperature $\theta_{R}$ for this material can be given as

$$
\tilde{\mathcal{E}}_{R}^{M G}(\boldsymbol{\mathcal { X }})=\zeta_{R}\left(J^{-2 / 3}(\boldsymbol{F}: \boldsymbol{F})-3\right)+\gamma_{R}\left(J^{-2}(\boldsymbol{H}: \boldsymbol{H})^{3 / 2}-3 \sqrt{3}\right)+\frac{\chi_{R}}{2}(J-1)^{2}+c_{v} \theta_{R} \Gamma_{0}(J-1),
$$

where $\left\{\zeta_{R}, \gamma_{R}, \chi_{R}\right\}$ are material parameters (if $\gamma_{R}=0$, the material degenerates to a neoHookean type of model) and the entropy function at reference temperature $\theta_{R}$ is given by

$$
\tilde{\eta}_{R}^{M G}(\mathcal{X})=c_{v} \Gamma_{0}\left(\frac{J^{q}-1}{q}\right)
$$

where $q$ is a dimensionless coefficient that varies from zero (for a perfect gas) to one (for solid materials). The material parameters can be calibrated against those of linear elasticity, namely, shear modulus $\mu$, bulk modulus $\kappa$ and thermal expansion coefficient $\alpha$ as

$$
\mu=2 \zeta_{R}+3 \sqrt{3} \gamma_{R} ; \quad \kappa=\chi_{R}+c_{v} \theta_{R} \Gamma_{0}(1-q) ; \quad \alpha=\frac{c_{v} \Gamma_{0}}{3 \kappa} .
$$

\section{Weak form statements}

In general, a standard weak variational statement for the thermo-mechanical system 1a 1e is established by multiplying the local differential equations (4) (written in terms of the conservation variables $\mathcal{U}$ ) with their appropriate work conjugate virtual fields $\delta \mathcal{V}$, and integrating over the reference domain $V_{R}$ of the body, to give

$$
\int_{V_{R}} \delta \mathcal{V} \bullet \frac{\partial \mathcal{U}}{\partial t} d V_{R}-\int_{V_{R}} \delta \mathcal{V} \bullet \mathcal{S} d V_{R}+\int_{V_{R}} \delta \mathcal{V} \bullet \frac{\partial \mathcal{F}_{I}}{\partial X_{I}} d V_{R}=0
$$


where the symbol $\bullet$ is used to denote the inner (dual) product of work conjugate pairs.

In order to give a proper physical meaning to the conjugate virtual fields $\delta \mathcal{V}$ and pave the way for the proof of numerical entropy production presented in a subsequent section, it is important to introduce the concept of ballistic free energy $B=B(\boldsymbol{X}, t)$ [51]. As already shown in [50], the ballistic free energy can be used as the thermodynamic convex entropy function ${ }^{9}$ of the system of conservation laws (4), from where so-called entropy conjugate fields ${ }^{10} \mathcal{V}$ (that is, conjugate to $\mathcal{U}$ with respect to $B$ ) can be derived. The procedure to derive the conjugate entropy variables $\mathcal{V}$ to $\mathcal{U}=\{\boldsymbol{p}, \boldsymbol{F}, \boldsymbol{H}, J, E\}^{T}$ with respect to the ballistic energy was presented in [50] and is summarised in Appendix A for completeness.

Specifically, the ballistic free energy $B$ is defined in terms of the linear momentum $\boldsymbol{p}$, the triplet of deformation measures $\mathcal{X}$ and the total energy $E$ density as

$$
B(\boldsymbol{X}, t)=\hat{B}\left(\boldsymbol{p}, \boldsymbol{\mathcal { X }}_{E}\right)=E-\theta_{R} \hat{\eta}\left(\boldsymbol{p}, \boldsymbol{\mathcal { X }}_{E}\right)
$$

where $B(\boldsymbol{X}, t)$ and $\hat{B}\left(\boldsymbol{p}, \boldsymbol{\mathcal { X }}_{E}\right)$ represent alternative functional representations of the same magnitude. With this, the associated virtual work conjugates $\delta \mathcal{V}$ can then be obtained as

$$
\delta \mathcal{V}=\left[\begin{array}{c}
\delta \boldsymbol{\Gamma}_{\boldsymbol{v}} \\
\delta \boldsymbol{\Gamma}_{\boldsymbol{F}} \\
\delta \boldsymbol{\Gamma}_{\boldsymbol{H}} \\
\delta \boldsymbol{\Gamma}_{J} \\
\delta \boldsymbol{\Gamma}_{E}
\end{array}\right] ; \quad \mathcal{V}=\left[\begin{array}{c}
\boldsymbol{\Gamma}_{\boldsymbol{v}} \\
\boldsymbol{\Gamma}_{\boldsymbol{F}} \\
\boldsymbol{\Gamma}_{\boldsymbol{H}} \\
\boldsymbol{\Gamma}_{J} \\
\boldsymbol{\Gamma}_{E}
\end{array}\right]=\frac{\partial \hat{B}}{\partial \mathcal{U}}=\frac{\theta_{R}}{\theta}\left[\begin{array}{c}
\boldsymbol{v} \\
\boldsymbol{\Sigma}_{\boldsymbol{F}} \\
\boldsymbol{\Sigma}_{\boldsymbol{H}} \\
\boldsymbol{\Sigma}_{J} \\
\frac{\vartheta}{\theta_{R}}
\end{array}\right]
$$

where $\delta \boldsymbol{\Gamma}_{\boldsymbol{v}}$ is the virtual velocity field, $\left\{\delta \boldsymbol{\Gamma}_{\boldsymbol{F}}, \delta \boldsymbol{\Gamma}_{\boldsymbol{H}}, \delta \Gamma_{J}\right\}$ are appropriate virtual conjugate stresses to $\{\boldsymbol{F}, \boldsymbol{H}, J\}$ respectively, and $\delta \Gamma_{E}$ is the (dimensionless) energy conjugate, related to the temperature change $\vartheta=\theta-\theta_{R}$.

It is very interesting to realise that when considering an isothermal deformation process, that is $\theta=\theta_{R}$, it is easy to see that $\delta \mathcal{V}=\left[\delta \boldsymbol{v}, \delta \boldsymbol{\Sigma}_{\boldsymbol{F}}, \delta \boldsymbol{\Sigma}_{\boldsymbol{H}}, \delta \Sigma_{J}, 0\right]^{T}$, and hence, the energy equation (1e) decouples from the rest of the system when treated in a weak manner. This would then imply that the fields (34) degenerate to the usual work conjugates used for isothermal hyperelasticity [3] and the ballistic free energy reduces to the sum of the kinetic energy and the Helmholtz's free energy $\psi_{R}$, which was used as convex entropy function in previous work by the authors in the context of isothermal hyperelasticity 11 [1 3$]$.

Integrating by parts the last term on the right hand side of (33), and expanding the resulting equation yields

$$
\int_{V_{R}} \delta \mathcal{V} \bullet \frac{\partial \mathcal{U}}{\partial t} d V_{R}=\int_{V_{R}} \delta \mathcal{V} \bullet \mathcal{S} d V_{R}+\int_{V_{R}} \mathcal{F}_{I} \bullet \frac{\partial \delta \mathcal{V}}{\partial X_{I}} d V_{R}-\int_{\partial V_{R}} \delta \mathcal{V} \bullet \mathcal{F}_{\boldsymbol{N}} d A
$$

where the normal fluxes $\mathcal{F}_{N}=\mathcal{F}_{I} N_{I}$ with $N_{I}$ being the material outward normal in $I$-th material direction. Above representation (35) can be particularised to the case of the linear momentum $\boldsymbol{p}$, the triplet of geometric strain measures $\{\boldsymbol{F}, \boldsymbol{H}, J\}$ and the total energy density

\footnotetext{
${ }^{9}$ Not to be confused with the concept of entropy density $\eta$.

${ }^{10}$ The fields $\mathcal{V}$ are generally known as entropy conjugates because they are obtained from the calculation of the partial derivatives of the convex entropy function with respect to the conservation variables $\mathcal{U}$.

${ }^{11}$ This is easily seen because $\left.\hat{B}\left(\boldsymbol{p}, \mathcal{X}_{E}\right)\right|_{\theta=\theta_{R}}=\frac{1}{2 \rho_{R}} \boldsymbol{p} \cdot \boldsymbol{p}+\tilde{\mathcal{E}}_{R}(\mathcal{X})-\theta_{R} \tilde{\eta}_{R}(\mathcal{X})=\frac{1}{2 \rho_{R}} \boldsymbol{p} \cdot \boldsymbol{p}+\psi_{R}(\boldsymbol{\mathcal { X }})$.
} 
$E$ as

$$
\begin{aligned}
\int_{V_{R}} \delta \boldsymbol{\Gamma}_{\boldsymbol{v}} \cdot \frac{\partial \boldsymbol{p}}{\partial t} d V_{R} & =\int_{V_{R}}-\boldsymbol{P}: \boldsymbol{\nabla}_{0} \delta \boldsymbol{\Gamma}_{\boldsymbol{v}} d V_{R}+\int_{V_{R}} \delta \boldsymbol{\Gamma}_{\boldsymbol{v}} \cdot \boldsymbol{f}_{0} d V_{R}+\int_{\partial V_{R}} \delta \boldsymbol{\Gamma}_{\boldsymbol{v}} \cdot \boldsymbol{t}_{B} d A ; \\
\int_{V_{R}} \delta \boldsymbol{\Gamma}_{\boldsymbol{F}}: \frac{\partial \boldsymbol{F}}{\partial t} d V_{R} & =\int_{V_{R}} \delta \boldsymbol{\Gamma}_{\boldsymbol{F}}: \boldsymbol{\nabla}_{0} \boldsymbol{v} d V_{R} ; \\
\int_{V_{R}} \delta \boldsymbol{\Gamma}_{\boldsymbol{H}}: \frac{\partial \boldsymbol{H}}{\partial t} d V_{R} & =\int_{V_{R}} \delta \boldsymbol{\Gamma}_{\boldsymbol{H}}:\left(\boldsymbol{F} \times \boldsymbol{\nabla}_{0} \boldsymbol{v}\right) d V_{R} ; \\
\int_{V_{R}} \delta \Gamma_{J} \frac{\partial J}{\partial t} d V_{R} & =\int_{V_{R}} \delta \Gamma_{J} \boldsymbol{H}:\left(\boldsymbol{\nabla}_{0} \boldsymbol{v}\right) d V_{R} ; \\
\int_{V_{R}} \delta \Gamma_{E} \frac{\partial E}{\partial t} d V_{R} & =\int_{V_{R}}-\boldsymbol{v} \cdot \boldsymbol{P}\left(\boldsymbol{\nabla}_{0} \delta \Gamma_{E}\right) d V_{R}+\int_{V_{R}} \boldsymbol{Q} \cdot\left(\boldsymbol{\nabla}_{0} \delta \Gamma_{E}\right) d V_{R} \\
& +\int_{V_{R}} \delta \Gamma_{E}\left(\boldsymbol{v} \cdot \boldsymbol{f}_{R}+s_{R}\right) d V_{R}+\int_{\partial V_{R}} \delta \Gamma_{E}\left(\boldsymbol{v}_{B} \cdot \boldsymbol{t}_{B}-Q_{B}\right) d A .
\end{aligned}
$$

Notice that the main advantage of integrating by parts as shown in (35) is to enable the imposition of the physical boundary conditions. This is indeed useful for the linear momentum equation 36a and the total energy equation (36e) as both expressions introduce naturally the boundary tractions $\boldsymbol{t}_{B}$, the boundary velocity vector $\boldsymbol{v}_{B}$ and the heat flux $Q_{B}$, but less important in the case of geometric conservation laws. Hence, as shown in $(36 \mathrm{~b})-(36 \mathrm{~d})$, the update equations for the deformation measures $\{\boldsymbol{F}, \boldsymbol{H}, J\}$ are obtained without resorting to integration by parts, and using the non-conservative form of the evolution equations (3a) and (3b).

Remark 2: Following Remark 1, an alternative weak form statement to that of 36e can be formulated by regarding the entropy density $\eta$ as the thermal unknown (instead of the total energy density $E$ ). In this case, the weak form of the entropy equation 15 is presented as

$$
\int_{V_{R}} \delta \vartheta \frac{\partial \eta}{\partial t} d V_{R}=-\int_{\partial V_{R}} \delta \vartheta\left(\frac{Q_{B}}{\theta_{B}}\right) d A+\int_{V_{R}}\left(\frac{\boldsymbol{Q}}{\theta}\right) \cdot \nabla_{0} \delta \vartheta d V_{R}+\int_{V_{R}} \delta \vartheta\left(\frac{r_{R}}{\theta}-\frac{1}{\theta^{2}} \boldsymbol{Q} \cdot \nabla_{0} \theta\right) d V_{R}
$$

where $\delta \vartheta=\delta \theta$ is the virtual conjugate field of the entropy $\eta$ with respect to the ballistic energy $B$ (that is, $\vartheta=\partial B / \partial \eta$ ). Above weak statement (37) can be used instead of (36e) when solving the system of conservation laws.

\subsection{Second law of thermodynamics}

It is instructive to revisit the second law of thermodynamics when written in terms of the ballistic free energy. The time rate of the volume integral of the ballistic free energy is obtained 
as follows

$$
\begin{aligned}
\frac{d}{d t} \int_{V_{R}} B d V_{R} & =\int_{V_{R}} \mathcal{V} \bullet \frac{\partial \boldsymbol{U}}{\partial t} d V_{R} \\
& =\int_{V_{R}}\left(\boldsymbol{\Gamma}_{\boldsymbol{v}} \cdot \frac{\partial \boldsymbol{p}}{\partial t}+\boldsymbol{\Gamma}_{\boldsymbol{F}}: \frac{\partial \boldsymbol{F}}{\partial t}+\boldsymbol{\Gamma}_{\boldsymbol{H}}: \frac{\partial \boldsymbol{H}}{\partial t}+\Gamma_{J} \frac{\partial J}{\partial t}+\Gamma_{E} \frac{\partial E}{\partial t}\right) d V_{R} \\
& =\int_{V_{R}}\left(\frac{\theta_{R}}{\theta}\left(\boldsymbol{v} \cdot \frac{\partial \boldsymbol{p}}{\partial t}+\boldsymbol{\Sigma}_{\boldsymbol{F}}: \frac{\partial \boldsymbol{F}}{\partial t}+\boldsymbol{\Sigma}_{\boldsymbol{H}}: \frac{\partial \boldsymbol{H}}{\partial t}+\Sigma_{J} \frac{\partial J}{\partial t}\right)+\frac{\vartheta}{\theta} \frac{\partial E}{\partial t}\right) d V_{R} \\
& =\int_{V_{R}}\left(\frac{\theta_{R}}{\theta}\left(\boldsymbol{v} \cdot \frac{\partial \boldsymbol{p}}{\partial t}+\left(\boldsymbol{\Sigma}_{\boldsymbol{F}}+\boldsymbol{\Sigma}_{\boldsymbol{H}} \times \boldsymbol{F}+\Sigma_{J} \boldsymbol{H}\right): \boldsymbol{\nabla}_{0} \boldsymbol{v}\right)+\frac{\vartheta}{\theta} \frac{\partial E}{\partial t}\right) d V_{R} \\
& =\int_{V_{R}}\left(\frac{\theta_{R}}{\theta}\left(\boldsymbol{v} \cdot \frac{\partial \boldsymbol{p}}{\partial t}+\boldsymbol{P}: \boldsymbol{\nabla}_{0} \boldsymbol{v}\right)+\frac{\vartheta}{\theta} \frac{\partial E}{\partial t}\right) d V_{R}
\end{aligned}
$$

where, in the first line of $(38)$, use has been made of the conjugacy of the fields $\mathcal{U}$ and $\mathcal{V}$ (refer to Appendix A). In addition, equations (3a) and (3b) have been substituted in the fourth line of (38). Finally, equation (12) has been substituted in the last line of (38). Subsequently, we can substitute the linear momentum and total energy conservation laws (1a) and (1e), into (38) to give

$$
\frac{d}{d t} \int_{V_{R}} B d V_{R}=\int_{V_{R}}\left(\frac{\theta_{R}}{\theta}\left(\boldsymbol{f}_{R} \cdot \boldsymbol{v}+\operatorname{DIV}\left(\boldsymbol{P}^{T} \boldsymbol{v}\right)\right)+\frac{\vartheta}{\theta}\left(\boldsymbol{f}_{R} \cdot \boldsymbol{v}+s_{R}+\mathrm{DIV}\left(\boldsymbol{P}^{T} \boldsymbol{v}-\boldsymbol{Q}\right)\right)\right) d V_{R},
$$

which can be re-written as

$$
\frac{d}{d t} \int_{V_{R}} B d V_{R}=\int_{V_{R}}\left(\boldsymbol{f}_{R} \cdot \boldsymbol{v}+\operatorname{DIV}\left(\boldsymbol{P}^{T} \boldsymbol{v}\right)\right) d V_{R}+\int_{V_{R}} \frac{\vartheta}{\theta}\left(s_{R}-\operatorname{DIV}(\boldsymbol{Q})\right) d V_{R}
$$

By performing integration by parts of the DIV terms in equation 40 and after some rearrangement, it yields

$$
\frac{d}{d t} \int_{V_{R}} B d V_{R}-\dot{\Pi}_{\mathrm{ext}}-\mathcal{Q}_{\mathrm{ext}}=\int_{V_{R}} \frac{\theta_{R}}{\theta^{2}} \boldsymbol{Q} \cdot \nabla_{0} \theta d V_{R}
$$

where $\dot{\Pi}_{\text {ext }}$ denotes the power introduced by mechanical forces, defined as

$$
\dot{\Pi}_{\mathrm{ext}}=\int_{V_{R}} \boldsymbol{f}_{R} \cdot \boldsymbol{v} d V_{R}+\int_{\partial V_{R}} \boldsymbol{v} \cdot \boldsymbol{t}_{B} d A,
$$

and $\mathcal{Q}_{\text {ext }}$ represents the heat source and heat flux added (removed) to (from) the system, defined as

$$
\mathcal{Q}_{\text {ext }}=\int_{V_{R}} \frac{\vartheta}{\theta} s_{R} d V_{R}-\int_{\partial V_{R}} \frac{\vartheta}{\theta} Q_{B} d A .
$$

Noticing in above equation (41) that the right hand side is always non-positive (i.e. $\boldsymbol{Q} \cdot \boldsymbol{\nabla}_{0} \theta \leq$ 0 ), equation (41) can be transformed into the following inequality

$$
\frac{d}{d t} \int_{V_{R}} B d V_{R}-\dot{\Pi}_{\mathrm{ext}}-\mathcal{Q}_{\mathrm{ext}} \leq 0,
$$

which represents a valid expression for the second law of thermodynamics. Satisfaction of inequality (44) is a necessary ab initio condition to ensure stability, otherwise referred to as 
the Coleman-Noll procedure [66]. In the special case of an isolated system (i.e. $\dot{\Pi}_{\text {ext }}=0$ and $\mathcal{Q}_{\text {ext }}=0$ ), inequality (44) reduces to

$$
\frac{d}{d t} \int_{V_{R}} B d V_{R} \leq 0
$$

This means that for an isolated system, the decrease in ballistic free energy is intrinsically linked to the dissipation introduced by any inelastic effects. This key concept will be further exploited in the following section at a semi-discrete level. Finally, it is interesting to see that, in the case of an isothermal process $\theta=\theta_{R}$ (i.e. the ballistic free energy becomes the sum of the kinetic energy $\frac{1}{2 \rho_{R}} \boldsymbol{p} \cdot \boldsymbol{p}$ and the Helmholtz's free energy $\psi_{R}$ ), above inequality reduces to 67

$$
\frac{d}{d t} \int_{V_{R}}\left(\frac{1}{2 \rho_{R}} \boldsymbol{p} \cdot \boldsymbol{p}+\psi_{R}\right) d V_{R}-\dot{\Pi}_{\mathrm{ext}} \leq 0
$$

Remark 3: Alternatively, if the entropy $\eta$ is used as the thermal unknown (instead of the total energy $E$ ), the time rate of the ballistic free energy $B$ can also be computed as

$$
\begin{aligned}
\frac{d}{d t} \int_{V_{R}} B d V_{R} & =\int_{V_{R}} \mathcal{V} \bullet \frac{\partial \mathcal{U}}{\partial t} d V_{R} \\
& =\int_{V_{R}}\left(\boldsymbol{v} \cdot \frac{\partial \boldsymbol{p}}{\partial t}+\boldsymbol{\Sigma}_{\boldsymbol{F}}: \frac{\partial \boldsymbol{F}}{\partial t}+\boldsymbol{\Sigma}_{\boldsymbol{H}}: \frac{\partial \boldsymbol{H}}{\partial t}+\Sigma_{J} \frac{\partial J}{\partial t}+\vartheta \frac{\partial \eta}{\partial t}\right) d V_{R}
\end{aligned}
$$

where it can be noticed the alternative set of entropy conjugate fields $\left\{\boldsymbol{v}, \boldsymbol{\Sigma}_{\boldsymbol{F}}, \boldsymbol{\Sigma}_{\boldsymbol{H}}, \Sigma_{J}, \vartheta\right\}$, obtained as the partial derivative of the ballistic free energy $B$ with respect to the conservation variables $\{\boldsymbol{p}, \boldsymbol{F}, \boldsymbol{H}, J, \eta\}$. Use of the geometric conservation laws (1b) to (1d) and equation (12) into (47) results in

$$
\frac{d}{d t} \int_{V_{R}} B d V_{R}=\int_{V_{R}}\left(\left(\boldsymbol{v} \cdot \frac{\partial \boldsymbol{p}}{\partial t}+\boldsymbol{P}: \nabla_{0} \boldsymbol{v}\right)+\vartheta \frac{\partial \eta}{\partial t}\right) d V_{R}
$$

Substitution of the linear momentum (1a) and entropy conservation law (15) into (48), yields

$$
\frac{d}{d t} \int_{V_{R}} B d V_{R}=\int_{V_{R}}\left(\left(\boldsymbol{f}_{R} \cdot \boldsymbol{v}+\operatorname{DIV}\left(\boldsymbol{P}^{T} \boldsymbol{v}\right)\right)+\vartheta\left(\frac{s_{R}}{\theta}-\operatorname{DIV}\left(\frac{\boldsymbol{Q}}{\theta}\right)-\frac{1}{\theta^{2}} \boldsymbol{Q} \cdot \boldsymbol{\nabla}_{0} \theta\right)\right) d V_{R}
$$

which, after some algebraic manipulation of the last term within the integrand of the right hand side, equation (49) reduces to above equation (40) and thus, inequality (44).

\section{Spatial discretisation}

Consider the thermo-elastic body is described (discretised) by a cloud of particles as shown in Figure 1. For the case of corrected SPH methods [1 푸, 6, 17], both the problem variables $\mathcal{U}$ and the conjugate pairs $\delta \mathcal{V}$ are in general interpolated at any given position via corrected kernel (or smoothing) functions $\tilde{W}$ with a given compact support around every particle. Specifically, for a given position $\boldsymbol{X}_{a}$, both $\mathcal{U}$ and $\delta \mathcal{V}$ can be approximated as

$$
\mathcal{U}_{a}(t) \approx \sum_{b \in \Lambda_{a}^{b}} \mathrm{~N}_{b}\left(\boldsymbol{X}_{a}\right) \mathcal{U}_{b}(t) ; \quad \delta \mathcal{V}_{a} \approx \sum_{b \in \Lambda_{a}^{b}} \mathrm{~N}_{b}\left(\boldsymbol{X}_{a}\right) \delta \mathcal{V}_{b} ; \quad \mathrm{N}_{b}\left(\boldsymbol{X}_{a}\right)=V_{R}^{b} \tilde{W}_{b}\left(\boldsymbol{X}_{a}\right)
$$




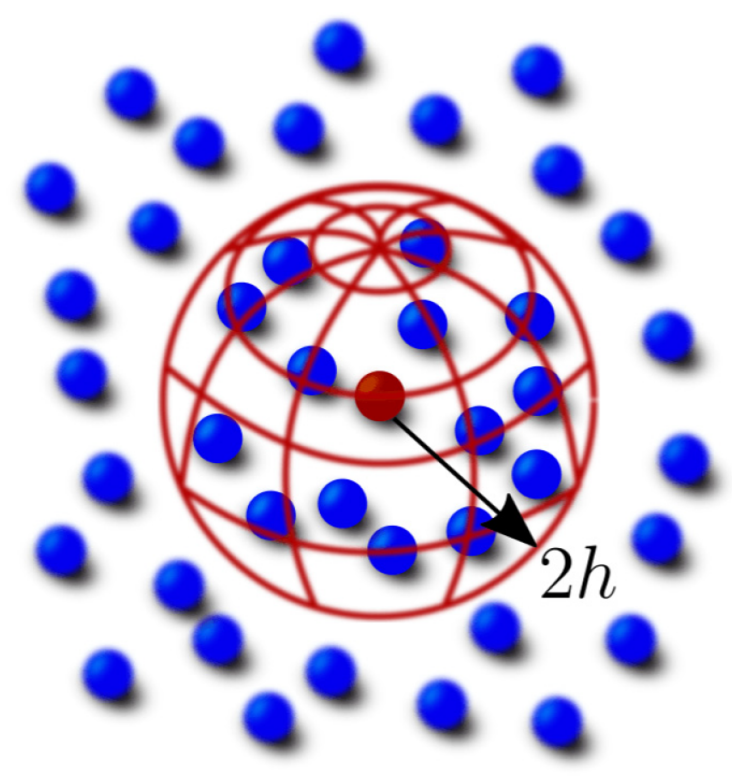

Figure 1: Particle approximation

Here, $\Lambda_{a}^{b}$ represents the set of neighbouring particles $b$ that lie inside a sphere of a given radius around $\boldsymbol{X}_{a}, V_{R}^{b}$ represents the volume associated to particle $b$ and $\mathcal{U}_{b}(t)$ and $\delta \mathcal{V}_{b}$ represent the time varying variables and their virtual conjugate pairs stored at particle $b$, respectively. In addition, $[\bullet]_{a}(t)$ represent the problem variables at position $\boldsymbol{X}_{a}$ and time $t$, that is $[\bullet]_{a}(t)=[\bullet]\left(\boldsymbol{X}_{a}, t\right)$. Additionally, the use of (corrected) SPH shape functions $\mathbf{N}$ ensure that both constant and linear functions are perfectly interpolated [7].

In addition, for the evaluation of the material gradient $\nabla_{0}(\bullet)$ of any arbitrary vector function $\boldsymbol{f}$, we employ the well-known gradient correction [1] described as follows

$$
\boldsymbol{\nabla}_{0} \boldsymbol{f}\left(\boldsymbol{X}_{a}\right) \approx \sum_{b \in \Lambda_{a}^{b}}\left(\boldsymbol{f}_{b}-\boldsymbol{f}_{a}\right) \otimes \boldsymbol{G}_{b}\left(\boldsymbol{X}_{a}\right) ; \quad \boldsymbol{G}_{b}\left(\boldsymbol{X}_{a}\right)=V_{R}^{b} \tilde{\boldsymbol{\nabla}}_{0} W_{b}\left(\boldsymbol{X}_{a}\right) ; \quad \tilde{\boldsymbol{\nabla}}_{0} W_{b}\left(\boldsymbol{X}_{a}\right)=\boldsymbol{L}_{a} \boldsymbol{\nabla}_{0} W_{b}\left(\boldsymbol{X}_{a}\right),
$$

where $\boldsymbol{L}_{a}$ is explicitly evaluated as [7]

$$
\boldsymbol{L}_{a}=\left(\sum_{b \in \Lambda_{a}^{b}} V_{R}^{b} \nabla_{0} W_{b}\left(\boldsymbol{X}_{a}\right) \otimes\left(\boldsymbol{X}_{b}-\boldsymbol{X}_{a}\right)\right)^{-1} .
$$

In this expression, the use of the kernel gradient correction $\tilde{\nabla}_{0}$ ensures the gradient of any linear field distribution is exactly evaluated. This type of kernel gradient correction has been extensively discussed in References [7, 10]. Interestingly, by defining the vector $\mathbf{C}_{a b}:=$ $2 V_{R}^{a} \boldsymbol{G}_{b}\left(\boldsymbol{X}_{a}\right)$ [3], we can now re-write above expression (51) to give

$$
\boldsymbol{\nabla}_{0} \boldsymbol{f}\left(\boldsymbol{X}_{a}\right) \approx \frac{1}{V_{R}^{a}} \sum_{b \in \Lambda_{a}^{b}} \frac{1}{2}\left(\boldsymbol{f}_{b}-\boldsymbol{f}_{a}\right) \otimes \mathbf{C}_{a b} .
$$

Its reciprocal relationship can also be defined as $\mathbf{C}_{b a}=2 V_{R}^{b} \boldsymbol{G}_{a}\left(\boldsymbol{X}_{b}\right)$ where $\boldsymbol{G}_{a}\left(\boldsymbol{X}_{b}\right)=$ $V_{R}^{a} \tilde{\boldsymbol{\nabla}}_{0} W_{a}\left(\boldsymbol{X}_{b}\right)$. Due to the anti-symmetric nature of the SPH gradient correction [3], notice here that $\boldsymbol{G}_{b}\left(\boldsymbol{X}_{a}\right) \neq-\boldsymbol{G}_{a}\left(\boldsymbol{X}_{b}\right)$ which in turn lead to $\mathbf{C}_{a b} \neq-\mathbf{C}_{b a}$. 


\subsection{SPH semi-discrete equations}

Substituting the expressions for the corrected SPH shape functions $(50)$ (for $\mathcal{U}$ and $\delta \mathcal{V}$ ) and using the corrected gradient evaluations (53) (for $\boldsymbol{\nabla}_{0} \delta \mathcal{V}$ ) into (35), the SPH discretisation for the system $\{\boldsymbol{p}, \boldsymbol{F}, \boldsymbol{H}, J, E\}$ described in 36 becomes, after some algebraic manipulations, ${ }^{12}$

$$
\begin{aligned}
V_{R}^{a} \frac{d \boldsymbol{p}_{a}}{d t} & =\boldsymbol{T}_{a}+V_{R}^{a} \boldsymbol{f}_{R}^{a}+A_{R}^{a} \boldsymbol{t}_{B}^{a} \\
V_{R}^{a} \frac{d \boldsymbol{F}_{a}}{d t} & =\sum_{b \in \Lambda_{a}^{b}}\left(\frac{\boldsymbol{v}_{b}-\boldsymbol{v}_{a}}{2}\right) \otimes \mathbf{C}_{a b} \\
V_{R}^{a} \frac{d \boldsymbol{H}_{a}}{d t} & =\boldsymbol{F}_{a} \times\left(\sum_{b \in \Lambda_{a}^{b}}\left(\frac{\boldsymbol{v}_{b}-\boldsymbol{v}_{a}}{2}\right) \otimes \mathbf{C}_{a b}\right) \\
V_{R}^{a} \frac{d J_{a}}{d t} & =\boldsymbol{H}_{a}:\left(\sum_{b \in \Lambda_{a}^{b}}\left(\frac{\boldsymbol{v}_{b}-\boldsymbol{v}_{a}}{2}\right) \otimes \mathbf{C}_{a b}\right)+\sum_{b \in \Lambda_{a}^{b}} \mathcal{D}_{J}^{a b} ; \\
V_{R}^{a} \frac{d E_{a}}{d t} & =\sum_{b \in \Lambda_{a}^{b}} \frac{1}{2}\left[\left(\boldsymbol{P}_{a} \mathbf{C}_{a b}\right) \cdot \boldsymbol{v}_{b}-\left(\boldsymbol{P}_{b} \mathbf{C}_{b a}\right) \cdot \boldsymbol{v}_{a}\right]-\sum_{b \in \Lambda_{a}^{b}} \frac{1}{2}\left[\left(\frac{\boldsymbol{Q}_{a}}{\theta_{a}} \cdot \mathbf{C}_{a b}\right) \theta_{b}-\left(\frac{\boldsymbol{Q}_{b}}{\theta_{b}} \cdot \mathbf{C}_{b a}\right) \theta_{a}\right] \\
& +V_{R}^{a}\left(\boldsymbol{f}_{R}^{a} \cdot \boldsymbol{v}_{a}+s_{R}^{a}\right)+A_{R}^{a}\left(\boldsymbol{t}_{B}^{a} \cdot \boldsymbol{v}_{B}^{a}-Q_{B}^{a}\right)+\sum_{b \in \Lambda_{a}^{b}} \mathcal{D}_{E}^{a b} .
\end{aligned}
$$

Here, the representation for the (algorithmic) internal nodal force $\boldsymbol{T}_{a}$ is defined as

$$
\boldsymbol{T}_{a}=\sum_{b \in \Lambda_{a}^{b}} \frac{1}{2}\left(\boldsymbol{P}_{a} \mathbf{C}_{a b}-\boldsymbol{P}_{b} \mathbf{C}_{b a}\right)+\sum_{b \in \Lambda_{a}^{b}} \mathcal{D}_{\boldsymbol{p}}^{a b}
$$

The heat flux evaluation at particle $a$ is approximated via $(53)$ as

$$
\boldsymbol{Q}_{a}=-\boldsymbol{K}_{a} \boldsymbol{\nabla}_{0} \theta\left(\boldsymbol{X}_{a}, t\right) \approx-\frac{1}{V_{R}^{a}} \sum_{b \in \Lambda_{a}^{b}} \frac{1}{2}\left(\theta_{b}-\theta_{a}\right) \boldsymbol{K}_{a} \mathbf{C}_{a b} ; \quad \boldsymbol{K}_{a}=J_{a}^{-1} h_{a} \boldsymbol{H}_{a}^{T} \boldsymbol{H}_{a}
$$

where the same heat flux approximation (56) also holds for particle $b$ (just by renaming particle $a$ as particle $b$ ).

In expression (54a), $A_{R}^{a}$ represent the material tributary area and $\boldsymbol{t}_{B}^{a}$ its traction vector computed directly from the given traction boundary conditions. Notice that in (54a), $A_{R}^{a}=0$ for those particles not placed on the boundary.

Finally, the remaining terms to be defined in equations (54a), (54d) and (54e) are the socalled numerical dissipation terms $\left\{\mathcal{D}_{p}^{a b}, \mathcal{D}_{J}^{a b}, \mathcal{D}_{E}^{a b}\right\}$. These terms can be derived using the semidiscrete version of the classical Coleman-Noll procedure [66] in order to ensure the production of numerical entropy. This will be presented in the following section. For the isothermal case, equation (54e) is not strictly needed and equations $(54 \mathrm{a})-(54 \mathrm{~d})$ reduce to the semi-discrete $\mathrm{SPH}$ scheme already explored by the authors in [1-3].

Notice here that the stabilisation term applied to the linear momentum evolution (54a) alleviates the appearance of spurious zero-energy (hourglass-like [1]) modes due to rank deficiency

\footnotetext{
${ }^{12}$ For an in-depth derivation, the reader is referred to Sections 3.3.1 and 3.3.2 in previous contribution [1] by the authors.
} 
inherent to the use of (nodal) particle integration, whereas the stabilisation in the volume map evolution (54d) addresses pressure instabilities (especially, in near incompressibility). Additionally, it is also interesting to show the possible addition of stabilisation to the total energy equation (54e).

Remark 4: Following Remark 2, an alternative SPH scheme can be formulated where the semi-discrete form of equation (37) gives, after some algebra

$$
V_{R}^{a} \frac{d \eta_{a}}{d t}=-\sum_{b \in \Lambda_{a}^{b}} \frac{1}{2}\left(\frac{\boldsymbol{Q}_{a}}{\theta_{a}} \mathbf{C}_{a b}-\frac{\boldsymbol{Q}_{b}}{\theta_{b}} \mathbf{C}_{b a}\right)-\frac{\boldsymbol{Q}_{a}}{\theta_{a}^{2}} \cdot \sum_{b \in \Lambda_{a}^{b}} \frac{1}{2}\left(\theta_{b}-\theta_{a}\right) \mathbf{C}_{a b}+V_{R}^{a} \frac{s_{R}^{a}}{\theta_{a}}-\frac{Q_{B}^{a}}{\theta_{B}^{a}} A_{R}^{a}+\sum_{b \in \Lambda_{a}^{b}} \mathcal{D}_{\eta}^{a b},
$$

where $\eta_{a}$ represents the entropy stored at particle $a$ and $\mathrm{D}_{\eta}^{a b}$ denotes the associated numerical dissipation.

\subsection{Numerical entropy production}

In this section, inequality (44) is assessed for the above set of SPH semi-discrete equations (54). Specifically, the semi-discrete counterpart of (38) is

$$
\begin{aligned}
\sum_{a} V_{R}^{a} \frac{d B_{a}}{d t} & =\sum_{a} V_{R}^{a} \mathcal{V}_{a} \cdot \frac{d \mathcal{U}_{a}}{d t} \\
& =\sum_{a} V_{R}^{a}\left[\frac{\theta_{R}}{\theta_{a}}\left(\boldsymbol{v}_{a} \cdot \frac{d \boldsymbol{p}_{a}}{d t}+\boldsymbol{\Sigma}_{\boldsymbol{F}}^{a}: \frac{d \boldsymbol{F}_{a}}{d t}+\Sigma_{\boldsymbol{H}}^{a}: \frac{d \boldsymbol{H}_{a}}{d t}+\Sigma_{J}^{a} \frac{d J_{a}}{d t}\right)+\frac{\vartheta_{a}}{\theta_{a}} \frac{d E_{a}}{d t}\right] \\
& =\sum_{a} V_{R}^{a}\left[\frac{\theta_{R}}{\theta_{a}}\left(\boldsymbol{v}_{a} \cdot \frac{d \boldsymbol{p}_{a}}{d t}+\boldsymbol{P}_{a}: \frac{d \boldsymbol{F}_{a}}{d t}\right)+\frac{\vartheta_{a}}{\theta_{a}} \frac{d E_{a}}{d t}\right]
\end{aligned}
$$

where $\vartheta_{a}=\theta_{a}-\theta_{R}$ represents the change in temperature at particle $a$ with respect to the reference temperature. Subsequently, we can substitute the linear momentum (54a), the deformation gradient (54b) and total energy conservation laws (54e) into (58) and, after some algebra, gives

$$
\begin{aligned}
\sum_{a} V_{R}^{a} \frac{d B_{a}}{d t} & =\sum_{a} \sum_{b \in \Lambda_{a}^{b}} \frac{1}{2}\left[\left(\boldsymbol{P}_{a} \mathbf{C}_{a b}\right) \cdot \boldsymbol{v}_{b}-\left(\boldsymbol{P}_{b} \mathbf{C}_{b a}\right) \cdot \boldsymbol{v}_{a}\right]-\sum_{a} \sum_{b \in \Lambda_{a}^{b}} \frac{1}{2}\left[\left(\frac{\boldsymbol{Q}_{a}}{\theta_{a}} \cdot \mathbf{C}_{a b}\right) \theta_{b}-\left(\frac{\boldsymbol{Q}_{b}}{\theta_{b}} \cdot \mathbf{C}_{b a}\right) \theta_{a}\right] \\
& +\sum_{a} \sum_{b \in \Lambda_{a}^{b}} \frac{\theta_{R}}{2 \theta_{a}}\left[\left(\frac{\boldsymbol{Q}_{a}}{\theta_{a}} \cdot \mathbf{C}_{a b}\right) \theta_{b}-\left(\frac{\boldsymbol{Q}_{b}}{\theta_{b}} \cdot \mathbf{C}_{b a}\right) \theta_{a}\right]+\sum_{a} \sum_{b \in \Lambda_{a}^{b}} \mathcal{D}_{E}^{a b} \\
& -\sum_{a} \sum_{b \in \Lambda_{a}^{b}} \frac{\theta_{R}}{\theta_{a}}\left[\mathcal{D}_{E}^{a b}-\boldsymbol{v}_{a} \cdot \mathcal{D}_{\boldsymbol{p}}^{a b}-\Sigma_{J}^{a} \mathcal{D}_{J}^{a b}\right]+\dot{\Pi}_{\mathrm{ext}}+\mathcal{Q}_{\mathrm{ext}} .
\end{aligned}
$$

Here, $\dot{\Pi}_{\text {ext }}$ and $\mathcal{Q}_{\text {ext }}$ denote the semi-discrete power and total heat contribution, respectively, expressed as

$$
\dot{\Pi}_{\mathrm{ext}}=\sum_{a}\left(V_{R}^{a} \boldsymbol{v}_{a} \cdot \boldsymbol{f}_{R}^{a}+A_{R}^{a} \boldsymbol{t}_{B}^{a} \cdot \boldsymbol{v}_{B}^{a}\right) ; \quad \mathcal{Q}_{\mathrm{ext}}=\sum_{a} \frac{\vartheta_{a}}{\theta_{a}}\left(V_{R}^{a} s_{R}^{a}-A_{R}^{a} Q_{B}^{a}\right) .
$$

In the current paper, we strongly enforce local conservation for the pair-wise stabilisation terms, in such a way that $\mathcal{D}_{E}^{a b}=-\mathcal{D}_{E}^{b a}, \mathcal{D}_{\boldsymbol{p}}^{a b}=-\mathcal{D}_{\boldsymbol{p}}^{b a}$ and $\mathcal{D}_{J}^{a b}=-\mathcal{D}_{J}^{b a}$. By doing this the second 
term in the second line of (59) vanishes as a result of global conservation. Additionally, by noticing the nested summation carried out over particles in (59) and the anti-symmetric nature of the two terms in the first line of the right hand side, we can conclude that these terms cancel and thus (59) reduces to

$$
\begin{aligned}
\sum_{a} V_{R}^{a} \frac{d B_{a}}{d t}-\dot{\Pi}_{\mathrm{ext}}-\mathcal{Q}_{\mathrm{ext}} & =\sum_{a} \sum_{b \in \Lambda_{a}^{b}} \frac{\theta_{R}}{2 \theta_{a}}\left[\left(\frac{\boldsymbol{Q}_{a}}{\theta_{a}} \cdot \mathbf{C}_{a b}\right) \theta_{b}-\left(\frac{\boldsymbol{Q}_{b}}{\theta_{b}} \cdot \mathbf{C}_{b a}\right) \theta_{a}\right] \\
& -\underbrace{\sum_{a} \sum_{b \in \Lambda_{a}^{b}} \frac{\theta_{R}}{\theta_{a}}\left[\mathcal{D}_{E}^{a b}-\boldsymbol{v}_{a} \cdot \mathcal{D}_{\boldsymbol{p}}^{a b}-\Sigma_{J}^{a} \mathcal{D}_{J}^{a b}\right]}_{\mathcal{D}_{\text {total }}^{E}} .
\end{aligned}
$$

It is now the objective to demonstrate that both terms on the right hand side of (61) are non-positive (to be in agreement with inequality (44)). With respect to the first,

$$
\begin{aligned}
\sum_{a} \sum_{b \in \Lambda_{a}^{b}} \frac{\theta_{R}}{2 \theta_{a}}\left[\left(\frac{\boldsymbol{Q}_{a}}{\theta_{a}} \cdot \mathbf{C}_{a b}\right) \theta_{b}-\left(\frac{\boldsymbol{Q}_{b}}{\theta_{b}} \cdot \mathbf{C}_{b a}\right) \theta_{a}\right] & =\sum_{a} \sum_{b \in \Lambda_{a}^{b}} \frac{\theta_{R}}{2 \theta_{a}}\left[\left(\frac{\boldsymbol{Q}_{a}}{\theta_{a}} \cdot \mathbf{C}_{a b}\right) \theta_{b}-\left(\frac{\boldsymbol{Q}_{a}}{\theta_{a}} \cdot \mathbf{C}_{a b}\right) \theta_{a}\right] \\
& =\sum_{a} V_{R}^{a} \frac{\theta_{R}}{\theta_{a}^{2}} \boldsymbol{Q}_{a} \cdot\left[\frac{1}{V_{R}^{a}} \sum_{b \in \Lambda_{a}^{b}} \frac{1}{2}\left(\theta_{b}-\theta_{a}\right) \mathbf{C}_{a b}\right] \\
& =\sum_{a} V_{R}^{a} \frac{\theta_{R}}{\theta_{a}^{2}} \boldsymbol{Q}_{a} \cdot \nabla_{0} \theta\left(\boldsymbol{X}_{a}, t\right) \leq 0
\end{aligned}
$$

where the last inequality is fulfilled due to the definition of the conductive heat flux.

As for the last term on the right hand side of (61) (namely $\mathcal{D}_{\text {total }}^{E}$ ), and with the use of $\Xi_{a, b}=\theta_{a, b} / \theta_{R}$, this term can be equivalently written by swapping indices $a$ and $b$ to give

$$
\begin{aligned}
\mathcal{D}_{\text {total }}^{E} & =\sum_{a} \sum_{b \in \Lambda_{a}^{b}} \frac{1}{\Xi_{a}}\left[\mathcal{D}_{E}^{a b}-\boldsymbol{v}_{a} \cdot \mathcal{D}_{\boldsymbol{p}}^{a b}-\Sigma_{J}^{a} \mathcal{D}_{J}^{a b}\right] \\
& =\sum_{a} \sum_{b \in \Lambda_{a}^{b}} \frac{1}{\Xi_{b}}\left[\mathcal{D}_{E}^{b a}-\boldsymbol{v}_{b} \cdot \mathcal{D}_{p}^{b a}-\Sigma_{J}^{b} \mathcal{D}_{J}^{b a}\right] .
\end{aligned}
$$

Adding the first line and the second line of the equation above, and noting the antisymmetric nature of the stabilisation terms (e.g. $\mathcal{D}_{E}^{b a}=-\mathcal{D}_{E}^{a b}, \mathcal{D}_{\boldsymbol{p}}^{b a}=-\mathcal{D}_{\boldsymbol{p}}^{a b}$ and $\left.\mathcal{D}_{J}^{b a}=-\mathcal{D}_{J}^{a b}\right)$, an alternative expression for $\mathcal{D}_{\text {total }}^{E}$ is

$$
\mathcal{D}_{\text {total }}^{E}=\frac{1}{2} \sum_{a} \sum_{b \in \Lambda_{a}^{b}} \mathcal{D}_{\text {total }, a b}^{E}
$$

with

$$
\mathcal{D}_{\text {total }, a b}^{E}=\left(\frac{\boldsymbol{v}_{b}}{\Xi_{b}}-\frac{\boldsymbol{v}_{a}}{\Xi_{a}}\right) \cdot \mathcal{D}_{\boldsymbol{p}}^{a b}+\left(\frac{\Sigma_{J}^{b}}{\Xi_{b}}-\frac{\Sigma_{J}^{a}}{\Xi_{a}}\right) \mathcal{D}_{J}^{a b}+\left(\frac{1}{\Xi_{a}}-\frac{1}{\Xi_{b}}\right) \mathcal{D}_{E}^{a b} .
$$

Dissipation terms $\left\{\mathcal{D}_{\boldsymbol{p}}^{a b}, \mathcal{D}_{J}^{a b}, \mathcal{D}_{E}^{a b}\right\}$ remain to be defined in order to ensure non-negative entropy production. Sufficient conditions to guarantee this (and hence, the fulfilment of the second law 
of thermodynamics, namely $\mathcal{D}_{\text {total }}^{E} \geq 0$ (63), are given by

$$
\begin{aligned}
& \mathcal{D}_{\boldsymbol{p}}^{a b}=\boldsymbol{S}_{a b}^{\boldsymbol{p}}\left(\frac{\boldsymbol{v}_{b}}{\Xi_{b}}-\frac{\boldsymbol{v}_{a}}{\Xi_{a}}\right) ; \\
& \mathcal{D}_{J}^{a b}=S_{a b}^{J}\left(\frac{\Sigma_{J}^{b}}{\Xi_{b}}-\frac{\Sigma_{J}^{a}}{\Xi_{a}}\right) ; \\
& \mathcal{D}_{E}^{a b}=S_{a b}^{E}\left(\frac{1}{\Xi_{a}}-\frac{1}{\Xi_{b}}\right),
\end{aligned}
$$

with $\boldsymbol{S}_{a b}^{p}$ being defined as a positive semi-definite stabilisation matrix and $\left\{S_{a b}^{J}, S_{a b}^{E}\right\} \geq 0$. It is very interesting to observe how the dissipation terms $\left\{\mathcal{D}_{\boldsymbol{p}}^{a b}, \mathcal{D}_{J}^{a b}, \mathcal{D}_{E}^{a b}\right\}$ are directly related to the difference in velocity, stresses and temperature between interacting particles, typical of Riemann solver based upwinding terms [59].

Remark 5: Alternatively, inequality (44) also holds for the entropy based counterpart $\{\boldsymbol{p}, \boldsymbol{F}, \boldsymbol{H}, J, \eta\}$. Following the same procedure carried out above, the semi-discrete version of ballistic free energy in this case becomes

$$
\begin{aligned}
\sum_{a} V_{R}^{a} \frac{d B_{a}}{d t} & =\sum_{a} V_{R}^{a} \mathcal{V}_{a} \cdot \frac{d \mathcal{U}_{a}}{d t} \\
& =\sum_{a} V_{R}^{a}\left(\boldsymbol{v}_{a} \cdot \frac{d \boldsymbol{p}_{a}}{d t}+\boldsymbol{\Sigma}_{\boldsymbol{F}}^{a}: \frac{d \boldsymbol{F}_{a}}{d t}+\boldsymbol{\Sigma}_{\boldsymbol{H}}^{a}: \frac{d \boldsymbol{H}_{a}}{d t}+\Sigma_{J}^{a} \frac{d J_{a}}{d t}+\vartheta_{a} \frac{d \eta_{a}}{d t}\right) \\
& =\sum_{a} V_{R}^{a}\left(\boldsymbol{v}_{a} \cdot \frac{d \boldsymbol{p}_{a}}{d t}+\boldsymbol{P}_{a}: \frac{d \boldsymbol{F}_{a}}{d t}+\vartheta_{a} \frac{d \eta_{a}}{d t}\right),
\end{aligned}
$$

where $\vartheta_{a}=\theta_{a}-\theta_{R}$ represents the temperature change of particle $a$. Making use of the linear momentum equation (54a), the deformation gradient equation (54b) and the entropy equation (57) enables the above expression after some algebra to yield

$$
\begin{aligned}
\sum_{a} V_{R}^{a} \frac{d B_{a}}{d t}-\dot{\Pi}_{\mathrm{ext}}-\mathcal{Q}_{\mathrm{ext}} & =\sum_{a} \sum_{b \in \Lambda_{a}^{b}} \frac{1}{2}\left[\left(\boldsymbol{P}_{a} \mathbf{C}_{a b}\right) \cdot \boldsymbol{v}_{b}-\left(\boldsymbol{P}_{b} \mathbf{C}_{b a}\right) \cdot \boldsymbol{v}_{a}\right] \\
& +\sum_{a} \sum_{b \in \Lambda_{a}^{b}} \frac{\theta_{R}}{2}\left[\left(\frac{\boldsymbol{Q}_{a}}{\theta_{a}} \cdot \mathbf{C}_{a b}\right)-\left(\frac{\boldsymbol{Q}_{b}}{\theta_{b}} \cdot \mathbf{C}_{b a}\right)\right] \\
& +\sum_{a} V_{R}^{a} \frac{\theta_{R}}{\theta_{a}^{2}} \boldsymbol{Q}_{a} \cdot \nabla_{0} \theta\left(\boldsymbol{X}_{a}, t\right)+\sum_{a} \sum_{b \in \Lambda_{a}^{b}}\left(\boldsymbol{v}_{a} \cdot \mathcal{D}_{\boldsymbol{p}}^{a b}+\Sigma_{J}^{a} \mathcal{D}_{J}^{a b}+\vartheta_{a} \mathcal{D}_{\eta}^{a b}\right)
\end{aligned}
$$

where $\left\{\mathcal{D}_{\boldsymbol{p}}^{a b}, \mathcal{D}_{J}^{a b}, \mathcal{D}_{\eta}^{a b}\right\}$ represent appropriate numerical stabilisation terms, which need not coincide with those used in the case of $E$ being used instead of $\eta$ as the thermal variable. Again, due to the anti-symmetric nature of the terms in the first line and the second line of the right hand side, we can conclude that these terms cancel and thus (68) reduces to

$$
\sum_{a} V_{R}^{a} \frac{d B_{a}}{d t}-\dot{\Pi}_{\mathrm{ext}}-\mathcal{Q}_{\mathrm{ext}}=\sum_{a} V_{R}^{a} \frac{\theta_{R}}{\theta_{a}^{2}} \boldsymbol{Q}_{a} \cdot \nabla_{0} \theta\left(\boldsymbol{X}_{a}, t\right)-\mathcal{D}_{\text {total }}^{\eta}
$$

where

$$
\mathcal{D}_{\text {total }}^{\eta}=\frac{1}{2} \sum_{a} \sum_{b \in \Lambda_{a}^{b}} \mathcal{D}_{\text {total }, a b}^{\eta}
$$


with

$$
\mathcal{D}_{\text {total }, a b}^{\eta}=\left(\boldsymbol{v}_{b}-\boldsymbol{v}_{a}\right) \cdot \mathcal{D}_{\boldsymbol{p}}^{a b}+\left(\Sigma_{J}^{b}-\Sigma_{J}^{a}\right) \mathcal{D}_{J}^{a b}+\left(\vartheta_{b}-\vartheta_{a}\right) \mathcal{D}_{\eta}^{a b}
$$

To arrive at equation (70), we have used a similar reasoning to that in equations (63)-(65) and we have imposed local conservation $\mathcal{D}_{\eta}^{a b}=-\mathcal{D}_{\eta}^{b a}, \mathcal{D}_{\boldsymbol{p}}^{a b}=-\mathcal{D}_{\boldsymbol{p}}^{b a}$ and $\mathcal{D}_{J}^{a b}=-\mathcal{D}_{J}^{b a}$. In above equation $(69)$, the first term on the right hand side involving $\boldsymbol{Q}_{a} \cdot \boldsymbol{\nabla}_{0} \theta\left(\boldsymbol{X}_{a}, t\right) \leq 0$ is non-positive due to the definition of conductive heat flux. It remains to ensure that the second term in the right hand side of $(69) \mathcal{D}_{\text {total }}^{\eta}$ is non-negative. Sufficient conditions guaranteeing this is the case, namely $\mathcal{D}_{\text {total }}^{\eta} \geq 0(70)$, are

$$
\begin{aligned}
& \mathcal{D}_{\boldsymbol{p}}^{a b}=\boldsymbol{S}_{a b}^{\boldsymbol{p}}\left(\boldsymbol{v}_{b}-\boldsymbol{v}_{a}\right) ; \\
& \mathcal{D}_{J}^{a b}=S_{a b}^{J}\left(\Sigma_{J}^{b}-\Sigma_{J}^{a}\right) ; \\
& \mathcal{D}_{\eta}^{a b}=S_{a b}^{\eta}\left(\vartheta_{b}-\vartheta_{a}\right),
\end{aligned}
$$

where $\boldsymbol{S}_{a b}^{p}$ is a positive semi-definite stabilisation matrix and both terms $\left\{S_{a b}^{J}, S_{a b}^{\eta}\right\} \geq 0$. Again, a Riemann solver type of upwinding stabilisation arises.

Remark 6: In this work, the dissipation terms used for the $\eta$ formulation are chosen as

$$
\boldsymbol{S}_{a b}^{p}=\frac{1}{2} \rho_{R, a b}^{\mathrm{Ave}}\left\|\mathbf{C}_{a b}^{\mathrm{Skew}}\right\| \boldsymbol{A}_{a b} ; \quad S_{a b}^{J}=\frac{\mathbf{c}_{a b}^{\mathrm{Skew}} \cdot \mathbf{c}_{a b}^{\mathrm{Skew}}}{2 \rho_{R, a b}^{\mathrm{Ave}} c_{p, a b}^{\mathrm{Ave}}\left\|\mathbf{C}_{a b}^{\text {Skew }}\right\|} ; \quad S_{a b}^{\eta}=0,
$$

where

$$
\boldsymbol{A}_{a b}=c_{p, a b}^{\mathrm{Ave}} \boldsymbol{n}_{a b}^{\mathrm{Skew}} \otimes \boldsymbol{n}_{a b}^{\mathrm{Skew}}+c_{s, a b}^{\mathrm{Ave}}\left(\boldsymbol{I}-\boldsymbol{n}_{a b}^{\mathrm{Skew}} \otimes \boldsymbol{n}_{a b}^{\mathrm{Skew}}\right)
$$

and

$$
\rho_{R, a b}^{\mathrm{Ave}}=\frac{1}{2}\left(\rho_{R, a}+\rho_{R, b}\right) ; \quad c_{p, a b}^{\mathrm{Ave}}=\frac{1}{2}\left(c_{p, a}+c_{p, b}\right) ; \quad c_{s, a b}^{\mathrm{Ave}}=\frac{1}{2}\left(c_{s, a}+c_{s, b}\right),
$$

where $\rho_{R, a}$ denotes the material density evaluated at particle $a$ and $c_{p, a}$ and $c_{s, a}$ represent the volumetric and shear wave speeds (computed at particle $a$ ). In addition, the material vector $\mathbf{C}_{a b}^{\text {Skew }}$ and its push forward equivalent (spatial) vector $\mathbf{c}_{a b}^{\text {Skew }}$ are defined as $\mathbf{C}_{a b}^{\text {Skew }}=\frac{1}{2}\left(\mathbf{C}_{a b}-\mathbf{C}_{b a}\right)$ and $\mathbf{c}_{a b}^{\text {Skew }}=\frac{1}{2}\left(\boldsymbol{H}_{a} \mathbf{C}_{a b}-\boldsymbol{H}_{b} \mathbf{C}_{b a}\right)$ with their magnitudes (norms) defined as $\left\|\mathbf{C}_{a b}^{\text {Skew }}\right\|$ and $\left\|\mathbf{c}_{a b}^{\text {Skew }}\right\|$, respectively, and $\boldsymbol{n}_{a b}^{\text {Skew }}=\frac{\mathbf{c}_{a b}^{\text {Skew }}}{\left\|\mathbf{c}_{a b}^{\text {Skew }}\right\|}$ representing the (upwinding) direction of the stabilisation.

As for the alternative $E$ formulation, $\mathcal{S}_{a b}^{E}$ is chosen in this work so as to ensure that the same amount of numerical stabilisation is introduced by both $\eta$ and $E$ implementations. One possible way to enforce this is by imposing $\mathcal{D}_{\text {total }, a b}^{\eta}$ in (71) to match $\mathcal{D}_{\text {total,ab }}^{E}$ in (65), which permits to deduce the term $\mathcal{S}_{a b}^{E}$ by solving the scalar equation $\mathcal{D}_{\text {total, } a b}^{\eta}=\mathcal{D}_{\text {total, } a b}^{E}$, resulting in

$$
S_{a b}^{E}=\left(\frac{1}{\Xi_{a}}-\frac{1}{\Xi_{b}}\right)^{-2}\left[\mathcal{D}_{\text {total }, a b}^{\eta}-\boldsymbol{S}_{a b}^{p}:\left[\left(\frac{\boldsymbol{v}_{b}}{\Xi_{b}}-\frac{\boldsymbol{v}_{a}}{\Xi_{a}}\right) \otimes\left(\frac{\boldsymbol{v}_{b}}{\Xi_{b}}-\frac{\boldsymbol{v}_{a}}{\Xi_{a}}\right)\right]-S_{a b}^{J}\left(\frac{\Sigma_{J}^{b}}{\Xi_{b}}-\frac{\Sigma_{J}^{a}}{\Xi_{a}}\right)^{2}\right],
$$

with $\boldsymbol{S}_{a b}^{p}$ and $S_{a b}^{J}$ the positive semi-definite tensors already defined in $(73)$.

\section{Temporal discretisation}

Insofar as the mixed-based system $\{\boldsymbol{p}, \boldsymbol{F}, \boldsymbol{H}, J, E\}(4)$ (or its alternative set $\{\boldsymbol{p}, \boldsymbol{F}, \boldsymbol{H}, J, \eta\}$ ) is rather large, it will only be suitable to employ an explicit type of time integrator. In this work, 
an explicit one-step two-stage Total Variation Diminishing Runge-Kutta (TVD-RK) scheme is utilised [1, 2, 43, 58, 59, 61, 63, 68, 69]. This is described by the following time update equations from time step $t^{n}$ to $t^{n+1}$

$$
\begin{aligned}
\mathcal{U}_{a}^{\star} & =\mathcal{U}_{a}^{n}+\Delta t \dot{\mathcal{U}}_{a}^{n}\left(\mathcal{U}_{a}^{n}, t^{n}\right) ; \\
\mathcal{U}_{a}^{\star \star} & =\mathcal{U}_{a}^{\star}+\Delta t \dot{\mathcal{U}}_{a}^{\star}\left(\mathcal{U}_{a}^{\star}, t^{n+1}\right) ; \\
\mathcal{U}_{a}^{n+1} & =\frac{1}{2}\left(\mathcal{U}_{a}^{n}+\mathcal{U}_{a}^{\star \star}\right) .
\end{aligned}
$$

Moreover, the geometry is also updated through the above TVD-RK algorithm [1, 2, 61]. This results in a monolithic time integration procedure where the unknowns $\mathcal{U}$ together with the geometry $\boldsymbol{x}$ are all updated via $(77)$.

The maximum time step $\Delta t:=t^{n+1}-t^{n}$ is governed by a standard Courant-Friedrichs-Lewy $(\mathrm{CFL})$ condition [70] given as

$$
\Delta t=\alpha_{C F L} \frac{h_{\min }}{c_{p, \max }}
$$

where $c_{p, \text { max }}$ is the maximum $p$-wave speed ${ }^{13}, h_{\text {min }}$ is the smallest particle spacing within the computational domain and $\alpha_{C F L}$ is the CFL stability number. For the numerical computations presented in this paper, a value of $\alpha_{C F L}=0.3$, unless otherwise stated, has been chosen to ensure both accuracy and stability [63] of the algorithm.

It is worth mentioning that the resulting SPH algorithm (54) does not intrinsically fulfill conservation of angular momentum, since strong compatibilities between the three strain measures $\{\boldsymbol{F}, \boldsymbol{H}, J\}$ are weakly enforced [61. To address this, , a monolithic discrete angular momentum projection algorithm is presented 14 . Specifically, the local internal nodal force $\boldsymbol{T}_{a}$ described in (55) is suitably modified in order to preserve the total angular momentum, whilst still ensuring the global conservation of linear momentum.

Following reference [1, sufficient conditions for the global preservation of the discrete linear and angular momentum within a time step are enforced at each stage of the TVD-RK time integrator (77) described as

$$
\sum_{a} V_{R}^{a} \boldsymbol{T}_{a}^{\chi}=\mathbf{0} ; \quad \sum_{a} V_{R}^{a} \mathscr{X}_{a} \times \boldsymbol{T}_{a}^{\chi}=\mathbf{0} ; \quad \mathscr{X}_{a}=\left\{\begin{array}{cl}
\boldsymbol{x}_{a}^{n}, & \chi=n \\
\boldsymbol{x}_{a}^{n}+\frac{\Delta t}{2 \rho_{R}}\left(\boldsymbol{p}_{a}^{n}+\boldsymbol{p}_{a}^{*}\right), & \chi=*
\end{array}\right.
$$

where $\chi=\{n, *\}$.

A least-square minimisation procedure is used to obtain a set of modified internal nodal forces $\hat{\boldsymbol{T}}_{a}$ that satisfy the above conditions $(79)$. This can be achieved by computing the minimum of the following functional (ignoring time arguments for brevity), that is

$$
\Pi\left(\hat{\boldsymbol{T}}_{a}, \boldsymbol{\lambda}_{\text {ang }}, \boldsymbol{\lambda}_{\text {lin }}\right)=\frac{1}{2} \sum_{a} V_{R}^{a}\left(\hat{\boldsymbol{T}}_{a}-\boldsymbol{T}_{a}\right) \cdot\left(\hat{\boldsymbol{T}}_{a}-\boldsymbol{T}_{a}\right)-\boldsymbol{\lambda}_{\text {ang }} \cdot\left(\sum_{a} V_{R}^{a} \mathscr{X}_{a} \times \hat{\boldsymbol{T}}_{a}\right)-\boldsymbol{\lambda}_{\operatorname{lin}} \cdot\left(\sum_{a} V_{R}^{a} \hat{\boldsymbol{T}}_{a}\right) .
$$

\footnotetext{
${ }^{13}$ The reader is referred to [50] for an accurate computation of this wave speed for the two constitutive models presented in Section 2 of this paper.

${ }^{14}$ In our experience, the angular momentum projection algorithm is only needed for problems involving very large and sustained rotations.
} 
After some simple algebra, a set of modified internal nodal forces $\hat{\boldsymbol{T}}_{a}$ arises

$$
\hat{\boldsymbol{T}}_{a}=\boldsymbol{T}_{a}+\boldsymbol{\lambda}_{\text {ang }} \times \mathscr{X}_{a}+\boldsymbol{\lambda}_{\text {lin }}
$$

for which the Lagrange multipliers $\left\{\boldsymbol{\lambda}_{\text {ang }}, \boldsymbol{\lambda}_{\text {lin }}\right\}$ are the solutions of the following system of equations

$$
\left[\begin{array}{cc}
\sum_{a} V_{R}^{a}\left[\left(\mathscr{X}_{a} \cdot \mathscr{X}_{a}\right) \boldsymbol{I}-\mathscr{X}_{a} \otimes \mathscr{X}_{a}\right] & \sum_{a} V_{R}^{a} \hat{\mathscr{X}}_{a} \\
\sum_{a} V_{R}^{a} \hat{\mathscr{X}}_{a} & -\sum_{a} V_{R}^{a}
\end{array}\right]\left[\begin{array}{c}
\boldsymbol{\lambda}_{\text {ang }} \\
\boldsymbol{\lambda}_{\operatorname{lin}}
\end{array}\right]=\left[\begin{array}{c}
-\sum_{a} V_{R}^{a} \mathscr{X}_{a} \times \boldsymbol{T}_{a} \\
\sum_{a} V_{R}^{a} \boldsymbol{T}_{a}
\end{array}\right],
$$

where the indicial notation $\left[\hat{\mathscr{X}}_{a}\right]_{i k}=\mathcal{E}_{i j k}\left[\mathscr{X}_{a}\right]_{j}$.

\section{Algorithmic description}

For ease of implementation, Algorithm 1 summarises the complete algorithmic description of the mixed-based Upwind Smooth Particle Hydrodynamics (Upwind-SPH) methodology described above.

Algorithm 1: Complete Upwind-SPH algorithm for thermo-elasticity

Input $: \mathcal{U}_{a}^{n} \quad$ where $\mathcal{U}$ either $\left[\begin{array}{lllll}\boldsymbol{p} & \boldsymbol{F} & \boldsymbol{H} & J & E\end{array}\right]^{T}$ or $\left[\begin{array}{lllll}\boldsymbol{p} & \boldsymbol{F} & \boldsymbol{H} & J & \eta\end{array}\right]^{T}$

Output: $\mathcal{U}_{a}^{n+1}, \boldsymbol{P}_{a}^{n+1}, \theta_{a}^{n+1}, \boldsymbol{x}_{a}^{n+1}$

(1) ASSIGN old primary variables: $\mathcal{U}_{a}^{\text {old }}=\mathcal{U}_{a}^{n}$ and $\boldsymbol{x}_{a}^{\text {old }}=\boldsymbol{x}_{a}^{n}$

(2) EVALUATE $p$-wave speed: $c_{p}$ (see References [61, 65])

(3) COMPUTE time increment: $\Delta t$

for $T V D-R K$ time integrator $=1$ to 2 do

(4) COMPUTE right-hand-side of the mixed-based system: $\dot{\boldsymbol{p}}_{a}$ 54a), $\dot{\boldsymbol{F}}_{a}$ 54b, $\dot{\boldsymbol{H}}_{a}$ 54c), $\dot{J}_{a}$ (54d) and $\dot{E}_{a}$ (54e) (or $\dot{\eta}_{a}$ (57)

(5) APPLY discrete angular momentum preserving algorithm (see Section 6 of [1])

(6) EVOLVE $\left\{\mathcal{U}_{a}, \boldsymbol{x}_{a}\right\}$ via TVD-RK (77)

(7) IMPOSE essential boundary conditions directly on particles $\boldsymbol{p}_{a}$ and $E_{a}$ (or $\eta_{a}$ )

(8) COMPUTE first Piola-Kirchhoff stress tensor $\boldsymbol{P}_{a}$

(9) COMPUTE temperature $\theta_{a}$

end

(10) UPDATE $\left\{\boldsymbol{U}_{a}^{n+1}, \boldsymbol{x}_{a}^{n+1}\right\}$

(11) COMPUTE first Piola-Kirchhoff stress tensor $\boldsymbol{P}_{a}^{n+1}$

(12) COMPUTE temperature $\theta_{a}^{n+1}$

\section{Numerical examples}

In this section, a number of challenging numerical examples are presented in order to assess the performance of the Upwind Smooth Particle Hydrodynamics (Upwind-SPH) described above. The examples will be used to demonstrate that the Upwind-SPH algorithm: 
- achieves second order convergence for all the variables solved, namely velocities, stresses (or strains) and temperature (or entropy or total energy),

- circumvents spurious zero-energy modes due to rank deficiency,

- removes non-physical pressure instabilities when approaching the near incompressibility limit,

- preserves total angular momentum and total energy over a long term response, and

- guarantees long-term stability by ensuring a non-negative rate of production of entropy (when considering an isolated system) and, simultaneously, displays a decrease of the ballistic energy.

For all the examples presented in this paper, uniform particle refinement is employed. Additionally, the constant smoothing length is determined by multiplying the largest particle spacing within the compact support with a scale factor of 0.6. Notice that for the case of non-smooth (i.e. shock dominated) problems, which rests outside the scope of this paper, the use of non-uniform particle refinement should be considered [71 73].

It will be shown that both proposed formulations, namely $\{\boldsymbol{p}, \boldsymbol{F}, \boldsymbol{H}, J, E\}$ and $\{\boldsymbol{p}, \boldsymbol{F}, \boldsymbol{H}, J, \eta\}$, yield extremely similar results, almost undistinguishable. In addition, and for benchmarking purposes, the SPH discretisation will also be compared against an alternative in-house Vertex Centred Finite Volume implementation.

The material properties used in the examples are typically given in terms of the linear thermal expansion coefficient $\alpha$, the Young's modulus $E$ and the Poisson's ratio $\nu$, from which the shear and Bulk moduli can be obtained as

$$
\mu=\frac{E}{2(1+\nu)} ; \quad \kappa=\frac{E}{3(1-2 \nu)},
$$

which permit to calibrate the material parameters according to equations (29) and (32).

\subsection{Manufactured problem: linear thermo-elasticity}

The main objective of this example is to display the spatial convergence of the proposed $\{\boldsymbol{p}, \boldsymbol{F}, \boldsymbol{H}, J, E\}$ Upwind-SPH methodology. Inspired by Reference [50], a cube of unit side length $L=1 \mathrm{~m}$ (see Figure 2) is subjected to a thermo-mechanical deformation process given by the following mapping and temperature functions

$$
\begin{aligned}
& \boldsymbol{\phi}^{\text {exact }}(\boldsymbol{X}, t)=\boldsymbol{X}+\frac{A t}{L}(\boldsymbol{X} \cdot \boldsymbol{X}) \mathbf{1} \\
& \theta^{\text {exact }}(\boldsymbol{X}, t)=\theta_{R}\left[1+B \sin \left(\frac{\pi \boldsymbol{X} \cdot \boldsymbol{E}_{1}}{L}\right)(t+1)\right],
\end{aligned}
$$

where $\mathbf{1}=[1,1,1]^{T}$ and $\boldsymbol{E}_{1}=[1,0,0]^{T}$. For a range of $A$ and $B$ values, the solution can be considered to be linear and a closed-form solution can be derived. In this case, the values of $A=0.2381 \mathrm{~s}^{-1}$ and $B=-0.0012 \mathrm{~s}^{-1}$ are used. A linear thermo-elastic model is employed with Young's modulus $E=50.05 \mathrm{KPa}$, density $\rho_{R}=1000 \mathrm{~kg} / \mathrm{m}^{3}$, Poisson's ratio $\nu=0.3$, thermal conductivity $h=10 \mathrm{Wm}^{-1} \mathrm{~K}^{-1}$, specific heat capacity $C_{v}=1 \mathrm{Jkg}^{-1} \mathrm{~K}^{-1}$, thermal expansion coefficient $\alpha=2.223 \times 10^{-4} \mathrm{~K}^{-1}$ and reference temperature $\theta_{R}=293.15 \mathrm{~K}$. 
The exact velocity $\boldsymbol{v}^{\text {exact }}(\boldsymbol{X}, t)$, the exact deformation gradient tensor $\boldsymbol{F}^{\text {exact }}(\boldsymbol{X}, t)$ and the exact temperature gradient $\boldsymbol{\nabla}_{0} \theta^{\text {exact }}(\boldsymbol{X}, t)$ follow from (84) as

$$
\begin{gathered}
\boldsymbol{v}^{\text {exact }}(\boldsymbol{X}, t)=\frac{\partial \phi^{\text {exact }}(\boldsymbol{X}, t)}{\partial t}=\frac{A}{L}(\boldsymbol{X} \cdot \boldsymbol{X}) \mathbf{1}, \\
\boldsymbol{F}^{\text {exact }}(\boldsymbol{X}, t)=\boldsymbol{\nabla}_{0} \boldsymbol{\phi}^{\text {exact }}(\boldsymbol{X}, t)=\boldsymbol{I}+\frac{2 A t}{L} \mathbf{1} \otimes \boldsymbol{X}, \\
\boldsymbol{\nabla}_{0} \theta^{\text {exact }}(\boldsymbol{X}, t)=\left(\frac{B \pi \theta_{R}(t+1)}{L}\right) \cos \left(\frac{\pi \boldsymbol{X} \cdot \boldsymbol{E}_{1}}{L}\right) \boldsymbol{E}_{1} .
\end{gathered}
$$

This enables the evaluation of the area and volume mappings as [58]

$$
\boldsymbol{H}^{\text {exact }}(\boldsymbol{X}, t)=\frac{1}{2} \boldsymbol{F}^{\text {exact }} \times \boldsymbol{F}^{\text {exact }} ; \quad J^{\text {exact }}(\boldsymbol{X}, t)=\frac{1}{3} \boldsymbol{H}^{\text {exact }}: \boldsymbol{F}^{\text {exact }} .
$$

With these at hand, we can obtain the exact expressions for the body force $\boldsymbol{f}_{R}(1 \mathrm{a})$ and the heat source term $s_{R}(14)$. Using the chain rule, and after some simple algebra, the body force term becomes

$$
\boldsymbol{f}_{R}=\frac{\partial\left(\rho_{R} \boldsymbol{v}^{\text {exact }}\right)}{\partial t}-\operatorname{DIV} \boldsymbol{P}^{\text {exact }}=-(2 \mu+\lambda)\left(\frac{2 A t}{L}\right) \mathbf{1}+\frac{B C_{v} \Gamma_{0} \theta_{R} \pi}{L} \cos \left(\frac{\pi \boldsymbol{X} \cdot \boldsymbol{E}_{1}}{L}\right)(t+1) \boldsymbol{E}_{1}
$$

and the heat source term yields

$$
s_{R}=\theta^{\text {exact }}\left[B C_{v} \sin \left(\frac{\pi \boldsymbol{X} \cdot \boldsymbol{E}_{1}}{L}\right)+\frac{2 A C_{v} \Gamma_{0}}{L}(\boldsymbol{X} \cdot \mathbf{1})\right]+B h \theta_{R}\left(\frac{\pi}{L}\right)^{2}(t+1) \sin \left(\frac{\pi \boldsymbol{X} \cdot \boldsymbol{E}_{1}}{L}\right) .
$$

Dirichlet boundary conditions compatible with the exact fields $\phi^{\text {exact }} 84 \mathrm{a}$ and $\theta^{\text {exact }} 84 \mathrm{~b}$ are applied on the boundary of the domain. Specifically, fixed (i.e. restricted the movement in all directions) boundary conditions are enforced at position $X=Y=Z=0$. Moreover, fixed reference temperature $\theta=\theta_{R}$ is constantly applied on the faces $X=0$ and $X=1$.

Additionally, initial conditions for the velocity, the triplet of deformation measures and temperature (see Figure 2) can be obtained by substituting time $t=0$ into expressions (85), (86), (88) and 84b, which results in

$$
\begin{aligned}
& \left.\boldsymbol{v}\right|_{t=0}=\boldsymbol{v}^{\text {exact }}(\boldsymbol{X}, t=0)=\frac{A}{L}(\boldsymbol{X} \cdot \boldsymbol{X}) \mathbf{1} \\
& \left.\boldsymbol{F}\right|_{t=0}=\boldsymbol{F}^{\text {exact }}(\boldsymbol{X}, t=0)=\boldsymbol{I} ;\left.\quad \boldsymbol{H}\right|_{t=0}=\boldsymbol{H}^{\text {exact }}(\boldsymbol{X}, t=0)=\boldsymbol{I} ;\left.\quad J\right|_{t=0}=J^{\text {exact }}(\boldsymbol{X}, t=0)=1 \\
& \left.\theta\right|_{t=0}=\theta^{\text {exact }}(\boldsymbol{X}, t=0)=\theta_{R}\left(1+B \sin \left(\frac{\pi \boldsymbol{X} \cdot \boldsymbol{E}_{1}}{L}\right)\right)
\end{aligned}
$$

Figure 3 displays the spatial order of convergence of the numerical SPH approximation against the closed-form solution. For completeness, the spatial convergence of the numerical approximation using an in-house Upwind Vertex Centred Finite Volume Method (UpwindVCFVM) [43] is also depicted. Both SPH and vertex centred implementations use the same 


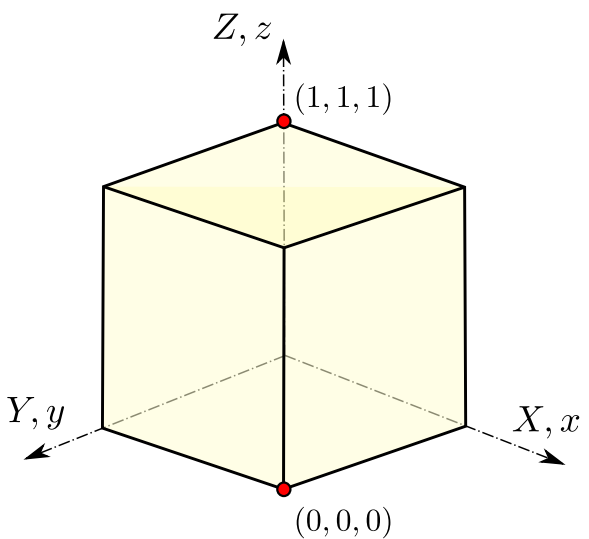

(a)

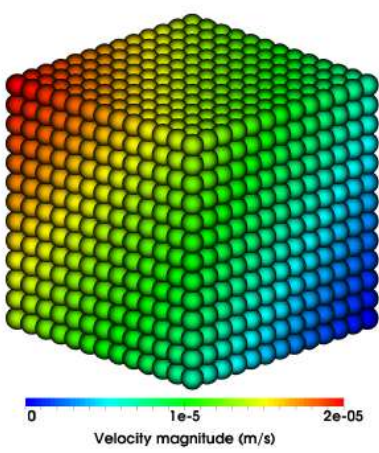

(b)

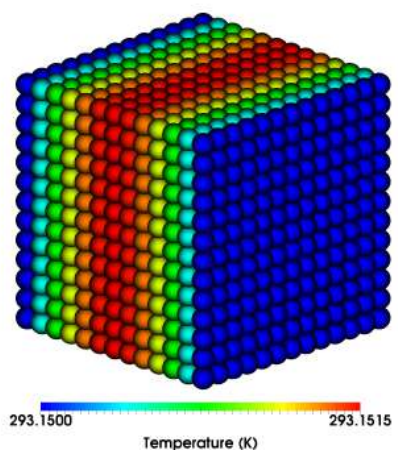

(c)

Figure 2: Linear thermo-elasticity: (a) geometry and the initial conditions for (b) velocity and (c) temperature.

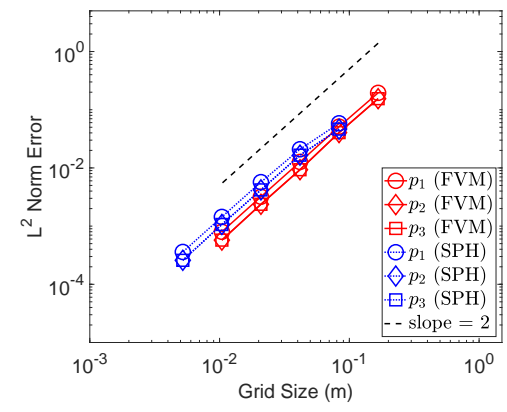

(a)

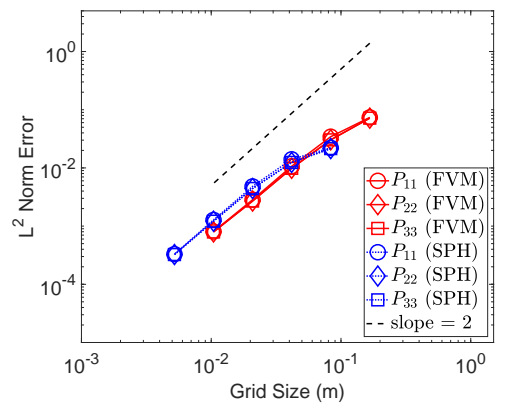

(b)

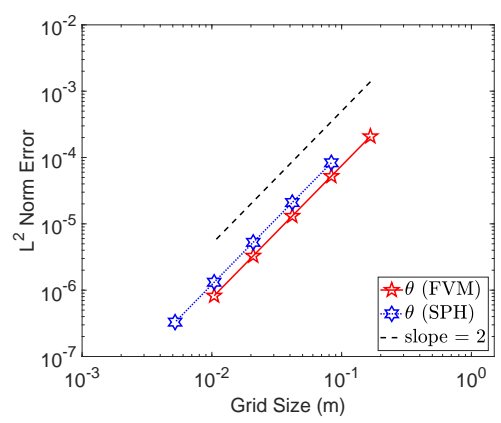

(c)

Figure 3: Linear thermo-elasticity: $L_{2}$ norm convergence of (a) the components of linear momentum; (b) the components of the first Piola-Kirchhoff stress; and (c) temperature at a particular time $t=0.001$ s. Results obtained using a linear thermo-elastic model. The material properties used are Young's modulus $E=50.05 \mathrm{KPa}$, density $\rho_{R}=1000 \mathrm{~kg} / \mathrm{m}^{3}$, thermal conductivity $h=10 \mathrm{Wm}^{-1} \mathrm{~K}^{-1}$, Specific heat capacity $C_{v}=1 \mathrm{Jkg}^{-1} \mathrm{~K}^{-1}$, thermal expansion coefficient $\alpha=2.223 \times 10^{-4} \mathrm{~K}^{-1}$ and $\alpha_{\mathrm{CFL}}=0.3$. Grid size refers to the minimum particle spacing between particles within the computational domain. 


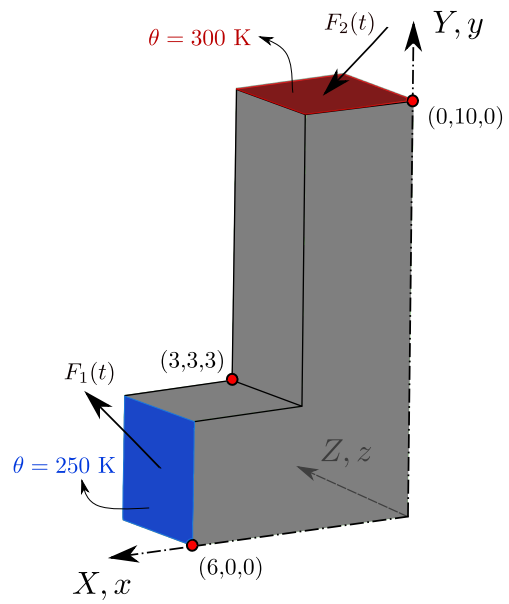

Figure 4: L-shaped block configuration

Riemann based numerical dissipation. In all cases, Figure 3 depicts the expected second order convergence pattern (e.g. $L^{2}$ norm error) for the components of the linear momentum, components of the stress tensor (same results are obtained for all the components of the stress tensor) and temperature. It is interesting to note that the proposed Upwind-SPH methodology requires a finer grid size (i.e. the characteristic length defining the minimum spacing between particles) for the same level of accuracy than Upwind-VCFVM [43]. This is expected as the SPH algorithm is prone to a slightly more diffusive behaviour, due to the use of SPH interpolating functions.

\subsection{L-shaped block}

As reported in References [44, 46, 50, 74, 75], a flexible L-shape block structure is studied in this section. The main objective of this problem is to examine the capability of the proposed $\{\boldsymbol{p}, \boldsymbol{F}, \boldsymbol{H}, J, E\}$ Upwind-SPH algorithm in preserving linear momentum, angular momentum and also the total energy over a long term response. The structure is subjected to time-varying forces (see Figure 4) on two of its boundary faces and is then left free flying in space. From the thermal viewpoint, all boundaries are treated as insulated, thus with null normal heat flux across. The applied forces are described as

$$
\boldsymbol{F}_{1}(t)=-\boldsymbol{F}_{2}(t)=\left[\begin{array}{c}
150 \\
300 \\
450
\end{array}\right] f(t) ; \quad f(t)= \begin{cases}t & 0 \leq t<2.5 \mathrm{~s} \\
5-t & 2.5 \mathrm{~s} \leq t<5 \mathrm{~s} \\
0 & t \geq 5 \mathrm{~s}\end{cases}
$$

The temperature profile across the structure is initiated by

$$
\left.\theta\right|_{t=0}= \begin{cases}300 \mathrm{~K} & Y=10 \mathrm{~m} \\ 250 \mathrm{~K} & X=6 \mathrm{~m} \\ \theta_{R} & \text { elsewhere. }\end{cases}
$$

In this case, a polyconvex Mie-Grüneisen equation of state is employed with Young's modulus $E=50.05 \mathrm{KPa}$, density $\rho_{R}=1000 \mathrm{~kg} / \mathrm{m}^{3}$, Poisson's ratio $\nu=0.3$, thermal conductivity $h=10 \mathrm{Wm}^{-1} \mathrm{~K}^{-1}$, specific heat capacity $C_{v}=1 \mathrm{Jkg}^{-1} \mathrm{~K}^{-1}$ thermal expansion coefficient $\alpha=2.223 \times 10^{-4} \mathrm{~K}^{-1}$ and the reference temperature given by $\theta_{R}=293.15 \mathrm{~K}$. 


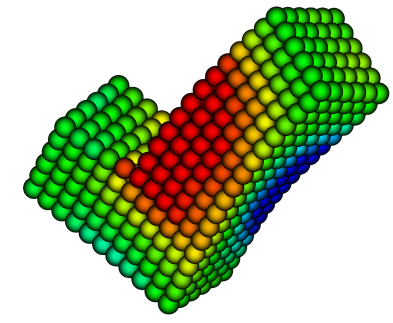

(a) 680 particles

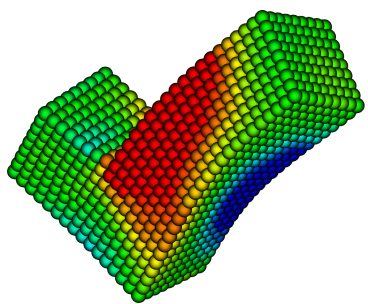

(b) 1900 particles

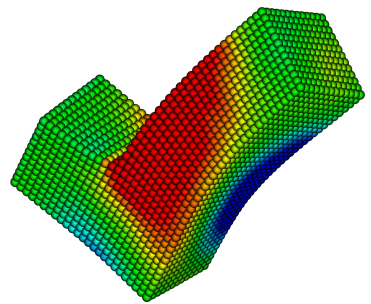

(c) 5400 particles

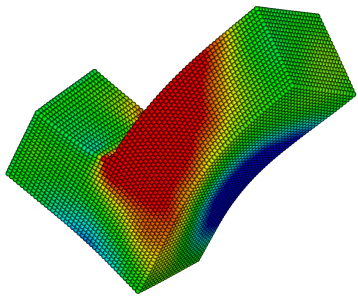

(d) 17600 particles

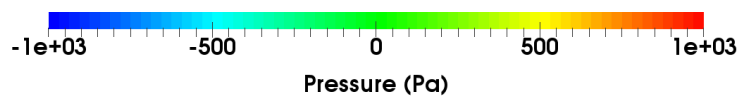

Figure 5: L-shaped block: particle refinement of deformed states with pressure contour plots at time $t=7.7 \mathrm{~s}$ for four different number of particles (a) 680 particles; (b) 1900 particles; (c) 5400 particles and (d) 17600 particles. Results obtained using a polyconvex Mie-Grüneisen equation of state. The material properties are Young's modulus $E=50.05 \mathrm{KPa}$, density $\rho_{R}=1000 \mathrm{~kg} / \mathrm{m}^{3}$, Poisson's ratio $\nu=0.3$, thermal conductivity $h=10$ $\mathrm{Wm}^{-1} \mathrm{~K}^{-1}$, specific heat capacity $C_{v}=1 \mathrm{Jkg}^{-1} \mathrm{~K}^{-1}$, thermal expansion coefficient $\alpha=2.223 \times 10^{-4} \mathrm{~K}^{-1}$, the reference temperature $\theta_{R}=293.15 \mathrm{~K}$ and $\alpha_{\mathrm{CFL}}=0.3$.
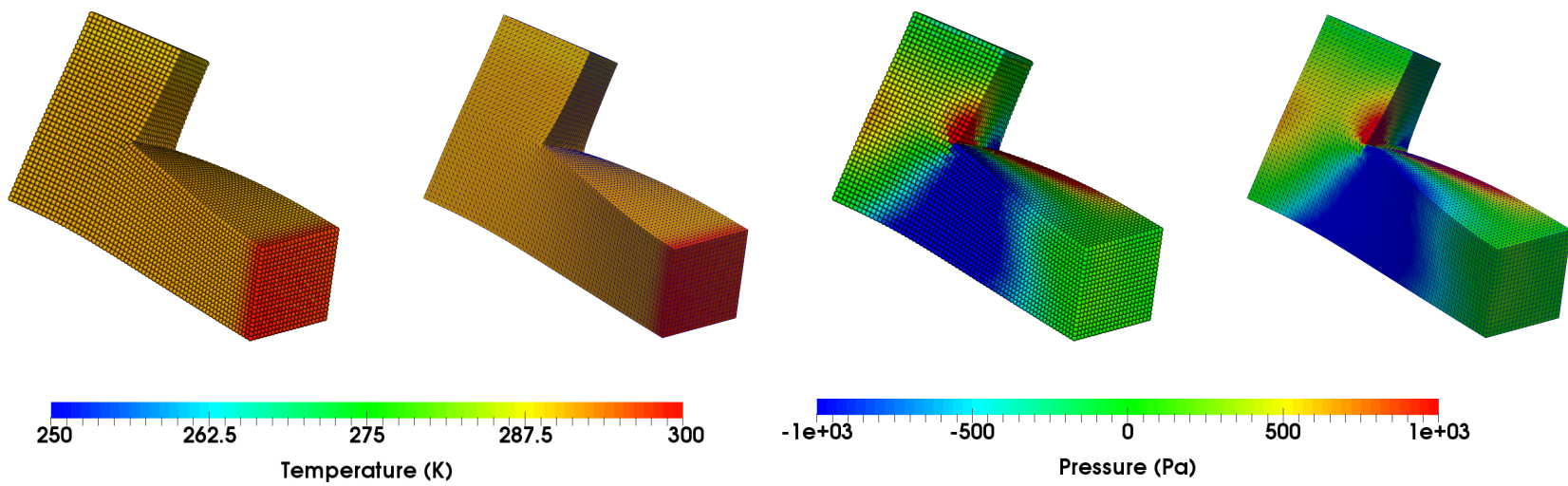

Figure 6: L-shaped block: comparison of deformed shapes plotted with pressure and temperature distributions using the Upwind-SPH and Upwind-VCFVM at time $t=11 \mathrm{~s}$. Results obtained using a polyconvex MieGrüneisen equation of state with material properties Young's modulus $E=50.05 \mathrm{KPa}$, density $\rho_{R}=1000$ $\mathrm{kg} / \mathrm{m}^{3}$, Poisson's ratio $\nu=0.3$, thermal conductivity $h=10 \mathrm{Wm}^{-1} \mathrm{~K}^{-1}$, specific heat capacity $C_{v}=1$ $\mathrm{Jkg}^{-1} \mathrm{~K}^{-1}$, thermal expansion coefficient $\alpha=2.223 \times 10^{-4} \mathrm{~K}^{-1}$, the reference temperature $\theta_{R}=293.15 \mathrm{~K}$ and $\alpha_{\mathrm{CFL}}=0.3$. 


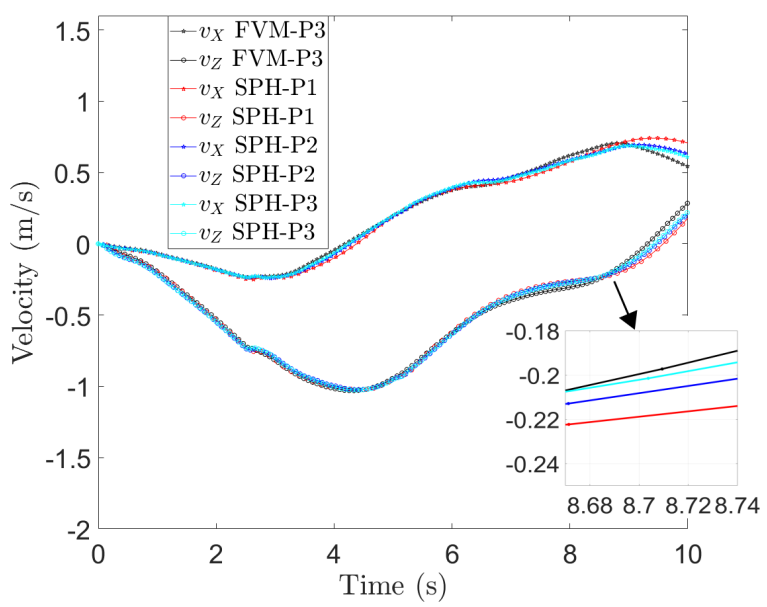

(a)

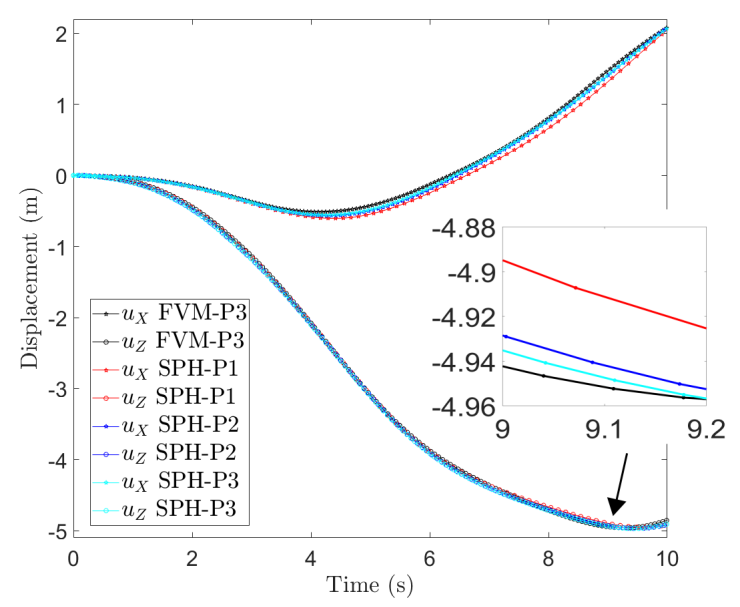

(b)

Figure 7: L-shaped block: time evolution of (a) components of velocity and (b) components of displacement at material point $\boldsymbol{X}=[0,10,0]^{T} \mathrm{~m}$. A comparison is carried out between the $\{\boldsymbol{p}, \boldsymbol{F}, \boldsymbol{H}, J, E\}$ UpwindVCFVM algorithm and the $\{\boldsymbol{p}, \boldsymbol{F}, \boldsymbol{H}, J, E\}$ Upwind-SPH methodology. A polyconvex Mie-Grüneisen equation of state with properties Young's modulus $E=50.05 \mathrm{KPa}$, density $\rho_{R}=1000 \mathrm{~kg} / \mathrm{m}^{3}$, Poisson's ratio $\nu=0.3$, thermal conductivity $h=10 \mathrm{Wm}^{-1} \mathrm{~K}^{-1}$, specific heat capacity $C_{v}=1 \mathrm{Jkg}^{-1} \mathrm{~K}^{-1}$, thermal expansion coefficient $\alpha=2.223 \times 10^{-4} \mathrm{~K}^{-1}$, reference temperature $\theta_{R}=293.15 \mathrm{~K}$ and $\alpha_{\mathrm{CFL}}=0.3 . \mathrm{P} 1, \mathrm{P} 2$ and P3 represent 1900, 5400 and 17600 number of particles/nodes.

A mesh refinement study for the block is carried out in Figure 5 at time $t=7.7 \mathrm{~s}$. For such a sequence of particle refinement, the solver delivers linear computational cost in terms of CPU time with respect to the number of unknowns. Very good predictions for the deformed shape of the structure and the pressure distribution are seen even with the coarsest discretisation. For benchmarking purposes, results are compared against an alternative in-house Upwind-VCFVM [43]. Comparison of the Upwind-SPH and Upwind-VCFVM schemes (see Figure 6) renders almost identical results. The time history of the components of velocity and displacement at position $\boldsymbol{X}=[0,10,0]^{T}$ are also monitored in Figure 7 for completeness.

Figures 8 a,b demonstrate the ability of the proposed method in preserving both the linear and angular momenta of the system. Specifically, the total linear momentum is close to (and oscillates around) zero machine accuracy at all times, whereas the total angular momentum is expected to be conserved after the loading phase, that is when time $t \geq 5 \mathrm{~s}$. In addition, Figure 8c displays the time history of various energetic contributions. These include kinetic energy, internal energy, total energy and ballistic energy. As a result of the external boundary traction, the total energy and the ballistic energy increase during the loading phase. Subsequently, upon the release of the boundary force, that is when $t \geq 5 \mathrm{~s}$, the total energy is expected to remain constant and the ballistic energy is expected to decrease. The difference between the total energy and the Ballistic energy corresponds to the term $\theta_{R} \eta$ which is intimately linked to the global entropy production associated with (i) the irreversible heat conduction and (ii) the generation of numerical entropy due to the numerical scheme itself. This is also shown in Figure $8 \mathrm{~d}$ as the value of global entropy increases over time for the entire simulation.

Finally, Figure 9 illustrates the time evolution of the deformation of the L-shaped block, displaying smooth temperature and pressure fields. It is interesting to note how the heat flows through the insulated body as the result of the initial temperature gradient. 


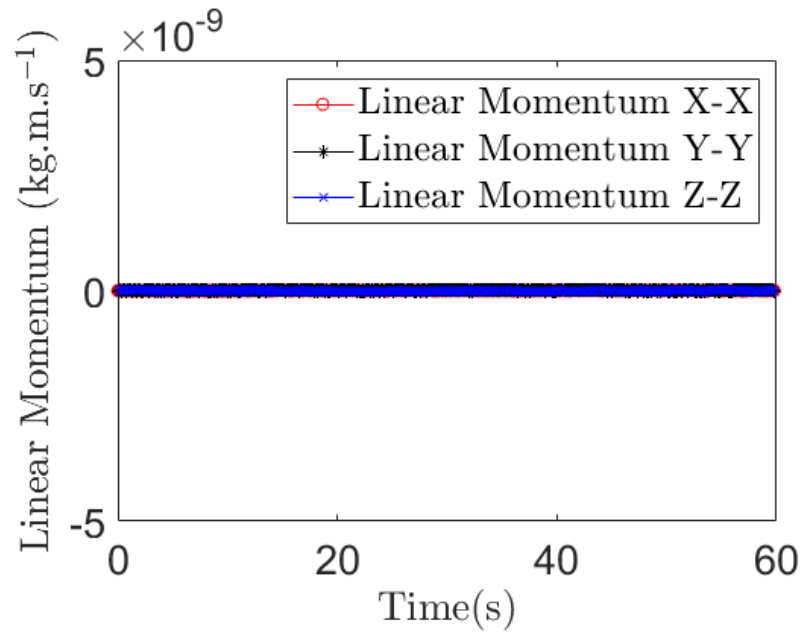

(a)

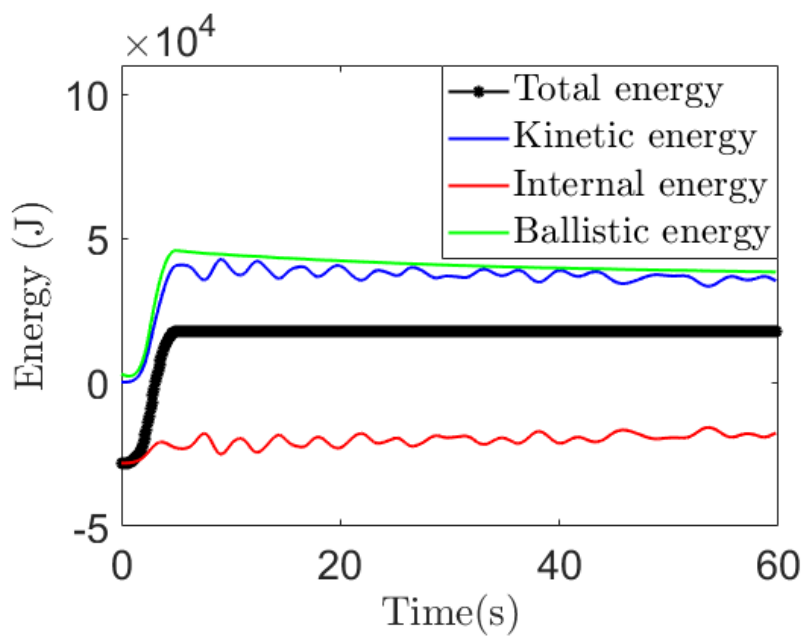

(c)

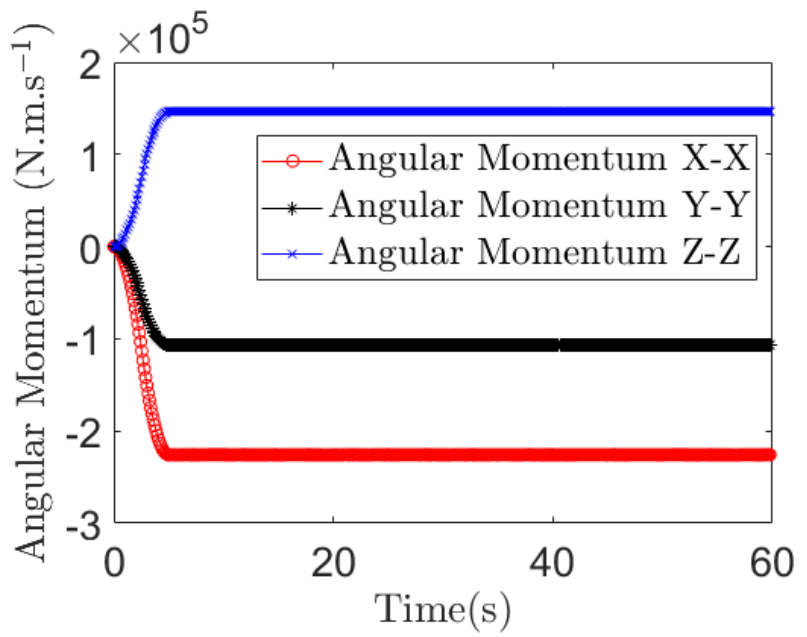

(b)

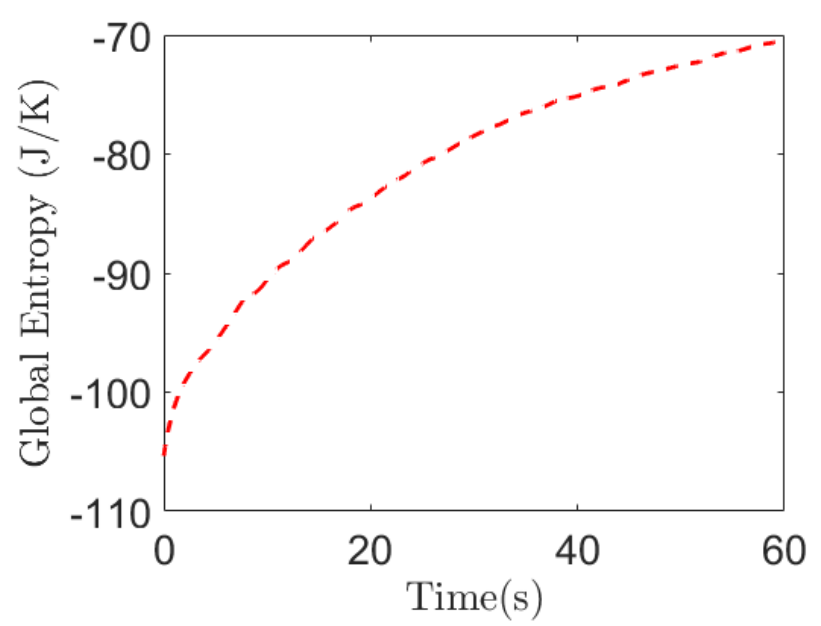

(d)

Figure 8: L-shaped block: time evolution of (a) linear momentum; (b) angular momentum (c) energy measures; and (d) global entropy. Results obtained using a polyconvex Mie-Grüneisen equation of state. The material properties used are Young's modulus $E=50.05 \mathrm{KPa}$, density $\rho_{R}=1000 \mathrm{~kg} / \mathrm{m}^{3}$, Poisson's ratio $\nu=0.3$, thermal conductivity $h=10 \mathrm{Wm}^{-1} \mathrm{~K}^{-1}$, specific heat capacity $C_{v}=1 \mathrm{Jkg}^{-1} \mathrm{~K}^{-1}$, thermal expansion coefficient $\alpha=2.223 \times 10^{-4} \mathrm{~K}^{-1}$, the reference temperature $\theta_{R}=293.15 \mathrm{~K}$ and $\alpha_{\mathrm{CFL}}=0.3$. 

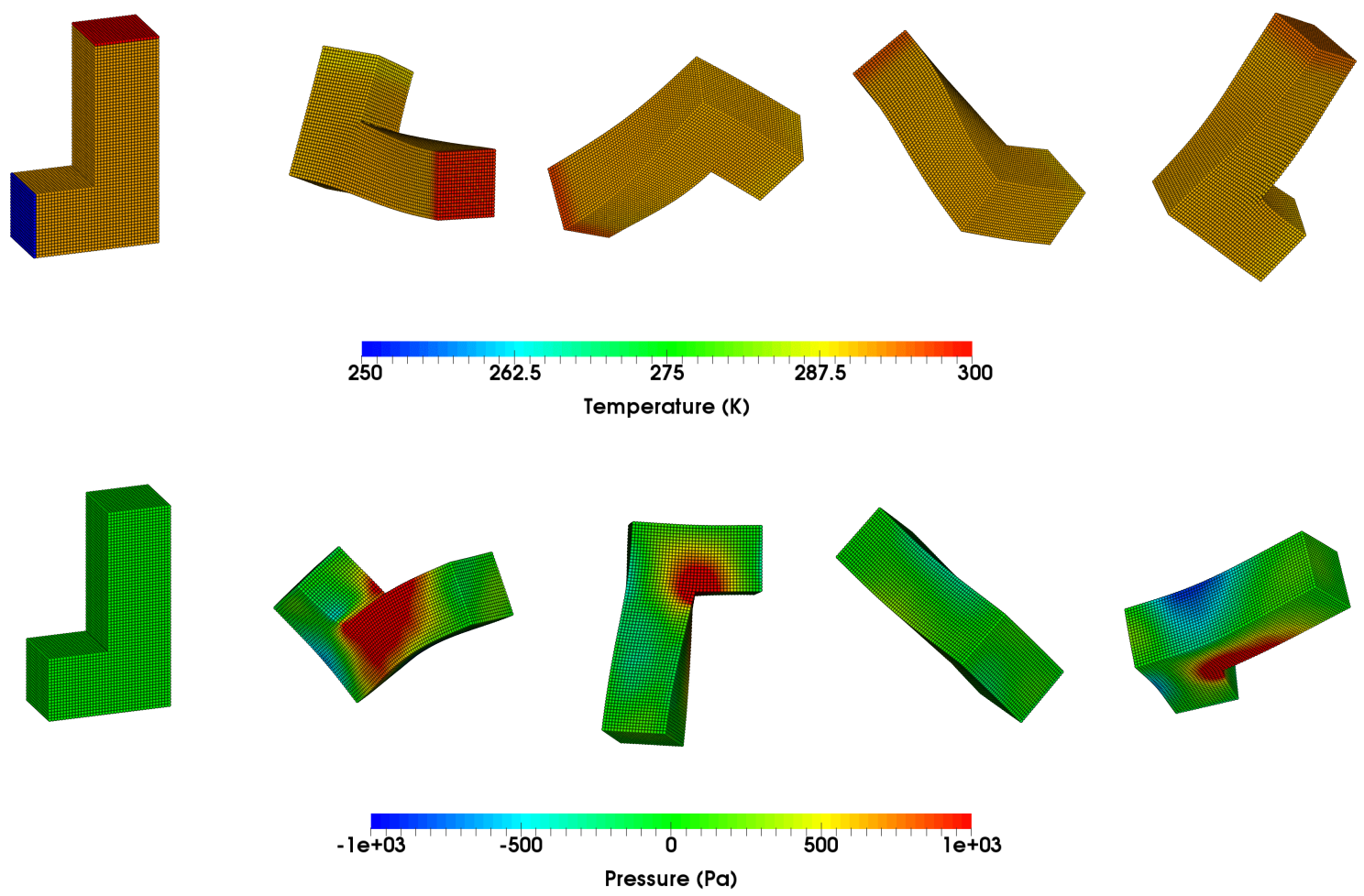

Figure 9: L-shaped block: a sequence of deformed configurations with temperature and pressure distributions at times $t=10 *\{0,1,2,2.5,3\}$ s (left to right) and $t=2 *\{0,4,9,14,19\} \mathrm{s}$ (left to right), respectively. Results obtained using a polyconvex Mie-Grüneisen equation of state. The material properties used are Young's modulus $E=50.05 \mathrm{KPa}$, density $\rho_{R}=1000 \mathrm{~kg} / \mathrm{m}^{3}$, Poisson's ratio $\nu=0.3$, thermal conductivity $h=10 \mathrm{Wm}^{-1} \mathrm{~K}^{-1}$, specific heat capacity $C_{v}=1 \mathrm{Jkg}^{-1} \mathrm{~K}^{-1}$, thermal expansion coefficient $\alpha=2.223 \times 10^{-4} \mathrm{~K}^{-1}$, the reference temperature $\theta_{R}=293.15 \mathrm{~K}$ and $\alpha_{\mathrm{CFL}}=0.3$.

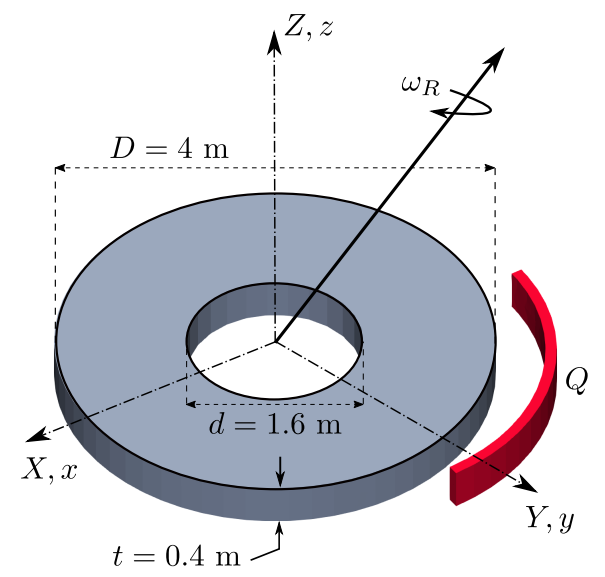

Figure 10: Rotating disk configuration 


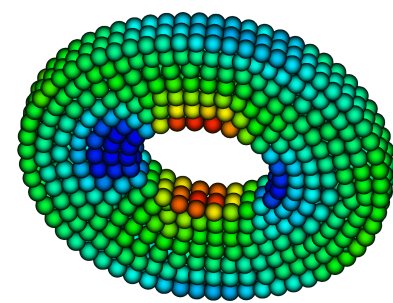

(a) 850 particles

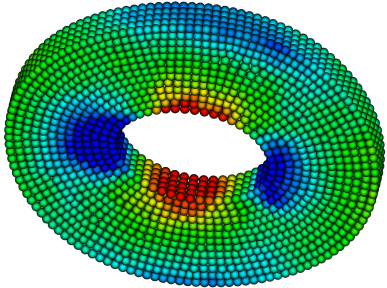

(b) 4500 particles

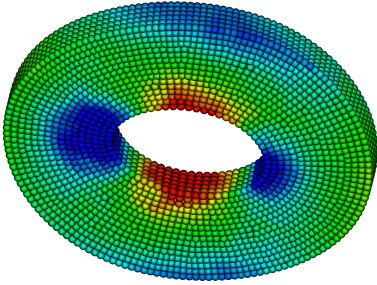

(c) 7800 particles

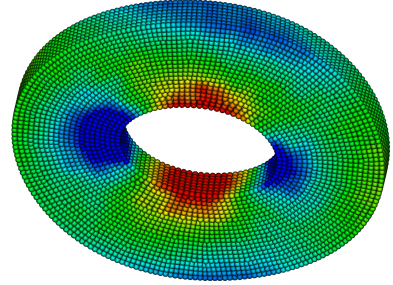

(d) 16000 particles

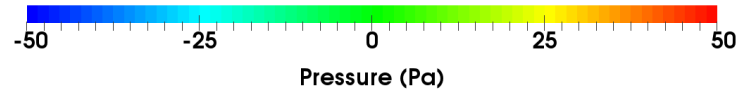

Figure 11: Rotating disk: particle refinement of deformed states with pressure contour plots at time $t=1.35 \mathrm{~s}$ for four different number of particles (a) 850 particles; (b) 4500 particles; (c) 7800 particles and (d) 16000 particles. Results obtained using a polyconvex Mie-Grüneisen equation of state. The material properties used are Young's modulus $E=1474 \mathrm{~Pa}$, density $\rho_{R}=10 \mathrm{~kg} / \mathrm{m}^{3}$, Poisson's ratio $\nu=0.478$, thermal conductivity $h=10$ $\mathrm{Wm}^{-1} \mathrm{~K}^{-1}$, specific heat capacity $C_{v}=10 \mathrm{Jkg}^{-1} \mathrm{~K}^{-1}$, thermal expansion coefficient $\alpha=2.223 \times 10^{-4} \mathrm{~K}^{-1}$, the reference temperature $\theta_{R}=308.15 \mathrm{~K}$ and $\alpha_{\mathrm{CFL}}=0.3$.

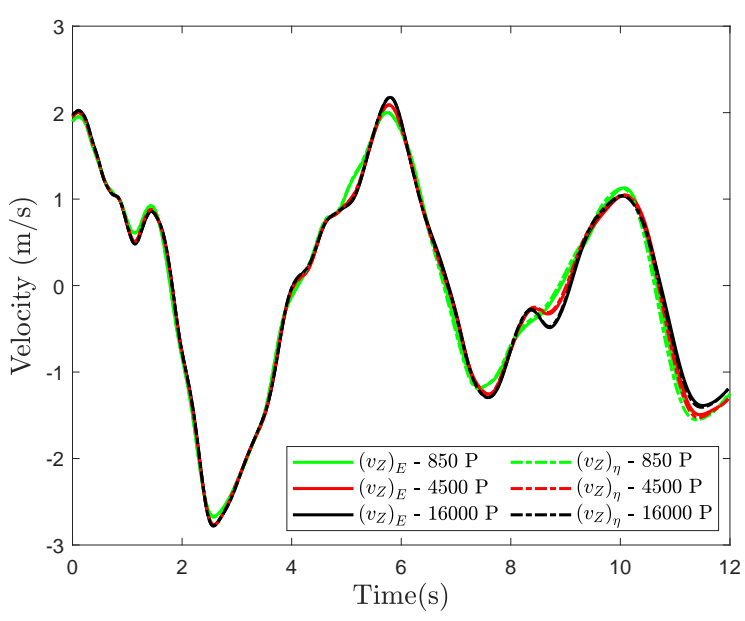

(a)

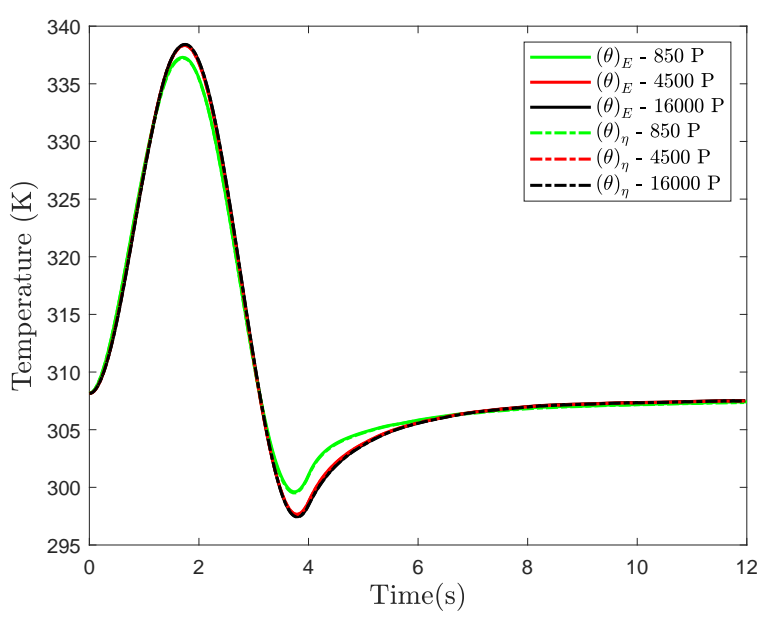

(b)

Figure 12: Rotating disk: time evolution of the components of (a) vertical velocity $v_{Z}$ and (b) temperature at material point $\boldsymbol{X}=[2,4,0]^{T} \mathrm{~m}$ for different discretisations (800, 4500 and 16000 particles). Results obtained using the proposed $\{\boldsymbol{p}, \boldsymbol{F}, \boldsymbol{H}, J, E\}$ and $\{\boldsymbol{p}, \boldsymbol{F}, \boldsymbol{H}, J, \eta\}$ Upwind-SPH methodologies considering a polyconvex Mie-Grüneisen equation of state. The material properties used are Young's modulus $E=1474 \mathrm{~Pa}$, density $\rho_{R}=10 \mathrm{~kg} / \mathrm{m}^{3}$, Poisson's ratio $\nu=0.478$, thermal conductivity $h=10 \mathrm{Wm}^{-1} \mathrm{~K}^{-1}$, specific heat capacity $C_{v}=10 \mathrm{Jkg}^{-1} \mathrm{~K}^{-1}$, thermal expansion coefficient $\alpha=2.223 \times 10^{-4} \mathrm{~K}^{-1}$, the reference temperature $\theta_{R}=308.15$ $\mathrm{K}$ and $\alpha_{\mathrm{CFL}}=0.3$. 


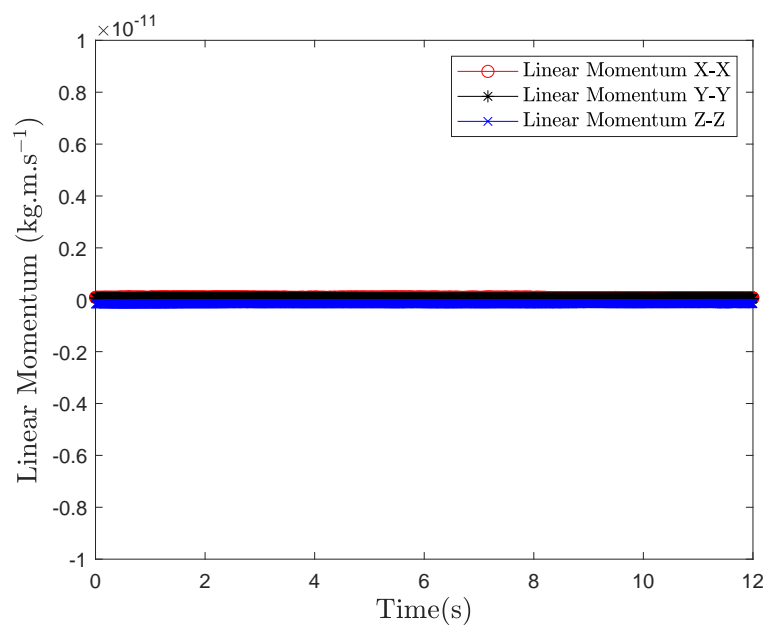

(a)

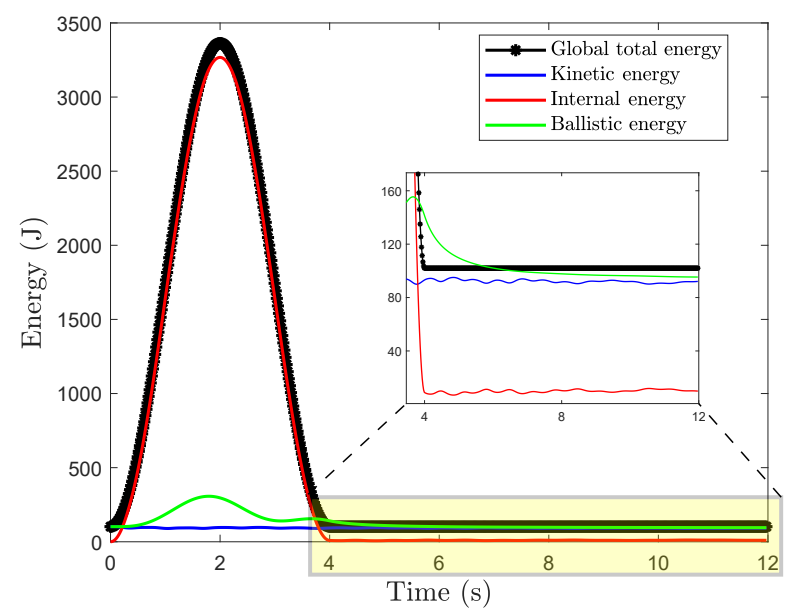

(c)

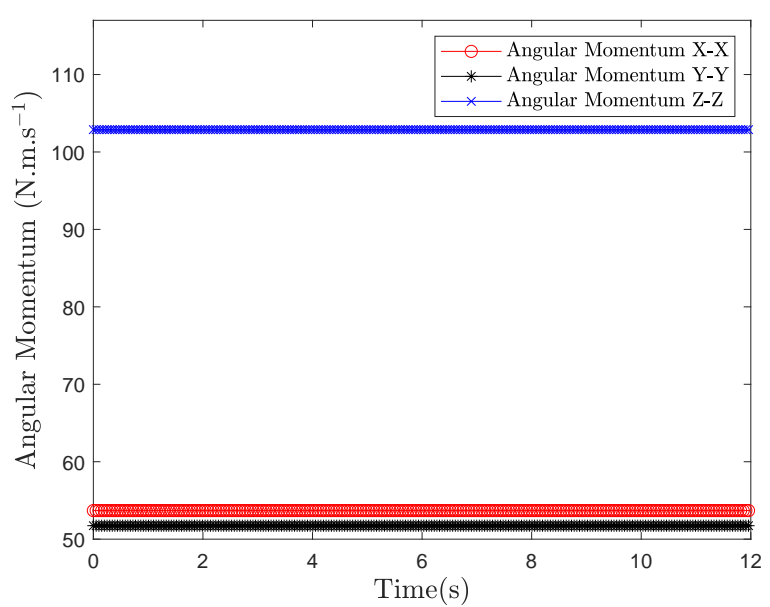

(b)

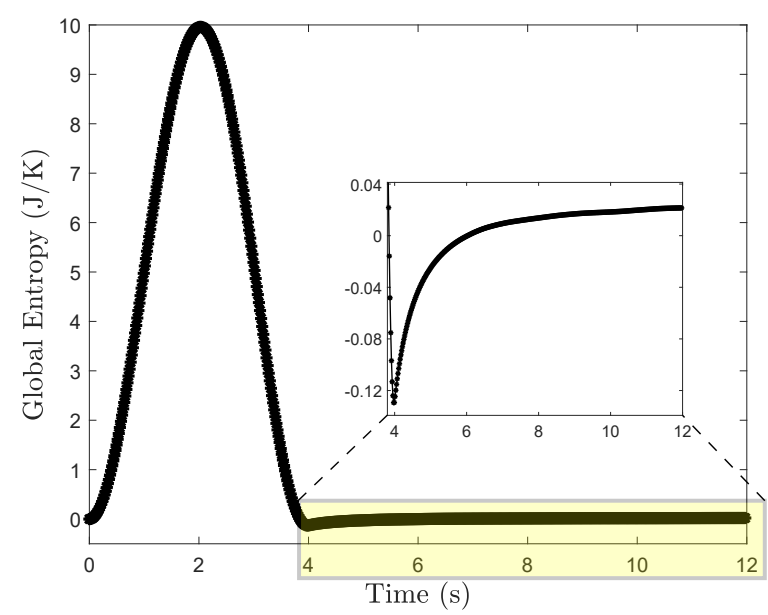

(d)

Figure 13: Rotating disk: time evolution of the components of (a) linear momentum, (b) angular momentum; (c) energy measures, and (d) global entropy. Results obtained using a polyconvex Mie-Grüneisen equation of state. The material properties used are Young's modulus $E=1474 \mathrm{~Pa}$, density $\rho_{R}=10 \mathrm{~kg} / \mathrm{m}^{3}$, Poisson's ratio $\nu=0.478$, thermal conductivity $h=10 \mathrm{Wm}^{-1} \mathrm{~K}^{-1}$, specific heat capacity $C_{v}=10 \mathrm{Jkg}^{-1} \mathrm{~K}^{-1}$, thermal expansion coefficient $\alpha=2.223 \times 10^{-4} \mathrm{~K}^{-1}$, the reference temperature $\theta_{R}=308.15 \mathrm{~K}$ and $\alpha_{\mathrm{CFL}}=0.3$. 

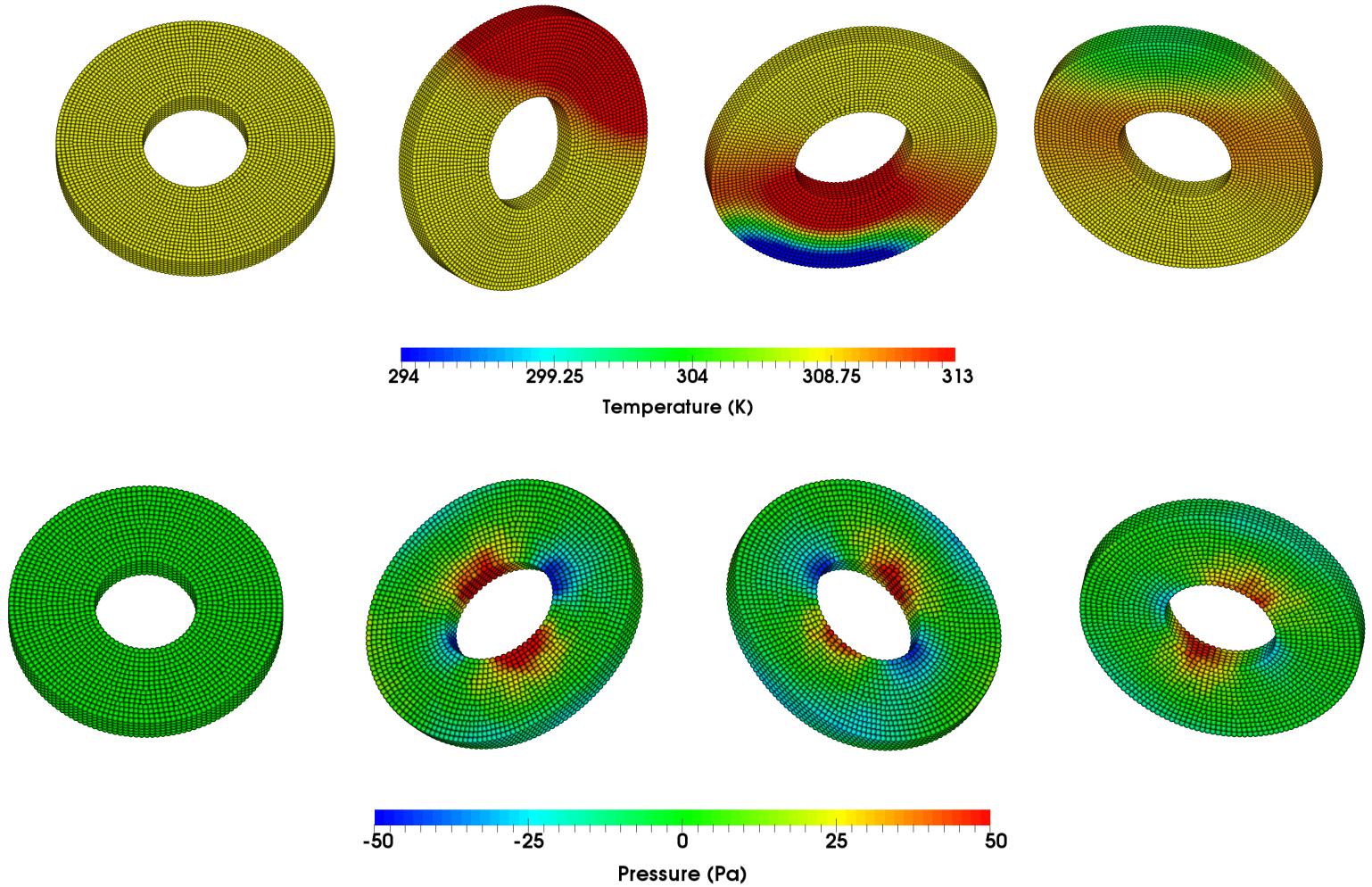

Figure 14: Rotating disk: a sequence of deformed configurations with temperature and pressure distributions at time $t=0.5 *\{0,4,9,14\} \mathrm{s}$ (left to right) and $t=3.3 *\{0,1,2,3\} \mathrm{s}$ (left to right), respectively. Results obtained using a polyconvex Mie-Grüneisen equation of state. The material properties used are Young's modulus $E=1474 \mathrm{~Pa}$, density $\rho_{R}=10 \mathrm{~kg} / \mathrm{m}^{3}$, Poisson's ratio $\nu=0.478$, thermal conductivity $h=10 \mathrm{Wm}^{-1} \mathrm{~K}^{-1}$, specific heat capacity $C_{v}=10 \mathrm{Jkg}^{-1} \mathrm{~K}^{-1}$, thermal expansion coefficient $\alpha=2.223 \times 10^{-4} \mathrm{~K}^{-1}$, the reference temperature $\theta_{R}=308.15 \mathrm{~K}$ and $\alpha_{\mathrm{CFL}}=0.3$. 


\subsection{Rotating Disk}

Similar to the objectives described in Section 7.2, another benchmark problem previously explored in References [44, 50] is considered. This example presents the (large) rotation of a free flying disk and its geometry is shown in Figure 10, In terms of initial conditions, the disk is initiated by a homogeneous temperature profile of $\left.\theta\right|_{t=0}=\theta_{R}$, and a velocity field specifically chosen as

$$
\left.\boldsymbol{v}\right|_{t=0}=\boldsymbol{\omega}_{R} \times \boldsymbol{X}, \quad \text { with } \quad \boldsymbol{\omega}_{R}=\left[\begin{array}{l}
1 \\
1 \\
1
\end{array}\right] .
$$

In addition, the disk is thermally insulated from the environment, except for a quarter of its lateral surface (see Figure 10), where a time-varying (sinusoidal) boundary heat flux $Q_{B}$ is prescribed as

$$
Q_{B}=1591.5 f(t)\left(\mathrm{W} / \mathrm{m}^{2}\right), \quad \text { where } f(t)=\left\{\begin{array}{cl}
\sin \left(\frac{\pi}{2} t\right) & t \leq 4 s \\
0 & t>4 s
\end{array}\right.
$$

A thermo-elastic Mie-Grüneisen equation of state is employed with Young's modulus $E=1474$ Pa, density $\rho_{R}=10 \mathrm{~kg} / \mathrm{m}^{3}$, Poisson's ratio $\nu=0.478$, thermal conductivity $h=10 \mathrm{Wm}^{-1} \mathrm{~K}^{-1}$, specific heat capacity $C_{v}=10 \mathrm{Jkg}^{-1} \mathrm{~K}^{-1}$, thermal expansion coefficient $\alpha=2.223 \times 10^{-4} \mathrm{~K}^{-1}$ and the reference temperature $\theta_{R}=308.15 \mathrm{~K}$.

Figure 11 illustrates a series of snapshots for different levels of discretisations (i.e. particle refinement), at time $t=1.35 \mathrm{~s}$. It is evident how the pressure resolution is enhanced as the number of particles is increased. Figure 12 presents the comparison between the proposed $\{\boldsymbol{p}, \boldsymbol{F}, \boldsymbol{H}, J, E\}$ and $\{\boldsymbol{p}, \boldsymbol{F}, \boldsymbol{H}, J, \eta\}$ Upwind-SPH methodologies. The time evolution of both the vertical component of the velocity $v_{Z}$ and the temperature are monitored at position $\boldsymbol{X}=$ $[2,4,0]^{T} \mathrm{~m}$. Very similar (almost identical) results are obtained.

As expected, Figure $13 \mathrm{a}$ shows the components of linear momentum preserve up to zero machine accuracy. The evolution of the components of angular momentum are also shown in Figure 13b, where the initial values are conserved. Figure $13 \mathrm{c}$ presents the time history of the different energetic contributions. As it can be seen, the total energy initially increases and then decreases as a result of the applied boundary heat flux (93). This is expected as the disk only exchanges heat through a quarter of its boundary (in which the heat flux is imposed) and the rest of the body is insulated. For this reason, the total energy (see Figure 13c) has the exact same profile as the prescribed (sinusoidal) heat flux and, more importantly, remains almost constant after $t=4 \mathrm{~s}$ due to the removal of heat flux. Once the system is isolated (after $t=4 \mathrm{~s}$ ), the ballistic energy decreases over time, whereby the irreversibility is caused by heat conduction and numerical dissipation, which can be understood as the satisfaction of the second law of thermodynamics. Similarly, the fluctuations of the total kinetic energy and the total internal energy are gradually damped due to the diffusive nature of the heat conduction.

For completeness, the time evolution of the deformed disk is depicted in Figure 14. The implication of (outflow) heat flux described in (93) can be clearly observed through the evolution of the temperature field. Additionally, the pressure pattern indicates the contraction and expansion of the inner circle of the disk during deformation process. 


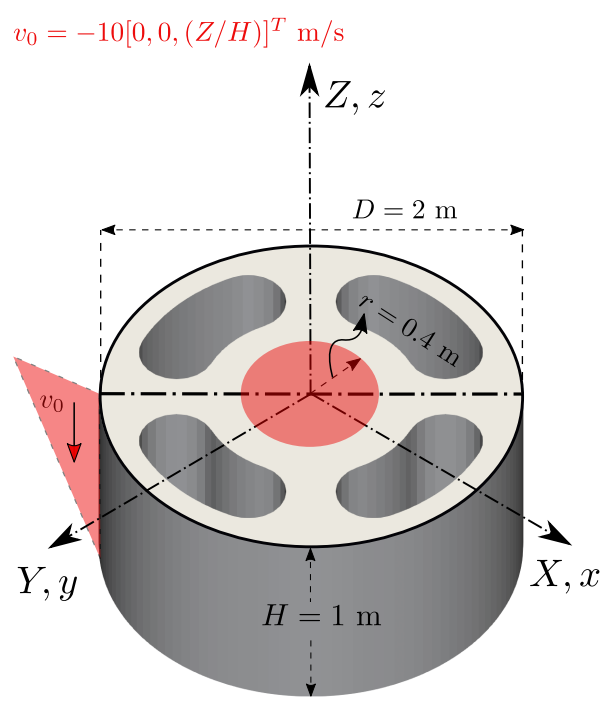

(a) Initial configuration

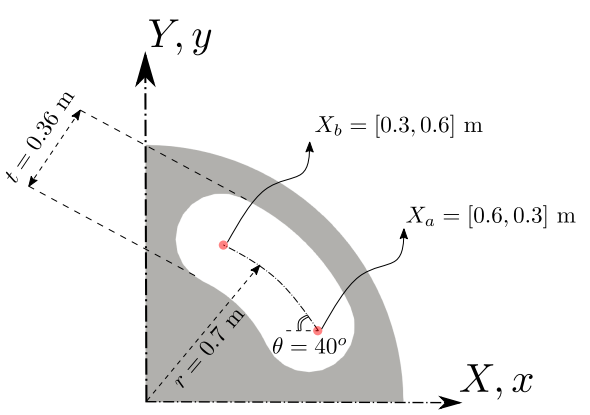

(b) Planar $1 / 4^{\text {th }}$ geometry

Figure 15: Punch test: problem setup

\subsection{Punch test}

As displayed in Figure 15, a perforated cylinder is initially punched on the middle-top face with a linear velocity field described as

$$
\left.\boldsymbol{v}\right|_{t=0}=-10\left[\begin{array}{c}
0 \\
0 \\
\frac{Z}{H}
\end{array}\right] \mathrm{m} / \mathrm{s}
$$

where $H=1 \mathrm{~m}$. Symmetric boundary conditions (i.e. roller support) are set on the bottom surface of the cylinder whilst the rest of the boundaries are left free. The cylinder, with an initial homogeneous temperature profile $\left.\theta\right|_{t=0}=\theta_{R}$, is thermally insulated from the environment. The objective of this example is to show the capability of the $\{\boldsymbol{p}, \boldsymbol{F}, \boldsymbol{H}, J, E\}$ Upwind-SPH algorithm in alleviating spurious pressure modes in the vicinity of near incompressibility. A polyconvex Mie-Grüneisen model is employed with material properties Young's modulus $E=50.05 \mathrm{KPa}$, density $\rho_{R}=1000 \mathrm{~kg} / \mathrm{m}^{3}$, Poisson's ratio $\nu=0.499$, thermal conductivity $h=10 \mathrm{Wm}^{-1} \mathrm{~K}^{-1}$, specific heat capacity $C_{v}=1 \mathrm{Jkg}^{-1} \mathrm{~K}^{-1}$, thermal expansion coefficient $\alpha=2.223 \times 10^{-4} \mathrm{~K}^{-1}$ and reference temperature $\theta_{R}=293.15 \mathrm{~K}$.

In Figure 16, it is evident that the reduced version $\{\boldsymbol{p}, \boldsymbol{F}, \boldsymbol{H}, E\}$ of the Upwind-SPH methodology suffers from spurious temperature/pressure modes. The numerical oscillations, however, are immediately removed by using the complete Upwind-SPH $\{\boldsymbol{p}, \boldsymbol{F}, \boldsymbol{H}, J, E\}$ algorithm. Specifically, the geometric conservation $J$ equation is proven to be extremely useful when attempting to alleviate these spurious pressure modes, especially in the case of a nearly incompressible material. Figure 17 depicts a comparison between the proposed SPH methodology and the in-house Upwind-VCFVM [43, where excellent agreement is observed.

For qualitative comparison purposes, the time history of the vertical velocity $v_{Z}$ and the component $P_{11}$ of the stress tensor are monitored. In this Figure 18, two proposed methodologies are compared, namely the $\{\boldsymbol{p}, \boldsymbol{F}, \boldsymbol{H}, J, E\}$ Upwind-SPH and the $\{\boldsymbol{p}, \boldsymbol{F}, \boldsymbol{H}, J, \eta\}$ Upwind-SPH. Practically identical results are seen for both algorithms. Figure 19 shows a time sequence of the deformed structure, indicating smooth pressure and temperature fields. 

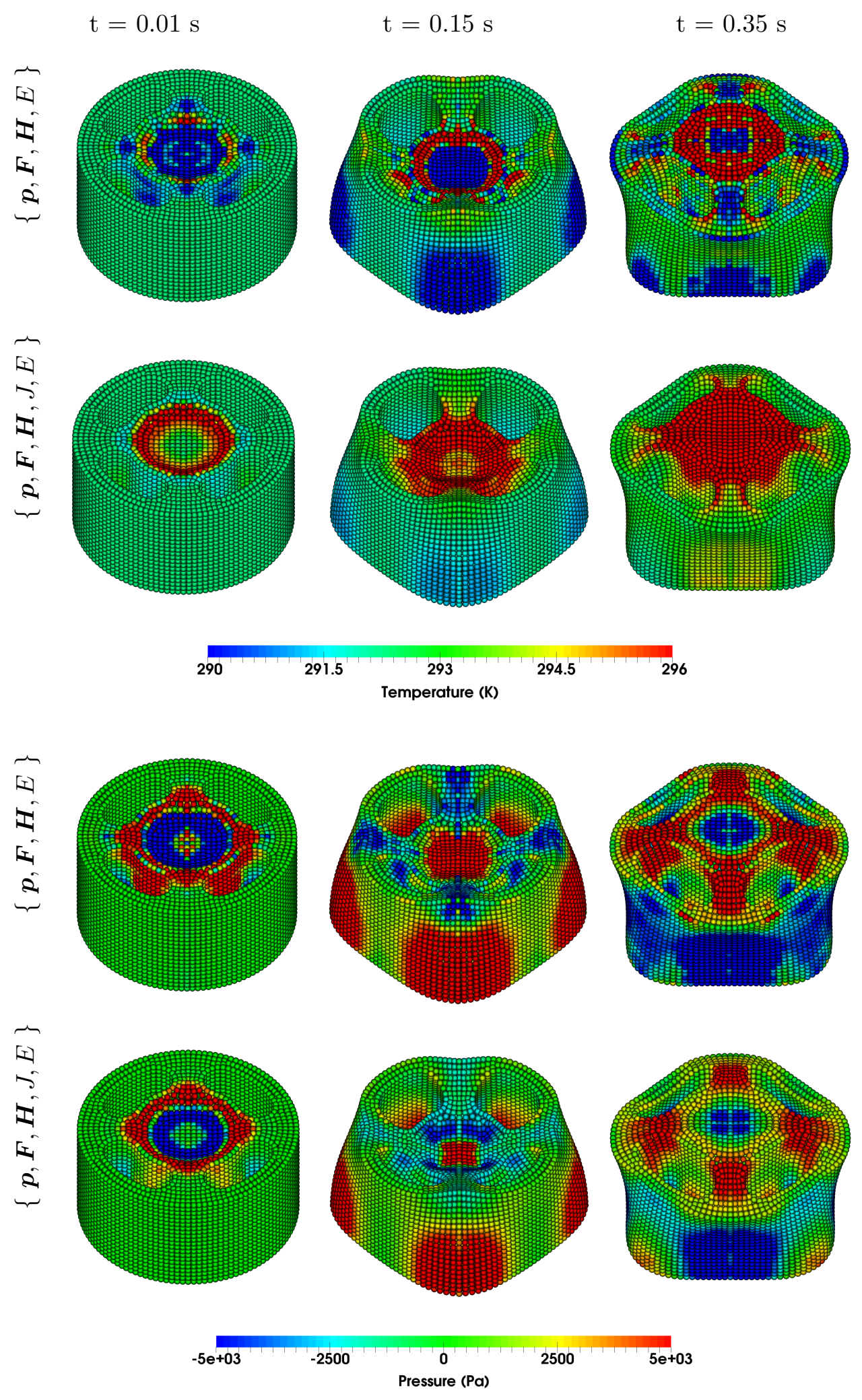

Figure 16: Punch test: a sequence of deformed configurations with temperature and pressure distributions using the reduced version $\{\boldsymbol{p}, \boldsymbol{F}, \boldsymbol{H}, E\}$ and a complete $\{\boldsymbol{p}, \boldsymbol{F}, \boldsymbol{H}, J, E\}$ Upwind-SPH methodologies. Results obtained using a polyconvex Mie-Grüneisen equation of state. The material properties used are Young's modulus $E=50.05 \mathrm{KPa}$, density $\rho_{R}=1000 \mathrm{~kg} / \mathrm{m}^{3}$, Poisson's ratio $\nu=0.499$, thermal conductivity $h=10$ $\mathrm{Wm}^{-1} \mathrm{~K}^{-1}$, specific heat capacity $C_{v}=1 \mathrm{Jkg}^{-1} \mathrm{~K}^{-1}$, thermal expansion coefficient $\alpha=2.223 \times 10^{-4} \mathrm{~K}^{-1}$, reference temperature $\theta_{R}=293.15 \mathrm{~K}$ and $\alpha_{\mathrm{CFL}}=0.3$. 

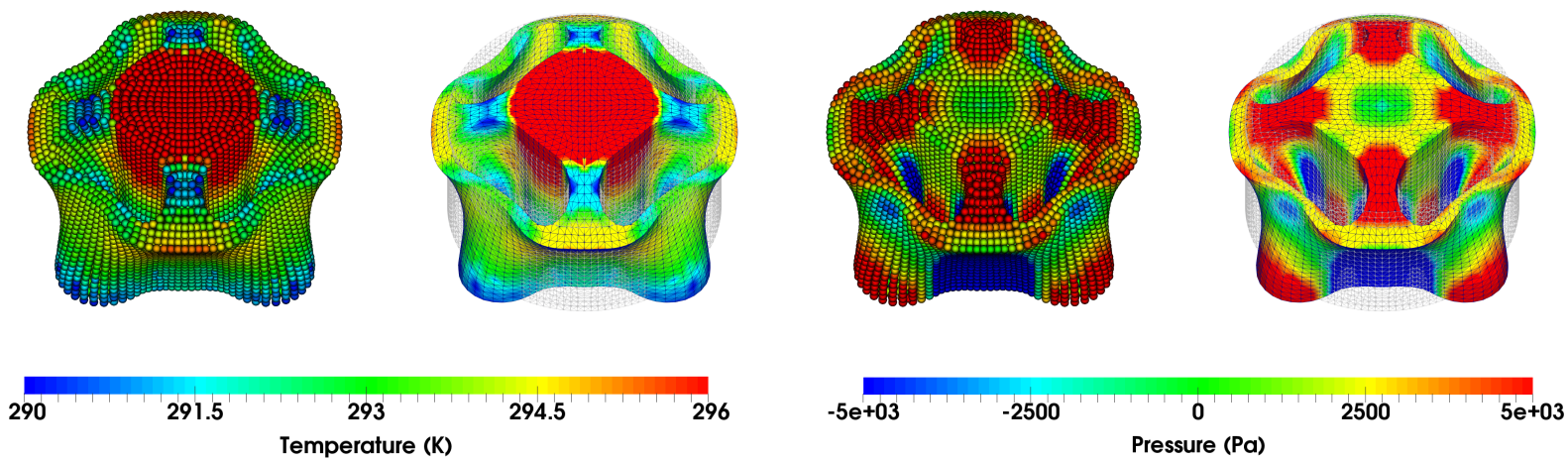

Figure 17: Punch test: comparison of deformed shapes plotted with pressure and temperature distributions using the $\{\boldsymbol{p}, \boldsymbol{F}, \boldsymbol{H}, J, E\}$ Upwind-SPH and $\{\boldsymbol{p}, \boldsymbol{F}, \boldsymbol{H}, J, E\}$ Upwind-VCFVM at time $t=0.38 \mathrm{~s}$. Results are obtained using a polyconvex Mie-Grüneisen equation of state. The material properties used are Young's modulus $E=50.05 \mathrm{KPa}$, density $\rho_{R}=1000 \mathrm{~kg} / \mathrm{m}^{3}$, Poisson's ratio $\nu=0.499$, thermal conductivity $h=10$ $\mathrm{Wm}^{-1} \mathrm{~K}^{-1}$, specific heat capacity $C_{v}=1 \mathrm{Jkg}^{-1} \mathrm{~K}^{-1}$, thermal expansion coefficient $\alpha=2.223 \times 10^{-4} \mathrm{~K}^{-1}$, reference temperature $\theta_{R}=293.15 \mathrm{~K}$ and $\alpha_{\mathrm{CFL}}=0.3$.

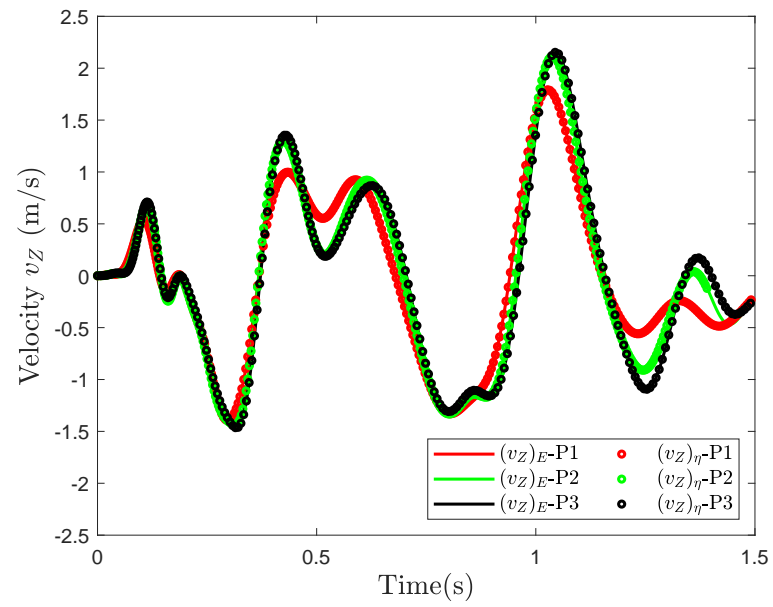

(a)

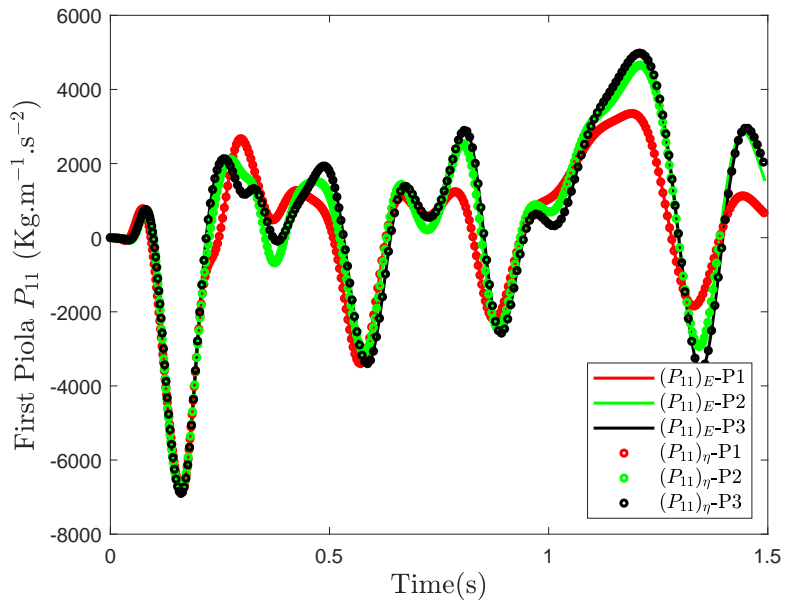

(b)

Figure 18: Punch test: time evolution of (a) component of velocity $v_{Z}$ and (b) component of first PiolaKirchhoff stress tensor $P_{11}$ at material point $\boldsymbol{X}=[0,1,1]^{T} \mathrm{~m}$. A comparison is carried out between the \{ $\boldsymbol{p}, \boldsymbol{F}, \boldsymbol{H}, J, E\}$ Upwind-SPH and $\{\boldsymbol{p}, \boldsymbol{F}, \boldsymbol{H}, J, \eta\}$ Upwind-SPH methodologies. A polyconvex Mie-Grüneisen equation of state with properties Young's modulus $E=50.05 \mathrm{KPa}$, density $\rho_{R}=1000 \mathrm{~kg} / \mathrm{m}^{3}$, Poisson's ratio $\nu=0.499$, thermal conductivity $h=10 \mathrm{Wm}^{-1} \mathrm{~K}^{-1}$, specific heat capacity $C_{v}=1 \mathrm{Jkg}^{-1} \mathrm{~K}^{-1}$, thermal expansion coefficient $\alpha=2.223 \times 10^{-4} \mathrm{~K}^{-1}$, reference temperature $\theta_{R}=293.15 \mathrm{~K}$ and $\alpha_{\mathrm{CFL}}=0.3$. $\{\mathrm{P} 1, \mathrm{P} 2, \mathrm{P} 3\}$ comprise $\{2500,5000,10000\}$ number of particles, respectively. 

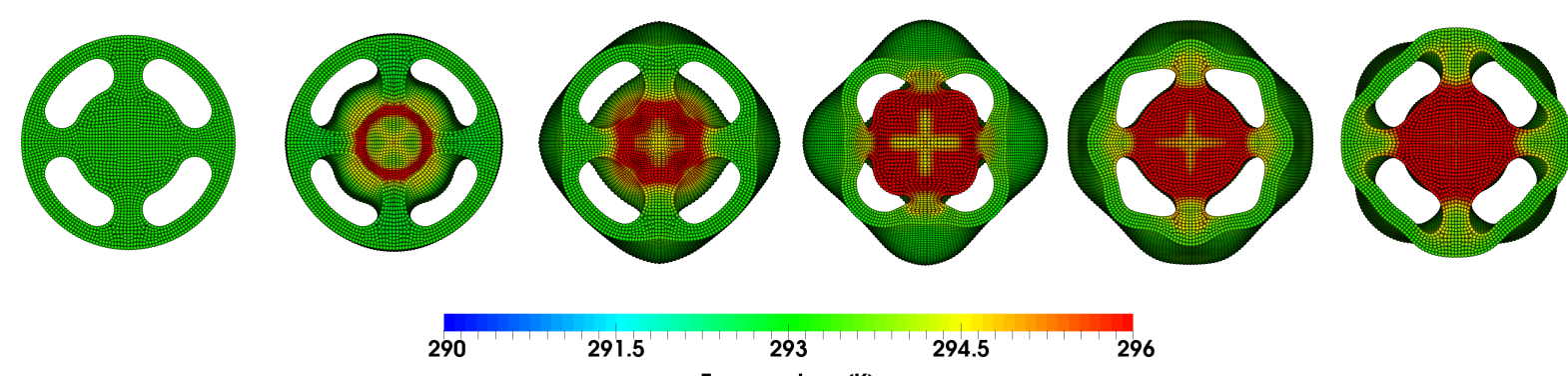

291.5

293

\begin{abstract}
Temperature (K)
\end{abstract}
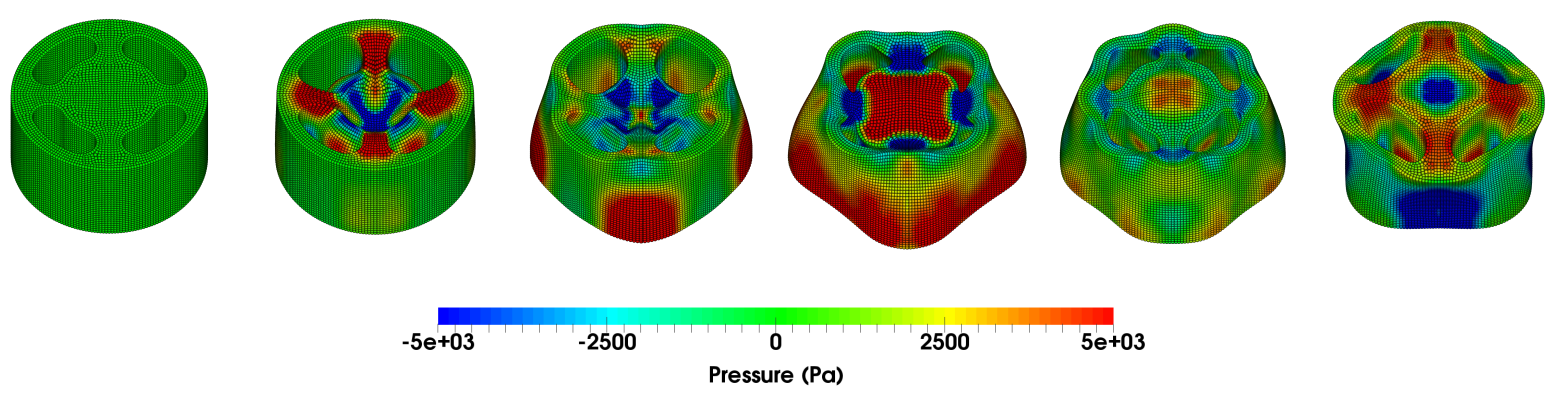

Figure 19: Punch test: a sequence of deformed configurations with temperature (top view) and pressure (isometric view) distributions at time $t=0.07 *\{0,1,2,3,4,5\} \mathrm{s}$ (left to right), respectively. Results obtained using a polyconvex Mie-Grüneisen equation of state. The material properties used are Young's modulus $E=50.05$ $\mathrm{KPa}$, density $\rho_{R}=1000 \mathrm{~kg} / \mathrm{m}^{3}$, Poisson's ratio $\nu=0.499$, thermal conductivity $h=10 \mathrm{Wm}^{-1} \mathrm{~K}^{-1}$, specific heat capacity $C_{v}=1 \mathrm{Jkg}^{-1} \mathrm{~K}^{-1}$, thermal expansion coefficient $\alpha=2.223 \times 10^{-4} \mathrm{~K}^{-1}$, reference temperature $\theta_{R}=293.15 \mathrm{~K}$ and $\alpha_{\mathrm{CFL}}=0.3$. Discretisation of 5000 number of particles.

\title{
7.5. Gripper
}

In this example, we study the thermo-elastic deformation of a gripper-like structure (see Figure 20) widely used in soft robotic applications. The main objective of this example is to show the robustness of the proposed $\{\boldsymbol{p}, \boldsymbol{F}, \boldsymbol{H}, J, E\}$ Upwind-SPH algorithm in a bending dominated scenario. The gripper has four geometrically symmetric arms with $L=0.4 \mathrm{~m}$ which are connected to a hollow cylindrical shape with radius of $R=0.1 \mathrm{~m}$. The central interior of the cylinder has a constant temperature of $\theta=280 \mathrm{~K}$ (thermal Dirichlet boundary condition) while the rest of the domain boundaries are regarded as thermally insulated from the environment. Additionally, the four end faces of the arms are also subjected to a constant vertical traction $t_{B}=1000 \mathrm{~N} / \mathrm{m}^{2}$ where the temperature is initially set to be $\theta=300 \mathrm{~K}$. For the rest of the domain, an initial temperature distribution of $\theta_{R}=293.15 \mathrm{~K}$ is prescribed.

A polyconvex Mie-Grüneisen equation of state is employed. The material properties used are Young's modulus $E=50.05 \mathrm{KPa}$, density $\rho_{R}=1000 \mathrm{~kg} / \mathrm{m}^{3}$, Poisson's ratio $\nu=0.45$, thermal conductivity $h=10 \mathrm{Wm}^{-1} \mathrm{~K}^{-1}$, specific heat capacity $C_{v}=1 \mathrm{Jkg}^{-1} \mathrm{~K}^{-1}$ and thermal expansion coefficient $\alpha=2.223 \times 10^{-4} \mathrm{~K}^{-1}$.

Figure 21 shows, for a given time step, the deformation of the gripper from three different viewpoints, namely isometric view, front view and top/bottom view. Very smooth pressure and temperature distributions are also observed. Notice that heat conduction occurs through the four arms of the gripper as a result of the temperature difference within the structure. For completeness, the time history of the components of displacement at position $\boldsymbol{X}=[0.4,0,-0.03]^{T}$ $\mathrm{m}$ (i.e. one end of the gripper) are also monitored in Figure 22. 


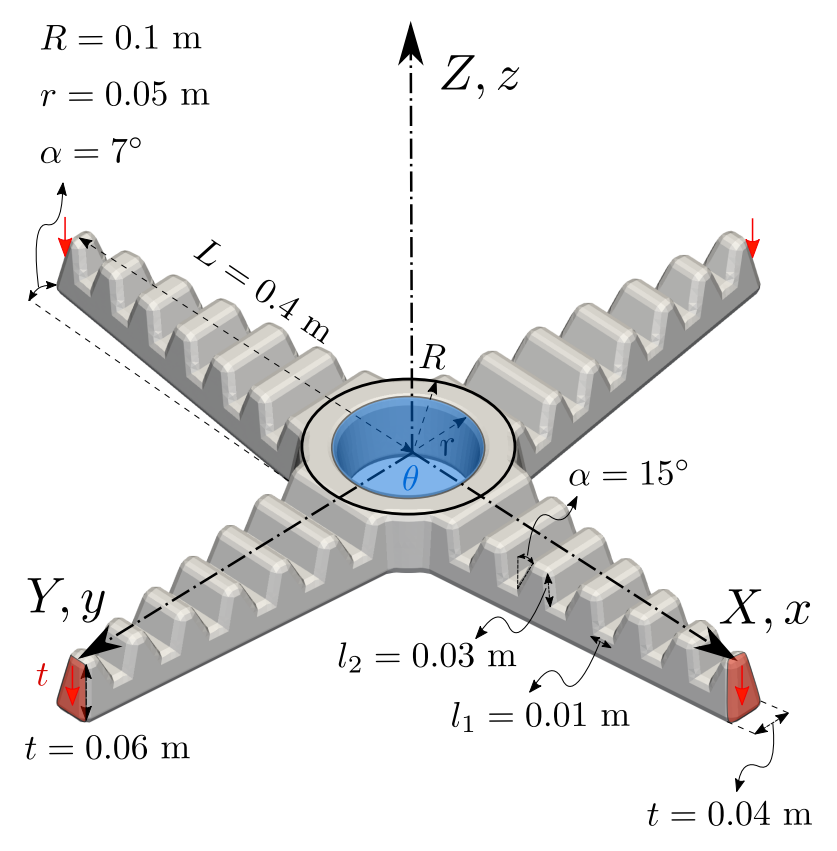

Figure 20: Gripper: problem setup

\subsection{Spherical ball}

In this last example, we demonstrate the robustness of the $\{\boldsymbol{p}, \boldsymbol{F}, \boldsymbol{H}, J, E\}$ Upwind-SPH algorithm on a thin-walled structure. The geometry and problem setup are shown in Figure 23. A spherical ball, with an unsymmetrical hole pattern, is initially punched on its top surface with a velocity field of

$$
\left.\boldsymbol{v}\right|_{t=0}=\left[\begin{array}{c}
0 \\
0 \\
-1.5
\end{array}\right] \mathrm{m} / \mathrm{s}
$$

For consideration of thermal effects, the ball is initially subjected to a uniform temperature profile of $\left.\theta\right|_{t=0}=\theta_{R}$, in combination with a boundary heat flux prescribed on the inner surface of the ball as

$$
Q_{B}(t)=470.871\left(\mathrm{KW} / \mathrm{m}^{2}\right) \quad t \geq 0 \mathrm{~s},
$$

A polyconvex modified entropic elasticity is used with the material properties chosen as Young's modulus $E=17 \mathrm{MPa}$, density $\rho_{R}=1100 \mathrm{~kg} / \mathrm{m}^{3}$, Poisson's ratio $\nu=0.3$, thermal conductivity $h=0.2 \mathrm{Wm}^{-1} \mathrm{~K}^{-1}$, specific heat capacity $C_{v}=1000 \mathrm{Jkg}^{-1} \mathrm{~K}^{-1}$, thermal expansion coefficient $\alpha=2.223 \times 10^{-4} \mathrm{~K}^{-1}$ and reference temperature $\theta_{R}=293.15 \mathrm{~K}$.

Figure 24 shows the time evolution of the deformations of a ball. Again, very smooth pressure and temperature profiles are seen throughout the entire structure. Neither hourglassing nor spurious instabilities are observed.

\section{Conclusions}

In this paper, a new Smooth Particle Hydrodynamics computational framework bas been introduced for the analysis of fast transient solid dynamics in the context of thermo-elasticity. In addition to conservation laws for the linear momentum $\boldsymbol{p}$, the deformation gradient $\boldsymbol{F}$, its cofactor $\boldsymbol{H}$ and its Jacobian $J$, previously used [1] in isothermal elasticity, a further conservation 

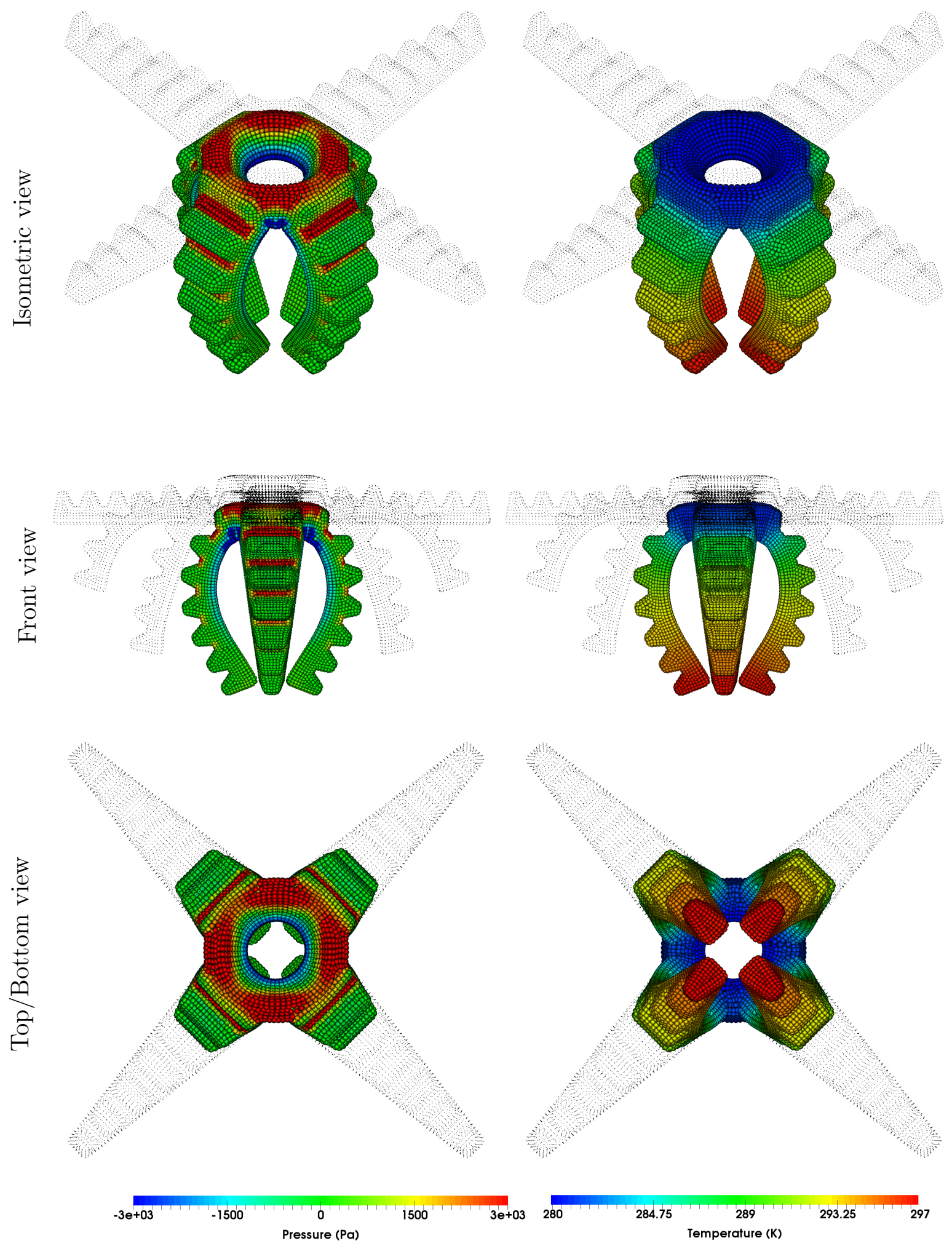

Figure 21: Gripper: a sequence of deformed configurations with temperature and pressure distributions at $t=0.47 \mathrm{~s}$. Results obtained using a polyconvex Mie-Grüneisen equation of state. The material properties used are Young's modulus $E=50.05 \mathrm{KPa}$, density $\rho_{R}=1000 \mathrm{~kg} / \mathrm{m}^{3}$, Poisson's ratio $\nu=0.45$, thermal conductivity $h=10 \mathrm{Wm}^{-1} \mathrm{~K}^{-1}$, specific heat capacity $C_{v}=1 \mathrm{Jkg}^{-1} \mathrm{~K}^{-1}$, thermal expansion coefficient $\alpha=2.223 \times 10^{-4} \mathrm{~K}^{-1}$ and $\alpha_{\mathrm{CFL}}=0.3$. 


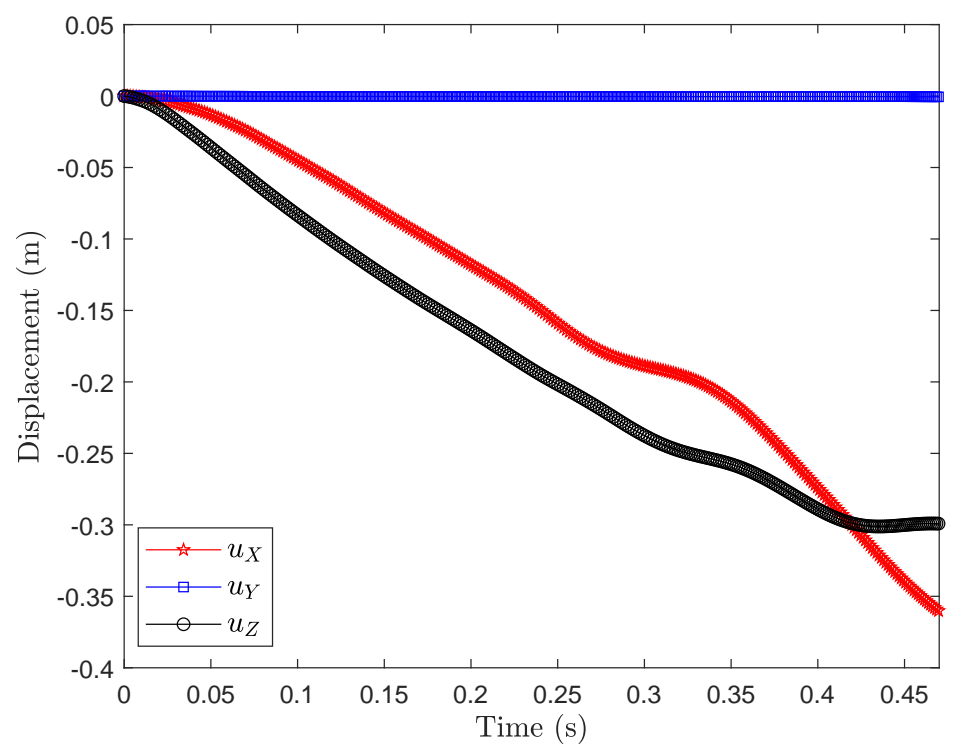

Figure 22: Gripper: time evolution of the components of displacement profile at material point $\boldsymbol{X}=$ $[0.4,0,-0.03]^{T} \mathrm{~m}$. Results obtained using a polyconvex Mie-Grüneisen equation of state. The material properties used are Young's modulus $E=50.05 \mathrm{KPa}$, density $\rho_{R}=1000 \mathrm{~kg} / \mathrm{m}^{3}$, Poisson's ratio $\nu=0.45$, thermal conductivity $h=10 \mathrm{Wm}^{-1} \mathrm{~K}^{-1}$, specific heat capacity $C_{v}=1 \mathrm{Jkg}^{-1} \mathrm{~K}^{-1}$, thermal expansion coefficient $\alpha=2.223 \times 10^{-4} \mathrm{~K}^{-1}$ and $\alpha_{\mathrm{CFL}}=0.3$.

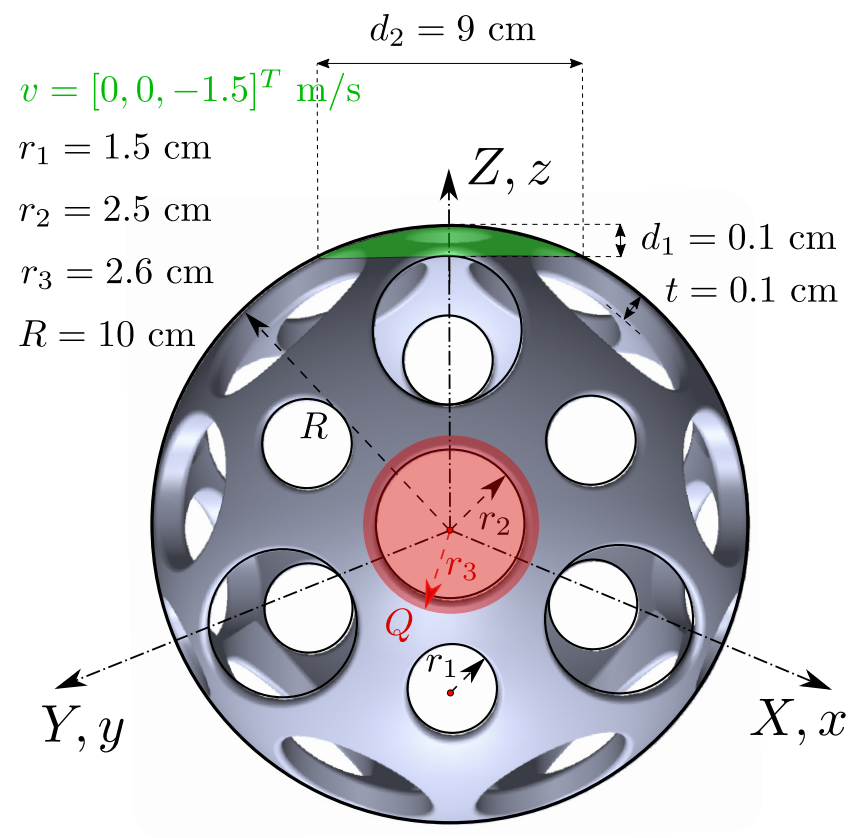

Figure 23: Spherical ball: problem setup 

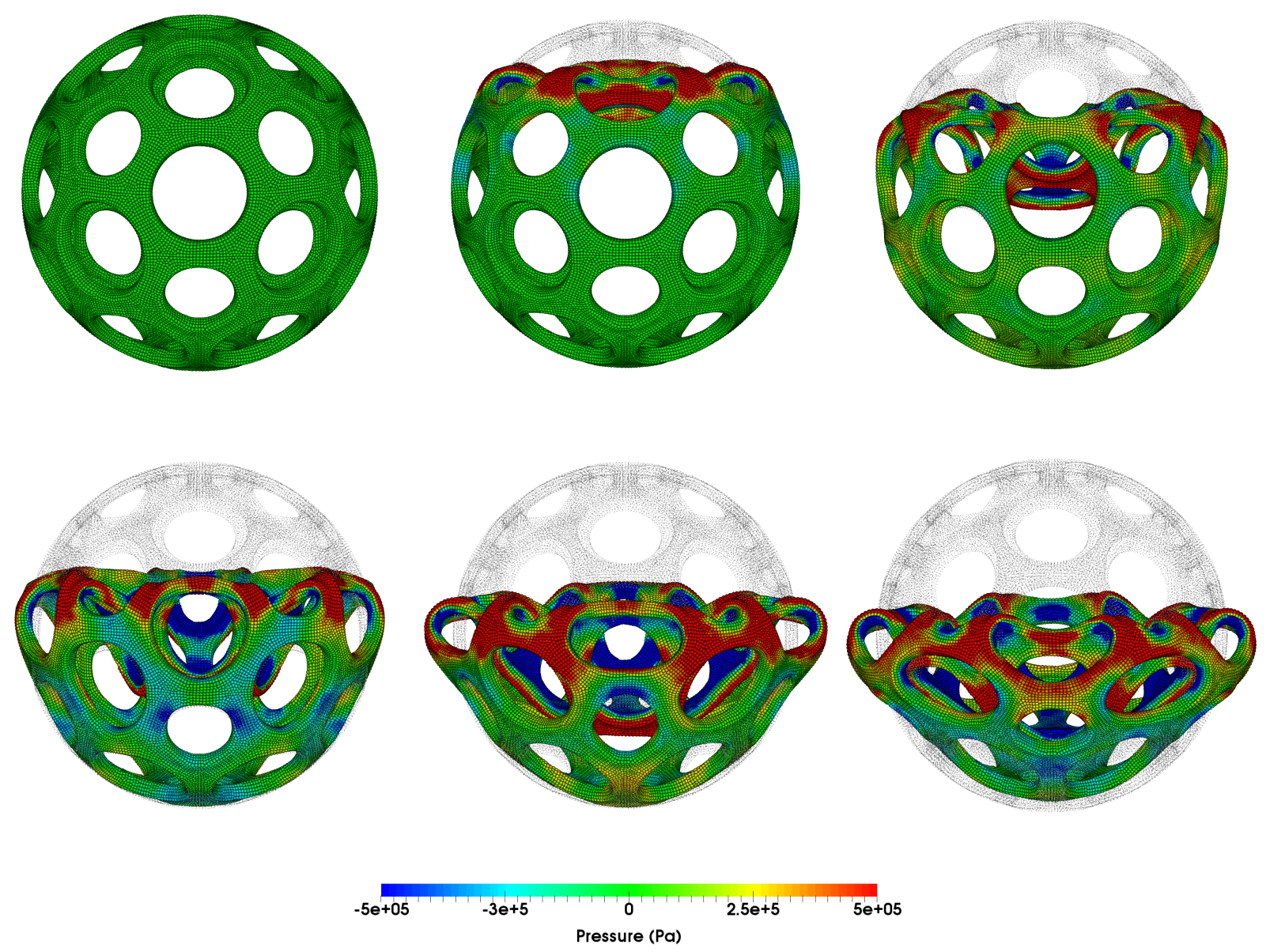

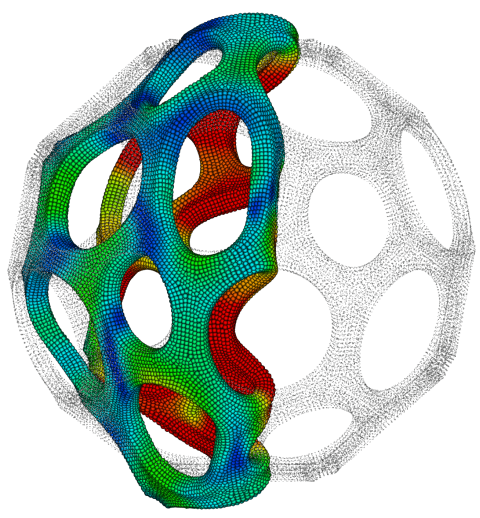

Side view

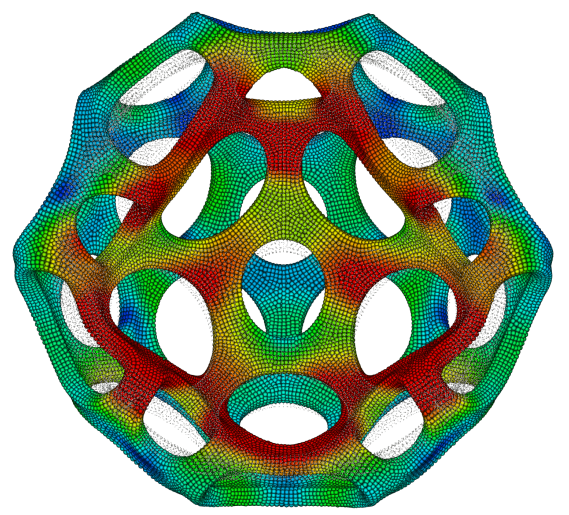

Top view

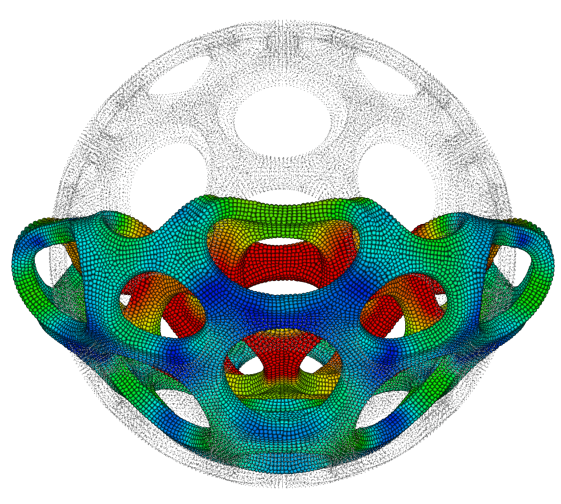

Front view

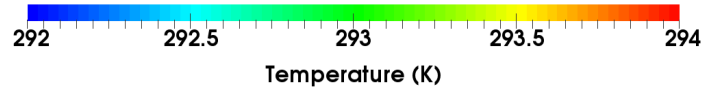

Figure 24: Spherical ball: a sequence of deformed configurations with temperature and pressure distributions at time $t=0.042 \mathrm{~s}$ and $t=0.01 *\{0,1,2,3,4,4.2\} \mathrm{s}$ (left to right-top to bottom), respectively. Results obtained using a polyconvex modified entropic-elastic model. The material properties used are Young's modulus $E=17$ $\mathrm{MPa}$, density $\rho_{R}=1100 \mathrm{~kg} / \mathrm{m}^{3}$, Poisson's ratio $\nu=0.3$, thermal conductivity $h=0.2 \mathrm{Wm}^{-1} \mathrm{~K}^{-1}$, specific heat capacity $C_{v}=1000 \mathrm{Jkg}^{-1} \mathrm{~K}^{-1}$, thermal expansion coefficient $\alpha=2.223 \times 10^{-4} \mathrm{~K}^{-1}$ and $\alpha_{\mathrm{CFL}}=0.3$. 
law, representing the first law of thermodynamics in terms of the total energy density $E$, is incorporated to extend the range of applications into thermally coupled hyperelasticity.

From the continuum standpoint, the methodology is built upon the careful definition of polyconvex internal energy density functionals with respect to the extended set $\{\boldsymbol{F}, \boldsymbol{H}, J, \eta\}$ by establishing sufficient conditions on the internal energy density and the entropy, when both are measured at a reference temperature. From the discretisation point of view, an explicit Smooth Particle Hydrodynamics is presented where consistent Riemann-based (upwinding) numerical stabilisation is introduced satisfying local numerical entropy production, the latter demonstrated by monitoring the so-called ballistic energy of the system.

A wide spectrum of numerical examples is presented in order to assess the applicability and robustness of the proposed formulation. Crucially, both velocities, stresses (volumetric and deviatoric) and total energy display the same (second order) rate of convergence. For the sake of completeness, the scheme is compared against an alternative and equally competitive entropy-based implementation $\{\boldsymbol{p}, \boldsymbol{F}, \boldsymbol{H}, J, \eta\}$.

\section{Appendix A. Derivation of suitable conjugate entropy variables to $\{p, F, H, J, E\}$}

In the context of thermo-elasticity, a generalised convex entropy function, namely the ballistic energy, is expressed in terms of linear momentum $\boldsymbol{p}$, the triplet of deformation measures $\mathcal{X}=\{\boldsymbol{F}, \boldsymbol{H}, J\}$ and total energy density $E$ as ${ }^{15}$

$$
B\left(\boldsymbol{p}, \mathcal{X}_{E}\right)=E-\theta_{R} \hat{\eta}\left(\boldsymbol{p}, \mathcal{X}_{E}\right) ; \quad \mathcal{X}_{E}=\{\mathcal{X}, E\}
$$

Recalling that $\hat{\mathcal{E}}\left(\boldsymbol{\mathcal { X }}, \hat{\eta}\left(\boldsymbol{p}, \boldsymbol{\mathcal { X }}_{E}\right)\right)=E-\frac{1}{2 \rho_{R}} \boldsymbol{p} \cdot \boldsymbol{p}$ [50], the entropy $\hat{\eta}\left(\boldsymbol{p}, \boldsymbol{\mathcal { X }}_{E}\right)$ is obtained implicitly via the expression $\varphi\left(\boldsymbol{p}, \mathcal{X}_{E}, \hat{\eta}\right)$ generally defined as [76]

$$
0=\varphi\left(\boldsymbol{p}, \boldsymbol{\mathcal { X }}_{E}, \hat{\eta}\right)=\hat{\mathcal{E}}(\boldsymbol{\mathcal { X }}, \hat{\eta})-\left(E-\frac{1}{2 \rho_{R}} \boldsymbol{p} \cdot \boldsymbol{p}\right)
$$

Differentiating $B\left(\boldsymbol{p}, \boldsymbol{\mathcal { X }}_{E}\right)$ A.1 gives the conjugate entropy variables $\mathcal{V}$ as

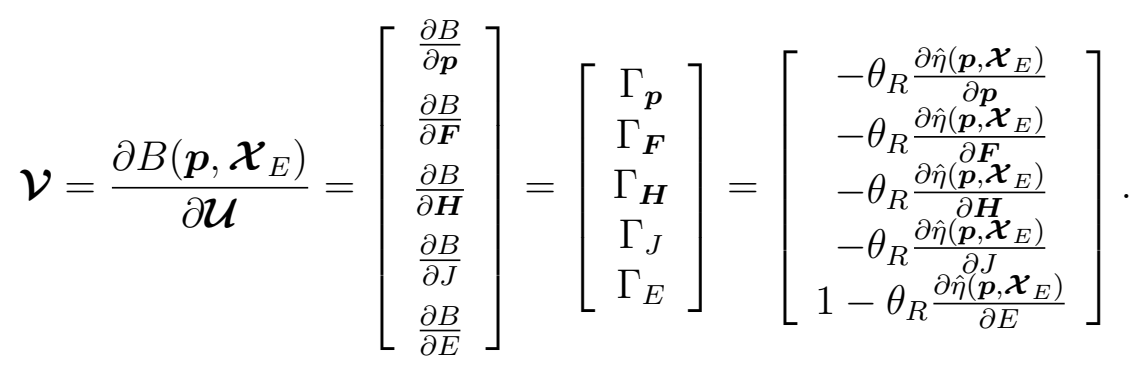

Moreover, derivatives of $\hat{\eta}\left(\boldsymbol{p}, \boldsymbol{\mathcal { X }}_{E}\right)$ can now be obtained by differentiating $\varphi$ A.2 with respect to each component of $\mathcal{U}$. For instance, the derivative of $\hat{\eta}\left(\boldsymbol{p}, \boldsymbol{\mathcal { X }}_{E}\right)$ with respect to $\boldsymbol{p}$ is achieved using the chain rule to give

$$
\mathbf{0}=\frac{\partial \varphi\left(\boldsymbol{p}, \boldsymbol{\mathcal { X }}_{E}, \hat{\eta}\left(\boldsymbol{p}, \boldsymbol{\mathcal { X }}_{E}\right)\right)}{\partial \boldsymbol{p}}+\frac{\partial \varphi\left(\boldsymbol{p}, \boldsymbol{\mathcal { X }}_{E}, \hat{\eta}\right)}{\partial \hat{\eta}} \frac{\partial \hat{\eta}\left(\boldsymbol{p}, \boldsymbol{\mathcal { X }}_{E}\right)}{\partial \boldsymbol{p}}
$$

\footnotetext{
${ }^{15}$ As shown by Wagner in [76, the function $-\hat{\eta}\left(\boldsymbol{p}, \mathcal{X}_{E}\right)$ is convex in $\boldsymbol{p}$ and $\boldsymbol{\mathcal { X }}_{E}$.
} 
Since $\frac{\partial \varphi\left(\boldsymbol{p}, \boldsymbol{\mathcal { X }}_{E}, \hat{\eta}\right)}{\partial \hat{\eta}}=\frac{\partial \hat{\mathcal{E}}(\boldsymbol{\mathcal { X }}, \hat{\eta})}{\partial \hat{\eta}}=\theta$, equation above after rearranging becomes

$$
-\frac{\partial \hat{\eta}\left(\boldsymbol{p}, \boldsymbol{\mathcal { X }}_{E}\right)}{\partial \boldsymbol{p}}=\left(\frac{\partial \varphi}{\partial \hat{\eta}}\right)^{-1} \frac{\partial \varphi}{\partial \boldsymbol{p}}=\frac{\boldsymbol{v}}{\theta}
$$

Repeating the same procedure on the derivatives of $\hat{\eta}$ with respect to $\boldsymbol{F}, \boldsymbol{H}, J$ and $E$, results in

$$
\begin{aligned}
-\frac{\partial \hat{\eta}\left(\boldsymbol{p}, \boldsymbol{\mathcal { X }}_{E}\right)}{\partial \boldsymbol{F}} & =\left(\frac{\partial \varphi}{\partial \hat{\eta}}\right)^{-1} \frac{\partial \varphi}{\partial \boldsymbol{F}}=\frac{1}{\theta} \boldsymbol{\Sigma}_{\boldsymbol{F}} ; \\
-\frac{\partial \hat{\eta}\left(\boldsymbol{p}, \boldsymbol{\mathcal { X }}_{E}\right)}{\partial \boldsymbol{H}} & =\left(\frac{\partial \varphi}{\partial \hat{\eta}}\right)^{-1} \frac{\partial \varphi}{\partial \boldsymbol{H}}=\frac{1}{\theta} \boldsymbol{\Sigma}_{\boldsymbol{H}} ; \\
-\frac{\partial \hat{\eta}\left(\boldsymbol{p}, \boldsymbol{\mathcal { X }}_{E}\right)}{\partial J} & =\left(\frac{\partial \varphi}{\partial \hat{\eta}}\right)^{-1} \frac{\partial \varphi}{\partial J}=\frac{1}{\theta} \boldsymbol{\Sigma}_{J} ; \\
-\frac{\partial \hat{\eta}\left(\boldsymbol{p}, \boldsymbol{\mathcal { X }}_{E}\right)}{\partial E} & =\left(\frac{\partial \varphi}{\partial \hat{\eta}}\right)^{-1} \frac{\partial \varphi}{\partial E}=\frac{1}{\theta} .
\end{aligned}
$$

Finally, by combining equations (A.6) and A.3), components of the conjugate entropy variables become

$$
\Gamma_{\boldsymbol{p}}=\frac{\theta_{R}}{\theta} \boldsymbol{v} ; \quad \Gamma_{\boldsymbol{F}}=\frac{\theta_{R}}{\theta} \boldsymbol{\Sigma}_{\boldsymbol{F}} ; \quad \Gamma_{\boldsymbol{H}}=\frac{\theta_{R}}{\theta} \boldsymbol{\Sigma}_{\boldsymbol{H}} ; \quad \Gamma_{J}=\frac{\theta_{R}}{\theta} \boldsymbol{\Sigma}_{J} ; \quad \Gamma_{E}=\frac{\vartheta}{\theta} .
$$

\section{Acknowledgements}

The authors would like to acknowledge the financial support received through the European Commission EACEA Agency, Framework Partnership Agreement 2013-0043 Erasmus Mundus Action 1b, as a part of the EM Joint Doctorate "Simulation in Engineering and Entrepreneurship Development (SEED)". The second author gratefully acknowledges the support provided by the EPSRC Strategic Support Package: Engineering of Active Materials by Multiscale/Multiphysics Computational Mechanics - EP/R008531/1. The third author acknowledges the financial support received through the European Training Network Protechtion (Project ID: 764636).

\section{References}

[1] C. H. Lee, A. J. Gil, G. Greto, S. Kulasegaram, J. Bonet, A new Jameson-Schmidt-Turkel Smooth Particle Hdrodynamics algorithm for large strain explicit fast dynamics, Computer Methods in Applied Mechanics and Engineering 311 (2016) 71-111.

[2] C. H. Lee, A. J. Gil, O. I. Hassan, J. Bonet, S. Kulasegaram, A variationally consistent Streamline Upwind Petrov Galerkin Smooth Particle Hydrodynamics algorithm for large strain solid dynamics, Computer Methods in Applied Mechanics and Engineering 318 (2017) 514-536.

[3] C. H. Lee, A. J. Gil, A. Ghavamian, J. Bonet, A total lagrangian upwind smooth particle hydrodynamics algorithm for large strain explicit solid dynamics, Computer Methods in Applied Mechanics and Engineering 344 (2019) 209 - 250. 
[4] L. B. Lucy, A numerical approach to the testing of the fission hypothesis, Astronomical Journal 82 (1977) 1013-1024.

[5] J. J. Monaghan, Smoothed particle hydrodynamics, Annual Review of Astronomy and Astrophysics 30 (1992) 543-574.

[6] J. Bonet, S. Kulasegaram, Remarks on tension instability of Eulerian and Lagrangian Corrected Smooth Particle Hydrodynamics (CSPH) methods, International Journal for Numerical Methods in Engineering 52 (2001) 1203-1220.

[7] J. Bonet, T.-S. Lok, Variational and momentum preservation aspects of smooth particle hydrodynamic formulations, Computer Methods in Applied Mechanics and Engineering 180 (1999) 97-115.

[8] G. C. Ganzenmüller, An hourglass control algorithm for Lagrangian Smooth Particle Hydrodynamics, Computer Methods in Applied Mechanics and Engineering 286 (2015) $87-106$.

[9] G. C. Ganzenmüller, S. Hiermaier, M. May, On the similarity of meshless discretizations of Peridynamics and Smooth-Particle Hydrodynamics, Computers and Structures 150 (2015) $71-78$.

[10] Y. Vidal, J. Bonet, A. Huerta, Stabilized updated Lagrangian corrected SPH for explicit dynamic problems, International Journal for Numerical Methods in Engineering 69 (2006) $2687-2710$.

[11] C. T. Dyka, R. P. Ingel, An approach for tension instability in Smoothed Particle Hydrodynamics (SPH), Computers and Structures 57 (1995) 573-580.

[12] M. A. Puso, J. S. Chen, E. Zywicz, W. Elmer, Meshfree and finite element nodal integration methods, International Journal for Numerical Methods in Engineering 74 (2008) 416-446.

[13] W. Elmer, J. S. Chen, M. Puso, E. Taciroglu, A stable, meshfree, nodal integration method for nearly incompressible solids, Finite Elements in Analysis and Design 51 (2012) 81-85.

[14] G. R. Johnson, R. A. Stryk, S. R. Beissel, SPH for velocity impact computations, Computer Methods in Applied Mechanics and Engineering 139 (1996) 347-373.

[15] G. R. Johnson, S. R. Beissel, Normalized smoothing functions for SPH impact computations, International Journal for Numerical Methods in Engineering 39 (1996) 2725-2741.

[16] P. W. Randles, L. D. Libersky, Smoothed Particle Hydrodynamics: Some recent improvements and applications, Computer Methods in Applied Mechanics and Engineering 139 (1996) 375-408.

[17] P. W. Randles, L. D. Libersky, Normalized SPH with stress points, International Journal for Numerical Methods in Engineering 48 (2000) 1445-1462.

[18] P. W. Randles, L. D. Libersky, Boundary conditions for a dual particle method, Computers and Structures 83 (2005) 1476-1486. 
[19] M. B. Liu, G. R. Liu, Smoothed Particle Hydrodynamics (SPH): an overview and recent developments., Archives of Computational Methods in Engineering 17 (2010) 25-76.

[20] M. Liu, G. Liu, Restoring particle consistency in smoothed particle hydrodynamics, Applied Numerical Mathematics 56 (2006) 19 - 36.

[21] J. J. Monaghan, SPH without a tensile instability, Journal of Computational Physics 159 (2000) 290-311.

[22] A. de Vaucorbeil, V. P. Nguyen, C. R. Hutchinson, A Total Lagrangian Material Point Method for solid mechanics problems involving large deformations, Computer Methods in Applied Mechanics and Engineering 360 (2020) 112783.

[23] A. de Vaucorbeil, C. R. Hutchinson, A new Total Lagrangian Smooth Particle Hydrodynamics approximation for the simulation of damage and fracture of ductile materials, International Journal for Numerical Methods in Engineering 121 (2020) 2227-2245.

[24] G. Greto, S. Kulasegaram, An efficient and stabilised SPH method for large strain metal plastic deformations, Computional Particle Mechanics 7 (2020) 523-539.

[25] T. Bode, C. WeiBenfels, P. Wriggers, Mixed peridynamic formulations for compressible and incompressible finite deformations, Computational Mechanics 65 (2020) 1365-1376.

[26] J. Bonet, A. J. Burton, A simple average nodal pressure tetrahedral element for incompressible and nearly incompressible dynamic explicit applications, Communications in Numerical Methods in Engineering 14 (1998) 437-449.

[27] C. R. Dohrmann, M. W. Heinstein, J. Jung, S. W. Key, W. R. Witkowski, Node-based uniform strain elements for three-node triangular and four-node tetrahedral meshes, International Journal for Numerical Methods in Engineering 47 (2000) 1549-1568.

[28] M. W. Gee, C. R. Dohrmann, S. W. Key, W. A. Wall, A uniform nodal strain tetrahedron with isochoric stabilization, International Journal for Numerical Methods in Engineering 78 (2009) 429-443.

[29] S. Silling, Reformulation of elasticity theory for discontinuities and long-range forces, Journal of the Mechanics and Physics of Solids 48 (2000) 175 - 209.

[30] T. Bode, C. WeiBenfels, P. Wriggers, Peridynamic Petrov Galerkin method: A generalization of the peridynamic theory of correspondence materials, Computer Methods in Applied Mechanics and Engineering 358 (2020) 112636.

[31] A. Javili, R. Morasata, E. Oterkus, S. Oterkus, Peridynamics review, Mathematics and Mechanics of Solids 24 (2019) 3714-3739.

[32] E. Madenci, E. Oterkus, Peridynamic Theory and its Applications, Springer, New York, 2014.

[33] R. C. Barta, G. M. Zhang, Analysis of adiabatic shear bands in elasto-thermo-viscoplastic materials by modified smoothed-particlen hydrodynamics (MSPH) method, Journal of Computational Physics 201 (2004) 172-190. 
[34] Z. Zhang, H. Qiang, W. Gao, Coupling of smoothed particle hydrodynamics and finite element method for impact dynamics simulation, Engineering Structures 2011 (2011) 255264.

[35] H. Hu, F. Fetzer, P. Berger, P. Eberhard, Simulation of laser welding using advanced particle methods, GAMM-Mitt 39 (2016) 149-169.

[36] H. Hu, P. Eberhard, Thermomechanically coupled conduction mode laser welding simulations using smoothed particle hydrodynamics, Computational Particle Mechanics 4 (2017) $473-486$.

[37] M. M. Rahaman, A. Pathak, D. Roy, A thermo-visco-plastic damage model and sph simulations of plugging failure, Mechanics Of Advanced Materials And Structures 25 (2017) 1374-1382.

[38] K. A. Fraser, L. I. Kiss, L. St Georges, D. Drolet, Optimization of friction stir weld joint quality using a meshfree fully-coupled thermo-mechanics approach, Metals 8 (2018) $101-125$.

[39] K. A. Fraser, L. St Georges, L. I. Kiss, Y. Chiricota, Hybrid Thermo-Mechanical Contact Algorithm for 3D SPH-FEM MultiPhysics Simulations, in: IV International Conference on Particle-Based Methods.

[40] K. Ba, A. Gakwaya, Thermomechanical Total Lagrangian SPH formulation for solid mechanics in large deformation problems, Computer Methods in Applied Mechanics and Engineering 342 (2018) 458-473.

[41] K. Damaziak, J. Malachowski, Comparison of SPH and FEM in thermomechanical coupled problems, in: AIP Conference Proceedings 2078.

[42] F. Armero, J. C. Simo, A new unconditionally stable fractional step method for nonlinear coupled thermomechanical problems, International Journal for Numerical Methods in Engineering 35 (1992) 737-766.

[43] M. Aguirre, A. J. Gil, J. Bonet, C. H. Lee, An upwind vertex centred finite volume solver for Lagrangian solid dynamics, Journal of Computational Physics 300 (2015) 387-422.

[44] P. Betsch, M. Schiebl, Energy-momentum-entropy consistent numericalmethods for largestrain thermoelasticity relyingon the GENERIC formalism, International Journal for $\mathrm{Nu}$ merical Methods in Engineering 119 (2019) 1216-1244.

[45] C. Miehe, Entropic thermoelasticity at finite strains. aspects of the formulation and numerical implementation, Computer Methods in Applied Mechanics and Engineering 120 (1995) $243-269$.

[46] M. Franke, A. Janz, M. Schiebl, P. Betsch, An energy momentum consistent integration scheme using a polyconvexity-based framework for nonlinear thermo-elastodynamics, International Journal for Numerical Methods in Engineering 115 (2018) 549-577.

[47] J. Simo, C. Miehe, Associative coupled thermoplasticity at finite strains: Formulation, numerical analysis and implementation, Computer Methods in Applied Mechanics and Engineering 98 (1992) $41-104$. 
[48] R. Ortigosa, A. J. Gil, J. Martinez-Frutos, M. Franke, J. Bonet, A new energy-momentum time integration scheme for non-linear thermo-mechanics, Computer Methods in Applied Mechanics and Engineering (2020). Acceptedss.

[49] M. Šilhavý, The Mechanics and Thermodynamics of Continuous Media, Springer Berlin Heidelberg, 1997.

[50] J. Bonet, C. H. Lee, A. J. Gil, A. Ghavamian, A first order hyperbolic framework for large strain computational solid dynamics: Part III: Thermo-elasticity, Computer Methods in Applied Mechanics and Engineering 373 (2020) 113505.

[51] J. L. Ericksen, Introduction to the thermodynamics of solids, pub-SV, New York, revised edition, 1998.

[52] J. W. Gibbs, E. B. Wilson, Vector analysis, Yale University Press, ninth edition, 1947.

[53] R. de Boer, Vektor- und Tensorrechnung für Ingenieure, Springer-Verlag, 1982.

[54] J. Bonet, A. J. Gil, R. Ortigosa, A computational framework for polyconvex large strain elasticity, Computer Methods in Applied Mechanics and Engineering 283 (2015) 10611094.

[55] J. Bonet, A. J. Gil, R. Ortigosa, On a tensor cross product based formulation of large strain solid mechanics, International Journal of Solids and Structures 84 (2016) 49-63.

[56] C. M. Dafermos, Quasilinear hyperbolic systems with involutions, Archive for Rational Mechanics and Analysis 94 (1986) 373-389.

[57] I. A. Karim, C. H. Lee, A. J. Gil, J. Bonet, A two-step Taylor Galerkin formulation for fast dynamics, Engineering Computations 31 (2014) 366-387.

[58] J. Bonet, A. J. Gil, C. H. Lee, M. Aguirre, R. Ortigosa, A first order hyperbolic framework for large strain computational solid dynamics. Part I: Total Lagrangian isothermal elasticity, Computer Methods in Applied Mechanics and Engineering 283 (2015) 689-732.

[59] C. H. Lee, A. J. Gil, J. Bonet, Development of a cell centred upwind finite volume algorithm for a new conservation law formulation in structural dynamics, Computers and Structures 118 (2013) 13-38.

[60] O. I. Hassan, A. Ghavamian, C. H. Lee, A. J. Gil, J. Bonet, F. Auricchio, An upwind vertex centred finite volume algorithm for nearly and truly incompressible explicit fast solid dynamic applications: Total and updated lagrangian formulations, Journal of Computational Physics: X 3 (2019) 100025.

[61] J. Haider, C. H. Lee, A. J. Gil, J. Bonet, A first order hyperbolic framework for large strain computational solid dynamics: An upwind cell centred Total Lagrangian scheme, International Journal for Numerical Methods in Engineering 109 (2017) 407-456.

[62] J. Haider, C. H. Lee, A. J. Gil, A. Huerta, J. Bonet, An upwind cell centred total lagrangian finite volume algorithm for nearly incompressible explicit fast solid dynamic applications, Computer Methods in Applied Mechanics and Engineering 340 (2018) 684 - 727. 
[63] M. Aguirre, A. J. Gil, J. Bonet, A. A. Carreño, A vertex centred finite volume JamesonSchmidt-Turkel (JST) algorithm for a mixed conservation formulation in solid dynamics, Journal of Computational Physics 259 (2014) 672-699.

[64] J. Bonet, A. J. Gil, R. D. Wood, Nonlinear Solid Mechanics for Finite Element Analysis: Dynamics, Cambridge University Press, 2020. Under review.

[65] A. J. Gil, C. H. Lee, J. Bonet, R. Ortigosa, A first order hyperbolic framework for large strain computational solid dynamics. Part II: Total Lagrangian compressible, nearly incompressible and truly incompressible elasticity, Computer Methods in Applied Mechanics and Engineering 300 (2016) 146-181.

[66] M. Gurtin, E. Fried, L. Anand, The Mechanics and Thermodynamics of Continua, Cambridge University Press, 2010.

[67] K. W. Q. Low, C. H. Lee, A. J. Gil, J. Haider, J. Bonet, A parameter-free Total Lagrangian Smooth Particle Hydrodynamics algorithm applied to problems with free surfaces, Computational Particle Mechanics (2020). In Press.

[68] C. H. Lee, A. J. Gil, J. Bonet, Development of a stabilised Petrov-Galerkin formulation for conservation laws in Lagrangian fast solid dynamics, Computer Methods in Applied Mechanics and Engineering 268 (2014) 40-64.

[69] A. J. Gil, C. H. Lee, J. Bonet, M. Aguirre, A stabilised Petrov-Galerkin formulation for linear tetrahedral elements in compressible, nearly incompressible and truly incompressible fast dynamics, Computer Methods in Applied Mechanics and Engineering 276 (2014) 659690 .

[70] R. Courant, K. Friedrichs, H. Lewy, On the partial difference equations of mathematical physics, Mathematische Annalem 100 (1928) 32-74.

[71] J. Feldman, J. Bonet, Dynamic refinement and boundary contact forces in SPH with applications in fluid flow problems, International Journal of Numerical methods in Engineering 72 (2007) 295-324.

[72] H. Ren, X. Zhuang, Y. Cai, T. Rabczuk, Dual-horizon perydynamics, International Journal of Numerical methods in Engineering 108 (2016) 1451-1476.

[73] Z. Dai, H. Ren, Z. Zhuang, T. Rabczuk, Dual-Support Smoothed Particle Hydrodynamics for elastic mechanics, International Journal of Computational Methods 14 (2017) 1750039.

[74] M. Franke, A. Janz, M. Schiebl, P. Betsch, An energy momentum consistent integration scheme using a polyconvexity-based framework for nonlinear thermo-elastodynamics, International Journal for Numerical Methods in Engineering 115 (2018) 549-577.

[75] R.Ortigosa, A. J. Gil, J. M. Frutos, M. Franke, J. Bonet, A new energy-momentum time integration scheme for non-linear thermo-mechanics, Computer Methods in Applied Mechanics and Engineering (2020). In Press.

[76] D. H. Wagner, Symmetric-hyperbolic equations of motion for a hyperelastic material, Journal of Hyperbolic Differential Equations 06 (2009) 615-630. 